\title{
Single Shot Time-Resolved Terahertz Spectroscopy for Optoelectronic Materials
}

by

Aleksa Djorović

A thesis

submitted to the Victoria University of Wellington in fulfilment of the requirements for the degree of Master of Science in Physics.

Victoria University of Wellington 2018 



\begin{abstract}
Optoelectronic materials and devices, such as LEDs and solar cells, are ubiquitous in the modern, technologically driven world. Understanding the fundamental physical process in optoelectronic materials is essential for the design and development of new devices which are more efficient, cheaper, printable, as well as environmentally friendly. Two particularly important material properties for device performance are charge mobility and photoconductivity, as they increase charge separation and extraction efficiencies, and thus give specific insight into device efficiency. The best suited technique for measuring mobility and conductivity on ultrafast timescales is Terahertz spectroscopy. Terahertz spectroscopy is a noninvasive, contact-free probe of the mobility of charges in optoelectronic materials. Terahertz time-domain spectroscopy allows for the direct determination of the entire complex-valued conductivity. As a result, important optical properties such as the complex refractive index and dielectric function of a material can be measured directly. The short duration of $\mathrm{THz}$ pulses, on the order of 1 ps, also allows for time-resolved studies of the transient photoconductivity in optically-excited materials with sub-picosecond time resolution, i.e. Time-Resolved Teraherz Spectroscopy (TRTS). Traditionally, only the peak of the THz pulse signal is measured with TRTS, due to the time constraints of a two-delay experiment. This does not allow for frequency-resolved $\mathrm{THz}$ spectra. As a result, it discards a lot of the information Terahertz-TDS spectroscopy contains, as well as its advantages over other spectroscopic techniques. Frequency-resolved TRTS would allow for the calculation of transient conductivity at each pump-probe delay time and can differentiate between signals of excitons
\end{abstract}


and free charge carriers. This would allow for robust interpretations of charge mobility in novel materials. However, frequency-resolved TRTS is not practically feasible in a dual-delay configuration. We develop in this thesis a novel single-shot method based on angle-to-time mapping of a rotating probe. This method is applied to build a single-shot Terahertz-TDS spectrometer. A transmissive grating applies pulse front tilt which allows for the measurement of the entire $\mathrm{THz}$ transient (over a $5.7 \mathrm{ps}$ window) in a single laser shot on a CMOS multichannel detector, thus alleviating the need for delay stage sampling of the $\mathrm{THz}$ transient, and leading to a reduction of experimental time by several orders of magnitude. An optical pump excitation is incorporated to allow a time-resolved measurement (TRTS) of the entire terahertz time-domain spectrum, and thus frequencyresolved TRTS. We show qualitative agreement between the THz time domain spectra obtained with the single shot technique and the standard free-space electro-optic (EO) sampling with balanced photodiodes, with an order of magnitude increased signal sensitivity. A proof-of-concept single shot TRTS study of a Si semiconductor sample is also given, showing we are able to resolve the TRTS signal of the entire THz pulse in a single shot, in time. This technique allows us to obtain significantly more information than traditional TRTS methods without any compromise in experiment time. However we find that the implemented single shot technique seems to suffer at higher frequencies (above $2 \mathrm{THz}$ ), which must be addressed to confirm the viability of a full spectrum single shot TRTS experiment. Further improvements, such as tighter focussing of the $\mathrm{THz}$ radiation, must be made to both the single-shot spectrometer as well as to the optical pump, for a quantitative single shot measurement. However, the proof-of-concept results in this thesis prove frequency-resolved TRTS is viable by using the developed single-shot detection method. As such it directly allows a novel spectroscopic tool which can lead to new insights into charge mobilities in optoelectronic materials, and may encourage wider application of TRTS. 


\section{Acknowledgments}

I would like to extend great thanks to everyone who contributed to last 12 months and provided help along the way.

I would like to first thank my supervisor, Justin Hodgkiss, for the opportunity to work on an exciting, new piece of science, and for guidance and support throughout the project. Building a new setup from scratch was a massive learning experience, if not a bit daunting.

Thank you to the Raman Research Group for all of your antics, pushing the boundaries of work-life balance and providing a wonderful working atmosphere in the office.

In addition, I would like to thank everyone at the Hodgkiss Research Group for being very welcoming and helpful and always happy to answer questions or provide advice if need be. They have made the 12 months of this project that much more enjoyable. For the laser people, you have been lovely lab mates and I will miss our collective frustration towards ultrafast spectroscopy.

Luke Naylor and Brendan Darby, thank you so much for your help in proof reading the thesis, you saved me some embarrassment.

Specifically, I would like to thank Shyamal Prasad for his help in general 
lab matters, as well as use of the LabView and Matlab software associated with the project. It was a gargantuan battle between man and computer for long periods and you helped tip the balance! Karen Thorn, always a calming and helpful presence in the lab, you made some of the bad lab days bearable. To Kai Chen, for his absolute lab wizardry. Always encouraging, optimistic and helpful, you made immeasurable contribution to this project.

To Nick Monahan, your help and wisdom is present in every page of this thesis. You have helped guide me from a student with no optics experience, to an ultrafast spectroscopist (maybe..). Your feedback and direction have made this project successful, and I am extremely thankful. You were patient when I asked for the 957th time for an explanation of the same optical process, and have allowed me to work and learn by doing on my own, and establishing a scientific process. I have learnt a massive ammount.

Thank you also to Assoc. Prof. Stuart Murdoch and, specifically, Baptiste Auguié, for the feedback and input on submitted thesis, leading to many improvements in the thesis. Finally, I want to acknowledge my friends and family. To all of my friends, giving me fulfilled life full of laughter and help. To my father, Andrija and Donna, (and Jet!) thank you for your support throughout the long 12 months. To the self-proclaimed Dream Team, Marko, Jovana, Luna and Agnes, for your lifelong companionship and friendship. You have always been my favourite people in the world and I look forward to the next 60 years of friendship! To Nikola (I LOVE MAXA) Maksimović, tebra samo lagano, hvala ti, ojaaaasz. To my mother, you are my scientific inspiration and greatest role model. Your ability to calm and focus me at all times is remarkable. And to Kat, you have been amazingly patient, and have made these last 12 months remarkably special, so full of love and happiness. You suprise me every day, and fine, I will acknowledge you also helped me make some figures... Volim vas! 


\section{Contents}

1 Introduction 1

1.1 Overview ....................... 1

1.2 Terahertz spectroscopy . . . . . . . . . . . . . . . 3

1.2.1 Terahertz Radiation . . . . . . . . . . . . . . . . 3

1.2.2 Terahertz Time-Domain Spectroscopy (THz-TDS) . . 5

1.2.3 Time-resolved Terahertz Spectroscopy (TRTS) . . . . 7

1.3 Ultrafast spectroscopy single-shot methods . . . . . . . . . 9

2 Development of a Terahertz Time Domain Spectrometer 15

2.1 Introduction . . . . . . . . . . . . . . . . . . 15

2.2 System design - practical considerations . . . . . . . . . 16

2.2.1 Laser system . . . . . . . . . . . . . . 17

2.2.2 Terahertz generation . . . . . . . . . . . 17

2.2.3 Detection of the $\mathrm{THz}$ waveform . . . . . . . . . . 20

2.2.4 $\mathrm{THz}$ absorption of atmospheric water . . . . . . . . . 21

2.3 Details of the THz-TDS setup . . . . . . . . . . . . . 22

2.3.1 $\mathrm{THz}$ purge box . . . . . . . . . . . . . 24

2.3.2 THz pump arm . . . . . . . . . . . . 25

2.3 .3 Probe $a r m \ldots \ldots \ldots \ldots 25$

2.3.4 Gating probe polarisation . . . . . . . . . 26

2.4 System characterization . . . . . . . . . . . 26

2.4 .1 Finding a THz signal . . . . . . . . . . . . 26 
2.4.2 THz signal amplitude dependence on $\mathrm{ZnTe}$ relative angle . . . . . . . . . . . . . . . . 29

2.4.3 Presence of étalon effects . . . . . . . . . . . . 31

2.4.4 Effect of atmospheric water on $\mathrm{THz}$ signal $\ldots . . .32$

3 Single-shot THz-TDS 37

3.1 Introduction . . . . . . . . . . . . . 37

3.2 Determining usable time windows for single-shot detection 40

3.3 Single-shot design parameters . . . . . . . . . . . . . 42

3.4 Evidence of pulse front tilt . . . . . . . . . . . . . 48

3.5 Single shot $\mathrm{THz}$ detection $\ldots \ldots \ldots \ldots$

3.6 Comparison of single shot and multishot detection . . . . . 55

3.7 Summary . . . . . . . . . . . . . . 60

4 Single-shot Time Resolved Terahertz Spectroscopy 63

4.1 Introduction . . . . . . . . . . . . . 63

4.2 Setup parameters and practical considerations . . . . . . 64

4.3 Single-shot THz-TDS of Silicon . . . . . . . . . . . . 67

4.4 Single-shot TRTS of Silicon . . . . . . . . . . . . . . . . 71

5 Future Work and Proposed Improvements $\quad 77$

6 Conclusions 83

6.1 THz-TDS spectroscopy . . . . . . . . . . . . . . . 83

6.2 Single shot THz-TDS . . . . . . . . . . . . . . . 84

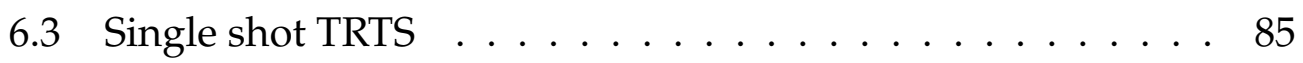




\section{Chapter 1}

\section{Introduction}

\subsection{Overview}

The ubiquity of optoelectronic materials in everyday devices has led to a dependence on the fundamental understanding of the materials and device physics. New classes of optoelectronic materials, with properties such as printability, mechanical substrate flexibility and tunability of optical properties are constantly being developed and earmarked for future optoelectronic devices[1, 2]. In addition, there is a constant driving force towards environmentally friendly materials and devices that allow lowcost fabrication methods. Tools to probe and understand the underlying working principles are required for characterization of current materials but also to guide future designs. Photo-induced charge processes play crucial roles in these materials. Understanding these fundamental physical processes is essential to the design and development of novel, and better, functional materials. However many of these processes, such as exciton formation, charge transfer and charge separation, occur on ultrafast timescales. As a result it is important to develop advanced experimental tools to monitor these properties, reveal the underlying physical mechanisms and guide design of new devices. Time-resolved ultrafast spectroscopy methods have been developed to study photo-physical proper- 
ties on sub-picosecond timescales.

Two particularly important material properties are charge mobility and photoconductivity. The best suited technique for measuring mobility and conductivity on ultrafast timescales is Terahertz spectroscopy. Terahertz spectroscopy is a non-invasive, contact-free probe of the mobility of charges in optoelectronic materials[3, 4]. The electric field is sampled directly in the time-domain which can then be transformed to the frequency domain. Frequency-resolved terahertz spectra allow the determination of the entire complex-valued conductivity, which in turn provides critical optical properties such as refractive index, the extinction coefficient and the complex dielectric function. THz pulse duration is on the order of $1 \mathrm{ps}$ allowing time-resolved studies of the transient photoconductivity in opticallyexcited materials with sub-picosecond time resolution.

Time-resolved terahertz spectroscopy (TRTS) has become a popular tool for the investigation of charge dynamics, however it can only practically monitor the time-evolution of a small number of points of the terahertz waveform. Thus, TRTS can only measure the time-dependence in the time domain. Due to the requirement of two time delays, one to sample the $\mathrm{THz}$ electric field and a second to change between the optical pump and $\mathrm{THz}$ probe, frequency-resolved TRTS is not practically feasible. The frequency spectrum is required, however, for the direct calculation of the conductivity. Time evolution of the full $\mathrm{THz}$ spectrum is desired, as it would not only allow for direct conductivity calculation, but that would in turn allow differentiation between mobility signals of excitons, polarons and free charge carriers (electrons and holes) due to their differing signatures in $\mathrm{THz}$ frequency spectra. [4, 5].

This thesis focusses on developing a method of collecting the entire $\mathrm{THz}$ waveform in a single laser shot. Application of single-shot detection to 


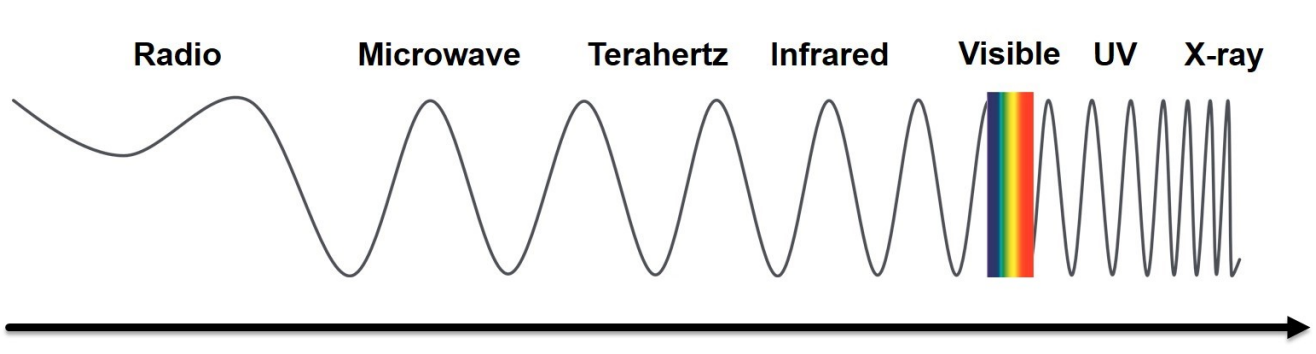

Frequency $[\mathrm{Hz}]$

Figure 1.1: A depiction of the electromagnetic spectrum, showing the position of terahertz radiation.

TRTS makes full spectral TRTS feasible, as it alleviates the need for a dual time delay. This chapter will introduce the basic principles of terahertz radiation and terahertz spectroscopy methods. The chapter will also briefly summarize and compare previously developed methods of ultrafast singleshot detection and their viability toward single-shot $\mathrm{THz}$ detection.

\subsection{Terahertz spectroscopy}

Here I briefly introduce $\mathrm{THz}$ radiation and its use in spectroscopy of the far-infrared, terahertz, region of the electromagnetic spectrum, as well as introducing the most commonly used $\mathrm{THz}$ ultrafast spectroscopy techniques and their advantages and associated issues.

\subsubsection{Terahertz Radiation}

THz radiation $\left(1 \mathrm{THz}=10^{12} \mathrm{~Hz}\right)$ most commonly refers to light in the part of the electromagnetic spectrum between 0.3 and $3 \mathrm{THz}$, corresponding to wavelengths between $100 \mu \mathrm{m}$ and $1 \mathrm{~mm}$. This positions the $\mathrm{THz}$ region between the infrared and microwave radiation regions (Figure 1.1). In the context of terahertz spectroscopy, a terahertz pulse can be described as a free-space-propagating, approximately single-cycle electromagnetic tran- 

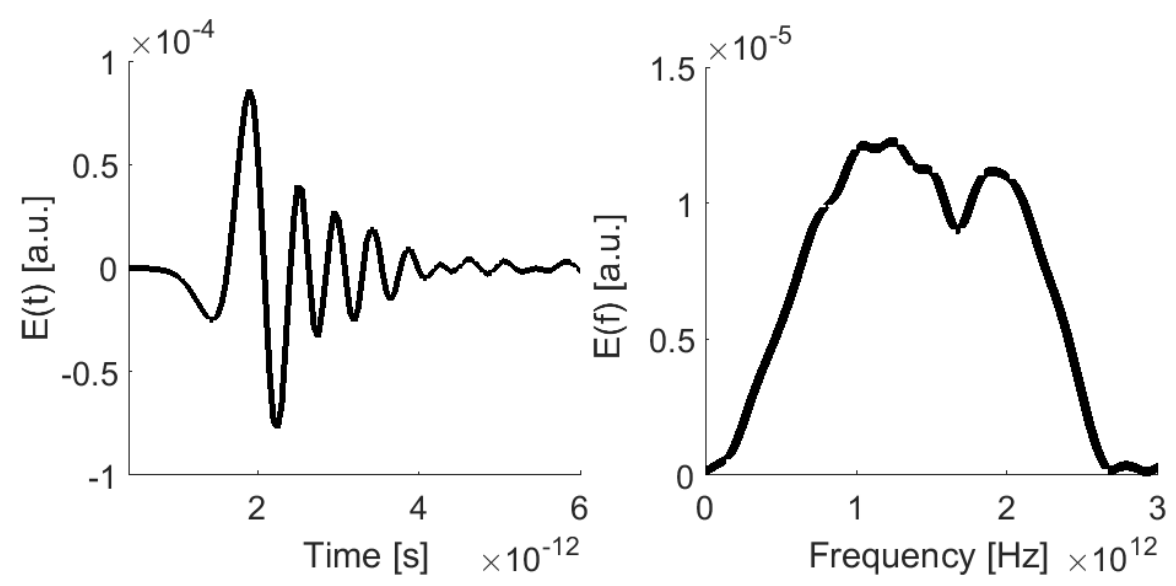

Figure 1.2: Left: A representative THz time domain spectrum. The pulse duration is $\sim 6$ ps. Right: The associated frequency spectrum obtained by Fourier transform with a bandwidth of $\sim 2.5 \mathrm{THz}$.

sient with a pulse duration on the order of 1 ps [4]. The Fourier spectrum of a terahertz pulse is often centered around $1 \mathrm{THz}$ and has a bandwidth on the order of $1 \mathrm{THz}$. A representative time domain waveform (left) and associated Fourier spectrum (right) are given in Figure 1.2. The broad spectral bandwidth allows spectroscopy on materials between 0.2-3 THz typically. The low photon energies, on the order of $\mathrm{meV}$, correspond to below room temperature thermal energies, as $k_{\mathrm{B}} T=25 \mathrm{meV}$ at room temperature, which is significantly below typical band-gap energies of $1-3 \mathrm{eV}$ in organic and inorganic semiconductors [4]. The THz region energies are summarised in Table 1.1. Typical optical phonon modes in optoelectronic materials are in the range above $5 \mathrm{THz}$ (mid-infrared), but many optically active vibrational modes appear below $5 \mathrm{THz}$ and fall into the range of $\mathrm{THz}$ spectroscopy. Therefore, $\mathrm{THz}$ spectroscopy probes low-energy excitations with resonant energies below $15 \mathrm{meV}$. Charge carriers, in particular, such as electrons, holes, excitons and polarons, all exhibit a distinct response in the low-frequency, terahertz, range of the EM spectrum $[3,4,6]$. Exciton binding energies and exciton transitions are typically in the meV range and are optically active, while the response of free carriers and polarons is 


\begin{tabular}{cccc}
$\mathrm{T}(\mathrm{K})$ & $k_{\mathrm{B}} T(\mathrm{meV})$ & $f(\mathrm{THz})$ & $\lambda(\mu \mathrm{m})$ \\
\hline 10 & 0.86 & 0.2 & 1500 \\
48 & 4.14 & 1.0 & 300 \\
96 & 8.24 & 2.0 & 150 \\
144 & 12.4 & 3.0 & 100 \\
295 & 25.4 & 6.15 & 49
\end{tabular}

Table 1.1: Conversion table between thermal energies and terahertz frequencies and wavelengths.

observed through absorption of carrier-phonon interaction modes which are in the meV energy range [3,5]. The ability to probe charge carrier intraband absorption makes $\mathrm{THz}$ spectroscopy an ideal probe of conductivity and charge mobility in optoelectronic materials.

\subsubsection{Terahertz Time-Domain Spectroscopy (THz-TDS)}

As already discussed, the terahertz, or far-infrared, region is critical to the understanding of condensed matter systems. In addition to charge carriers, the terahertz region also contains energies of inelastic scattering processes in solids as well and confinement energies in nanostructures such as quantum wells. Despite its importance, THz spectroscopy has suffered from a lack of appropriate tools. Fourier Transform InfraRed (FTIR) spectroscopy lacks the appropriate incoherent source brightness, and additionally requires the separate measurement of the real and imaginary part of the optical response functions at each frequency [7], or the use of KramersKronig transformations [8, 9]. The Kramers-Kronig relations are computationally challenging and cumbersome, and are susceptible to noisy data [10]. In addition, they require significant assumptions about the optical transitions outside the window of interest and thus rarely provide quantitative results [11]. 
Time-domain Terahertz spectroscopy represents a contact-free probe of the frequency-dependent conductivity, which is determined by mobility and carrier density. Pulsed terahertz spectroscopy has established itself as a powerful tool to study carrier dynamics in semiconductors. THz-TDS uses sub-picosend to picosecond pusles of freely propagating terahertz radiation. Sub-picosend single cycle $\mathrm{THz}$ electric field transients are generated by frequency down-conversion of femtosecond optical pulses, and are coherently detected in the time domain[3]. By measuring in the time domain, coherent detection of the transient electric terahertz field allows direct measurement of both the real (phase) and imaginary (amplitude) parts of the complex-valued refractive index of a sample. Hence, this alleviates the requirement for any Kramers-Kronig analysis[4], a significant advantage over traditional far-infrared spectroscopy techniques.

The $\mathrm{THz}$ electric field induces a change in birefringence in non-linear optical media and thus can be probed using a gating probe beam. The full electric field can be collected by sampling with a gating probe and a mechanical delay stage, i.e. by changing the time delay between the THz pump and the gating probe. The signal without the sample in place is taken as a reference followed by the measurement with the samplee. The time-domain waveforms are then transformed to the frequency domain via a Fourier transform [12]. From the frequency-resolved THz-TDS spectra, the complex refractive index $(\hat{n}=n+i k)$ can be readily extracted [4, 13]. Once the entire complex valued refractive index is known, the optical properties of the material can alternatively be expressed as the complex dielectric constant $\hat{\varepsilon}(\omega)$ or complex conductivity $\hat{\sigma}(\omega)$ using equations 1.1-1.6:

$$
\begin{gathered}
\hat{\varepsilon}=\varepsilon_{1}+i \varepsilon_{2}=\hat{n}^{2} \\
\varepsilon_{1}=n^{2}-k^{2} \\
\varepsilon_{2}=2 n k \\
\hat{\sigma}=\sigma_{1}+i \sigma_{2}
\end{gathered}
$$




$$
\begin{gathered}
\sigma_{1}=\varepsilon_{2} \varepsilon_{0} \omega \\
\sigma_{2}=-\left(\varepsilon_{1}-1\right) \varepsilon_{0} \omega
\end{gathered}
$$

where $\varepsilon_{0}$ is the permittivity of free space and $\omega=2 \pi \mathrm{f}$. The complex conductivity is the most common representation chosen in $\mathrm{THz}$ literature, as it can be used to extract the charge mobility. This is done by using appropriate models, such as the Drude model, to fit the conductivity data. In the static DC case, the conductivity is directly proportional to the charge mobility in the material, a property crtical to device performance. THzTDS is therefore an ideal spectroscopic tool for the far-infrared region of the electromagnetic spectrum. The experiment can be designed in either a transmission or reflection geometry, depending on the materials of interest.

The necessity for scanning the terahertz electric field, however, leads to several restrictions. Scanning the THz field in an unstable system can lead to hysteresis, and commonly cannot accurately measure irreversible processes[14]. Even small imperfections in the alignment of the retro-reflector on the mechanical scanning stage can lead to non-obvious effects on data quality and accuracy as the beam might move from the optimal spatial overlap position for different stage positions. Scans over large temporal ranges, or those with high time resolution (i.e. small time steps), can be time consuming and subjected to changes in the laboratory temperature and humidity over the course of the experiment.

\subsubsection{Time-resolved Terahertz Spectroscopy (TRTS)}

A particular advantage of the all-optical generation and detection of $\mathrm{THz}$ pulses in THz-TDS is the ability to combine the setup with a time-synchronized femtosecond optical excitation pulse[3]. Time-resolved terahertz spectroscopy (TRTS) is an optical pump - THz probe (OPTP) experimental technique designed to measure the excited-state transient $\mathrm{THz}$ signal. It is an exten- 
sion of the THz-TDS setup, by integrating an optical pump excitation of the sample. Using a TRTS setup, the optical pulse can be used to create charge carriers which can be monitored on the femtosecond time scale. As a result, TRTS has been used to provide insight into carrier trapping[15], cooling $[16,17]$ and recombination[16, 18], as well as the kinetics of exciton and polaron formation[5].

TRTS has been identified as a powerful tool for understanding the transient conductivity of optoelectronic materials. TRTS monitors the nonequilibrium time evolution of charge carriers and low-energy excitations. The benefit of TRTS is most notable in the differences in signals from excitons, free charges and polarons [5]. However, TRTS requires two scanning time delays, the one already present in a THz-TDS experiment, as well as a second to vary the optical pump delay compared to the THz pump. The full ability of TRTS to measure a time-dependant frequency-resolved $\mathrm{THz}$ electric field would allow for quantitative transient mobility and conductivity measurements, and robust arguments. Unfortunately, however, a full waveform TRTS is not viable as a dual-delay experiment is practically infeasible. Measurement time would span over tens of hours, which not only is impractical, but allows for sample degradation and pump bleaching, as well as shifts in laser beam pointing. All of these lead to inaccurate data. As a result, a full waveform TRTS is rarely the practice in literature. Instead, the probe scanning delay stage is set to the time -domain signal amplitude position and the optical pump time delay is varied. The TRTS dynamics from the signal peak is then used, alongside the traditional THz-TDS spectrum, to interpret the transient conductivity of the measured sample. Due to its unique ability to measure charge carrier signals, this iteration of TRTS is still extensively used, and is sufficient for observing dynamics, further highlighting its importance in the spectroscopy of condensed matter systems. 


\subsection{Ultrafast spectroscopy single-shot methods}

As discussed, most TRTS studies measure the average THz probe response, either just the amplitude peak or an integrated power, at different optical pump-probe delay times. While this method has been very useful, allowing measurement of optical pump induced carrier dynamics [16, 19], it is important to differentiate between these measurements and those in which the $\mathrm{THz}$ probe is spectrally resolved at multiple optical pump time delay points [20]. The latter experiments are rare [21, 22], as they require impractical measurement times. Spectrally resolved TRTS has the potential to deduce the extent of coupling between THz-resonant degrees of freedom [20]. As $\mathrm{THz}$ wavefroms are collected in the time domain, and require scanning of the $\mathrm{THz}$ waveform, dual delay (Optical pump to $\mathrm{THz}$ pump and $\mathrm{THz}$ pump to gating probe) $\mathrm{THz}$ experiment times scale with the number of time points (1D scan $\propto \mathrm{N}$ time points, 2D scan $\propto \mathrm{N}^{2}$ time points, etc.). As a result, fast aquisition methods are essential for their practical application. A proposed solution is to replace the traditional EO sampling, which requires the scanning of the $\mathrm{THz}$ wave, with a detection scheme that obtains the entire $\mathrm{THz}$ waveform in a single laser shot. Additionally, the $\mathrm{THz}$ waveform obtained in a single shot should, in equal signal averaging time, provide a better signal-to-noise ratio (SNR) than conventional EO sampling [20].

In this section, I will introduce a number of single-shot methods used in literature. Each method has a trade-off in terms of time window, temporal resolution, SNR and experimental complexity, and I will summarize their suitability to THz single-shot detection and implementation for TRTS. A detailed description of all of the discussed methods can be found in reference [20].

The single-shot techniques summarized can be classed as either: (I) frequency- 


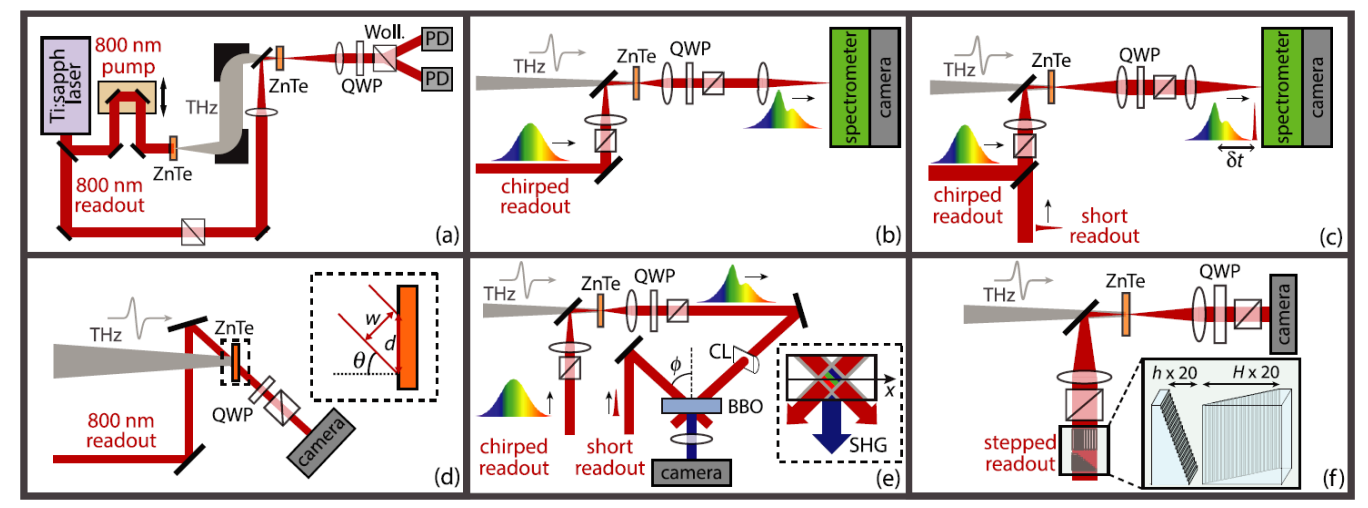

Figure 1.3: A schematic comparison of existing single-shot methods with the conventional electo-optic (EO) sampling method (a). (b): Frequency-to-time mapping by spectral encoding. (c): Temporal cross-correlation using linear spectral interferometry. (d): Space-to-time encoding with non-collinear geometry. (e): Space-to-time encoding with time domain non-collinear second harmonic crosscorrelation. (f): Angle-to-time encoding with transmissive dual echelons. Reproduced from [20].

to-time mapping, (II) space-to-time mapping and (III) angle-to-time mapping. Figure 1.3 contains a schematic of conventional electro-optic (EO) sampling (Figure 1.3(a)), compared to those of existing single-shot detection schemes.

The first frequency-to-time mapping technique is spectral encoding, depicted in Figure 1.3(b), in which a linearly chirped optical pulse is used to encode different portions of a $\mathrm{THz}$ temporal profile onto different optical frequencies in a single shot [23]. As the chirp is increased, the time window is elongated, but mapping the $\mathrm{THz}$ field onto a narrower range of frequency components degrades time resolution [20]. A 100-fs transformlimited input pulse can be chirped to a 10 ps time window, sufficient for $\mathrm{THz}$ detection, but this results in time resolution of $1 \mathrm{ps}$. As the THz pulse duration is on the order of $1 \mathrm{ps}$, this time resolution is not suitable for $\mathrm{THz}$ detection. Insufficient time resolution has been shown to lead to various 
distortions in the frequency spectrum. Despite numerous efforts in literature to overcome this serious drawback, this method and derivatives are not suitable for reliable single shot $\mathrm{THz}$ detection required for quantitative spectroscopy.

Temporal cross-correlation using linear spectral interferometry (Figure 1.3(c)) is a technique which uses both a chirped readout pulse and a short readout pulse for linear spectral interferometry. First one of the probe arms is chirped to $\sim 10 \mathrm{ps}$ in duration and is then recombined collinearly with the unchirped pulse. The THZ beam and recombined probe beams are focussed into the EO detection crystal in a way in which the THz signal is encoded onto the chirped probe pulse as either a polarization rotation or phase modulation (or combination of the two). Extraction of the THz temporal waveform is possible given a reference interferogram in the absence of a THz pulse. The temporal resolution of the method is set by the pulse width of the unchirped probe pulse, while the time window is given by the pulse width of the chirped readout pulse. As a result, this method can provide a $10 \mathrm{ps}$ time window with $100 \mathrm{fs}$ temporal resolution, making it a valid method for $\mathrm{THz}$ single-shot detection [24]. However, as for any very sensitive interferometric measurement, the experimental implementation is complex and requires strict experimental conditions.

Figure 1.3(d) shows a space-to-time encoding method using a non-collinear geometry. In this method, the readout probe samples the $\mathrm{THz}$ field when they spatially overlap in the detection crystal at different times. The mapping between the spatial coordinate and the time coordinate is calculated geometrically and depends only on the non-linear crossing angle $\theta$. As a result, the time window $\mathrm{T}$ is determined as $\mathrm{T}=w \tan \theta / c$, where $w$ is the beam width and $c$ is the speed of light in vacuum. While this method is easier to implement in comparison to the others discussed, as there is minimal modification to the conventional EO sampling method, there are 
limitations to the time window, as a large beam width is required. In addition, to achieve a large crossing area, the readout and $\mathrm{THz}$ pulses are not focussed at the detection crystal significantly reducing SNR compared to other techniques. As a result, this technique is not suitable for single-shot TRTS where we require measurement of the pump-induced change in signal, which is on the order of $1 \%$ of the standard THz-TDS signal.

The final spatial-to-time mapping technique introduced here is space-totime encoding with time domain non-collinear second harmonic crosscorrelation (Figure 1.3(e)). The $\mathrm{THz}$ is here first mapped onto a chirped readout profile, however the temporal profile of the pulse is measured by crossing it with a short readout pulse in a $\beta$-Barium borate (BBO) crystal to produce a second-harmonic signal. Different frequencies of the chirped pulse, and thus different parts of $\mathrm{THz}$ waveform, are projected onto different spatial locations on the $\mathrm{BBO}$ crystal. The spatial profile of the secondharmonic (SH) signal is recorded on a CCD array which is represented as an intensity cross-correlation of the two pulses at the detection crystal. This method does not suffer from significant time resolution degradation from the chirped pulse, however it requires a second nonlinear process, SHG, in addition to the EO effect, which significantly effects the signal intensity and SNR. Increasing the temporal window is achieved by larger beam widths which inherently lowers SHG efficiency and measurement sensitivity. As a result, the complexity of the method along with low SNR for longer time windows restrict its use for single shot $\mathrm{THz}$ spectroscopy.

Finally, angle-to-time encoding is described. In Figure 1.3(f) the method is achieved using transmissive dual echelons. This single-shot technique is built around a complementary pair of custom-made transmission-mode echelons, optics that look like glass stairways [25]. When the readout beam passes through a pair of echelons, each with $m$ steps, it splits into an array of beamlets incrementally delayed in time. The more glass the beamlet 
passes through, the longer the time delay associated with it. In the Fourier plane of a $4 f$ imaging system, the beamlets are all focussed to the same focal point at the detection crystal, but separate after the crystal and can be spatially resolved at a detector in the image plane. Modulation of the pulse train in time is mapped to modulation in intensity in the image, allowing for measurement of the entire $\mathrm{THz}$ pulse in a laser pulse. The time window is determined by the total beamlet delay time of all $m$ steps and the temporal resolution is the larger of the readout pulse duration and the time delay introduced by the smallest time increment. As a result a $10 \mathrm{ps}$ time window is easily achieved and, for practical purposes, degradation of time resolution is only noticeable for $<20$ fs transform limited readout pulses. This method has the added requirement for in-focus imaging of the probe polarization states on the camera and is thus susceptible to imaging aberrations of all of the optical components, which may affect ability to accurately measure a $\mathrm{THz}$ waveform. It, however, requires minimal modification to the probe arm in a conventional THz-TDS setup and achieves high temporal resolution and wide time windows with relative ease. As such, it presents an excellent method for $\mathrm{THz}$ detection. This is hampered by the requirement of custom-fabricated echelon optics with high optical quality.

In summary, an understanding of the trade-offs associated with current methods is essential for the development of new methods of single-shot detection, and their successful application to single shot TRTS. The two methods discussed which show viability for single-shot TRTS detection schemes are: amplitude encoding spectral interferometry (Figure 1.3(c)) and angle-to-time encoding (Figure 1.3(f)). The prior requires experimental complexity, while the latter requires custom-made optics. However, we propose a novel angle-to-time encoding single-shot method, based on the latter echelon method. The requirement for the echelons can be substituted for a diffraction grating, leading to a probe beam which is rotated about 
the focal point at the Fourier plane, allowing for tie-to-angle encoding[26]. The proposed method, developed in this thesis, retains all of the advantages of the dual echelon method with the use of readily available optics, and will be described further in chapter 3 . 


\section{Chapter 2}

\section{Development of a Terahertz Time Domain Spectrometer}

\subsection{Introduction}

The initial goal was to construct an ultrafast terahertz time-domain spectroscopy (THz-TDS) system. Terahertz spectroscopy represents a contactfree probe of the frequency-dependent conductivity, which is determined by mobility and carrier density. Pulsed terahertz spectroscopy has established itself as a powerful tool to study carrier dynamics in semiconductors. Terahertz radiation, in the energy range spanning from $1 \mathrm{meV}$ to about $100 \mathrm{meV}$, is sensitive to the response of free carriers, polarons and excitons. THz-TDS uses sub-picosecond to picosecond pulses of freely propagating terahertz radiation. Sub-picosecond single cycle $\mathrm{THz}$ electric field transients are generated by frequency down-conversion of femtosecond optical pulses, and are coherently detected in the time domain[3]. By measuring in the time domain, coherent detection of the transient electric terahertz field allows direct measurement of both the real (phase) and imaginary (amplitude) parts of the complex-valued permittivity of a sample. Hence, this alleviates the requirement for any Kramers-Kronig analysis[4], a significant advantage over traditional far-infrared spectroscopy techniques. 
The basic principle of the desired THz-TDS measurement requires a terahertz pulse inducing a change in birefringence in a birefringent material (ex. $\mathrm{ZnTe}, \mathrm{GaP}$, etc.), as well as satisfying the phase matching condition between the $\mathrm{THz}$ and femtosecond generation/detection beam, which is commonly in the near-infrared $(800 \mathrm{~nm})$. As a result, considerations must be made for:

1. Terahertz pulse generation

2. Measurement of the induced birefringence

In order to utilize this $\mathrm{THz}$ transient to characterize material properties, a measurement of the $\mathrm{THz}$ electric field without the sample in place is first performed [ $\left.E_{\text {ref }}(t)\right]$, followed by a measurement with the sample in place $[E(t)]$. The experiment can be designed in either a transmission or reflection geometry depending on the materials of interest. Another advantage of such a THz-TDS setup is the ease of combining a time-synchronized femtosecond excitation pulse, allowing for investigation of non-equilibrium carrier dynamics. It is thus also important to design the experiment with this compatibility in mind.

\subsection{System design - practical considerations}

There are multiple ways to implement a THz-TDS setup, with freedom of choice ranging from design to terahertz generation and detection methods. Here I briefly discuss the necessary practical considerations and the implications on sample choices and compatibility with a single shot spectrometer. The scope of $\mathrm{THz}$ sources and detectors is limited to those based on femtosecond laser pulses and allow for electric-field resolved measurements [3].

As the overall future goal for the experiment is to perform $\mathrm{THz}$ spectroscopy on organic photovoltaic (OPV) materials, the setup was designed 
to accommodate these samples. In the examples of $\mathrm{THz}$ spectroscopy studies of OPV materials in literature [27, 28, 29], the experiment has been designed in the transmission geometry. With this in mind, our THz-TDS spectrometer was also developed in the transmission geometry.

\subsubsection{Laser system}

The light source for our terahertz setup is a mode-locked amplified Ti:sapphire femtosecond laser system. The core of the system contains a CW pumped regenerative mode-locked Ti:sapphire oscillator (MaiTai SP, SpectraPhysics) and Ti-Sapphire regenerative amplifier (SpitfirePro, SpectraPhysics) pumped by a Q-switched Nd:YLF pump laser (Empower). The output from the Spitfire is a $\sim 100 \mathrm{fs}$ laser pulse, at a $3 \mathrm{kHz}$ repetition rate. The pulse is centred at $800 \mathrm{~nm}$ with a $\sim 10 \mathrm{~nm}$ bandwidth. The pulse width fundamentally limits the time resolution of the system, with the $\sim 100 \mathrm{fs}$ pulses allowing sub-picosecond resolution. The high repetition rate can drastically improve data quality and save on measurement time by accumulation of a large number of shots. As the fundamental $800 \mathrm{~nm}$ is a good choice for the $\mathrm{THz}$ generation beam, it is also the simplest choice for the gate probe beam.

\subsubsection{Terahertz generation}

The two most common techniques for $\mathrm{THz}$ pulse generation are photoconductivity methods and nonlinear optical processes, such as optical rectification. For the purposes of this thesis, only optical rectification will be introduced as it was the only method used in the work. For an optical rectification $\mathrm{THz}$ source, a femtosecond laser pulse passes through a second-order non-linear optical crystal (ex. ZnTe) and produces a polarization transient inside the crystal which follows the envelope of the laser pulse[4]. A simplified schematic is depicted in Figure 2.1. Optical rectification is a nonresonant second-order nonlinear process in which the de- 
velopped polarization is DC or low-frequency in nature. It can be understood as difference-frequency generation between the frequency components within the band of an optical excitation pulse[3]. A brief theoretical introduction is given here, and begins with the electric polarization of a material, which is proportional to the applied electric field $E$ and the electric susceptibility $\chi(E)$. The nonlinear optical properties of a material are described by expanding $\chi(E)$ in powers of the field:

$$
P=\left(\chi_{1}+\chi_{2} E+\chi_{3} E^{2}+\chi_{4} E^{4}+\ldots\right) E
$$

Optical rectification and the linear electrooptic effect, which is used for $\mathrm{THz}$ detection, are second order nonlinear optical effects $P_{2}^{n l}$ and are described by the $P_{2}^{n l}=\chi_{2} E^{2}$ term in the expansion. Generation of THZradiation pulses from optical rectification of fs laser pulses is based on difference frequency mixing of all frequencies within the bandwidth $\Delta \omega$ of a fs near infrared laser pulse. The frequency mixing condition comes from the interaction of two optical fields oscillating at frequencies $E_{1}=$ $E_{0} \cos \left(\omega_{1} t\right)$ and $E_{2}=E_{0} \cos \left(\omega_{2} t\right)$.

$$
P_{2}^{n l}=\chi_{2} E_{1} E_{2}=\chi_{2} \frac{E_{0}^{2}}{2}\left[\cos \left(\omega_{1}-\omega_{2}\right) t+\cos \left(\omega_{1}+\omega_{2}\right) t\right]
$$

In this case, the DC second-order nonlinear polarization $P_{2}^{n l}$ consists of a term $P_{2}^{\omega_{1}-\omega_{2}}$ proportional to the difference frequency $\omega_{1}-\omega_{2}$ which describes the production of $\mathrm{THz}$ radiation by optical rectification. There are several factors to be considered when choosing an appropriate nonlinear crystal as a THz source:

1. A large nonlinear susceptibility is required, coupled with a high damage threshold of the crystal.

2. The phase-matching condition has to be fulfilled in order to have an efficient nonlinear process. For optical rectification, this condition requires that the excitation pulse group velocity match the phase velocity of all of the generated $\mathrm{THz}$ pulse frequency components [30]. 


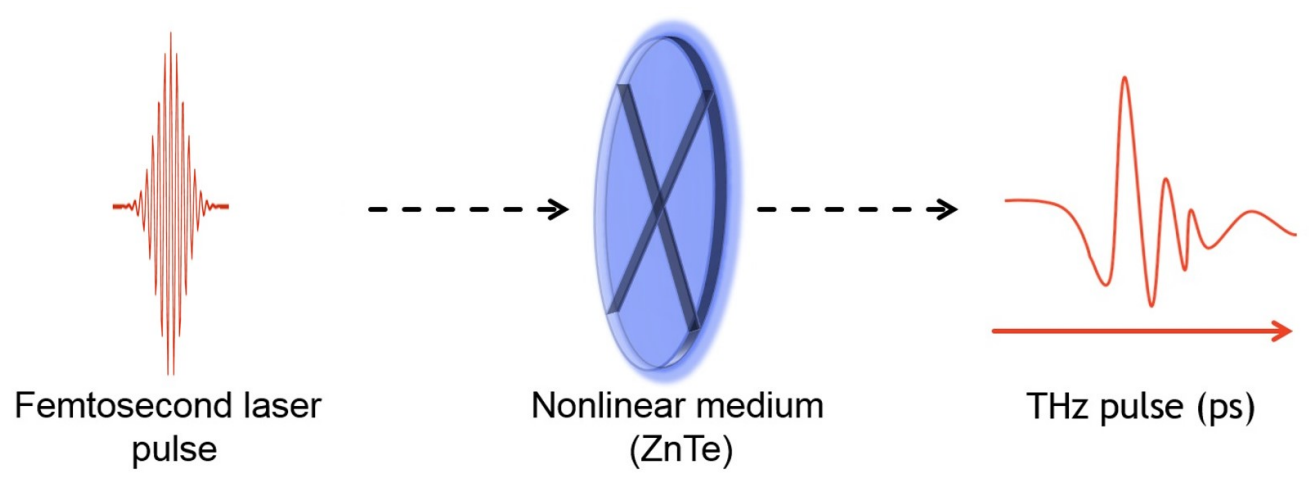

Figure 2.1: A schematic depicting the generation of a terahertz pulse by optical rectification. An optical femtosecond pulse induces a DC polarization in the nonlinear crystal (ZnTe) which leads to the emission of a terahertz radiation pulse on the order of 1 ps.

3. The material should be transparent throughout both the desired terahertz and optical frequency range. However, most commonly used inorganic crystals have phonon modes that absorb between 5 and 10 THz.

Additionally, the $\mathrm{THz}$ bandwidth is fundamentally limited by the bandwidth of the laser excitation pulse[3]. As an example, a Fourier-transform limited $100 \mathrm{fs}$ pulse at $800 \mathrm{~nm}$ is characterized by a $10 \mathrm{~nm}$ bandwidth which translates to a maximum $\mathrm{THz}$ bandwidth of about $5 \mathrm{THz}$.

With the above considerations in mind, common choices of nonlinear crystals for $\mathrm{THz}$ sources by optical rectification using a Ti:sapphire laser are ZnTe for the 0-3 THz range, GaP for 2-7 THz, and GaSe for 4-80 THz $[31,32,33]$. For our system, with a $800 \mathrm{~nm}$ fundamental with $\sim 100 \mathrm{fs}$ pulses, we chose a [110] cut $1 \mathrm{~mm}$ thick ZnTe as the THz generation crystal. 


\subsubsection{Detection of the $\mathrm{THz}$ waveform}

Just as with the $\mathrm{THz}$ generation, the detection of $\mathrm{THz}$ transients can be achieved with a nonlinear optical technique. The most widespread such method takes advantage of the linear electro-optic (EO) effect, also referred to as Pockel's effect. The EO effect produces a birefringence in materials with inversion symmetry when an electric field bias is applied. For terahertz detection, the required bias field is the electric field transient associated with the $\mathrm{THz}$ radiation. The induced birefringence causes a rotation of the polarisation of a gating probe optical beam, creating an elliptically-polarised beam which is measured by the optical power transmitted through the EO detection crystal between two crossed polarizers. To obtain the entire waveform, different points can be sampled by changing the relative pump-probe delay or by utilising various ultrafast single shot techniques to obtain the entire transient in a single laser shot (further discussed in Chapter 3). As a similar nonlinear optical mechanism is involved in both $\mathrm{THz}$ generation and detection, the same materials can be used for both processes. The polarization change is most commonly measured by free-space EO sampling with a pair of balanced photodiodes. A polarizing beam splitter, such as a Wollaston prism, is used to spatially separate the $s$ and $p$ polarizations of the probe beam and linearly polarize them. They are each then guided to one of the photodiodes. The photodiodes are connected to a Lock-in Amplifier which measures the differential signal between the two polarizations. The transmitted probe power through an EO crystal varies with the phase shift caused by the birefringence in the system. A phase shift of $\varphi_{0}=\pi / 4$ should be introduced to achieve the greatest absolute modulation in probe beam intensity for a given THz field strength [34, 35]. In a cubic crystal such as ZnTe or GaP, this phase shift can be introduced by using a quarter wave plate placed between the detection crystal and the Wollaston prism. For the designed spectrometer in this work, a ZnTe crystal ([110] and $1 \mathrm{~mm}$ thick) identical to the generation crystal was also use for $\mathrm{THz}$ detection. 


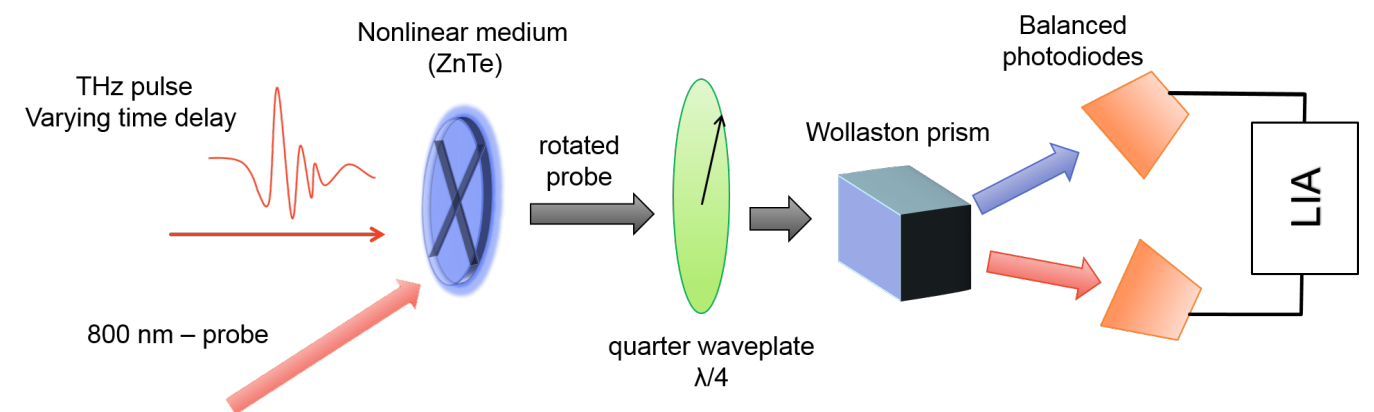

Figure 2.2: A schematic depicting the free-space electro-optic sampling detection of a terahertz pulse. The $\mathrm{THz}$ pulse induces a change in birefringence of the nonlinear medium (ZnTe in this case) which leads to a rotation of the $800 \mathrm{~nm}$ probe beam. The signal is modulated by a quarter wave-plate and the $s$ and $p$ polarizations are separated by a Wollaston prism and incident on two balanced photodiodes connected to a Lock-in amplifier where the difference signal, i.e. the electric field, is measured.

\subsubsection{THz absorption of atmospheric water}

A primary obstacle to $\mathrm{THz}$ applications is the attenuation by the ambient atmosphere due mainly to intense rotational transitions of the water molecules [36, 37]. In gases like water vapor the relative isolation of the molecules leads to sharp resonant peaks of absorption, which can dominate a THz spectrum[38]. In addition, the water medium "chirps" the terahertz pulse leading to long lived oscillations (on the order of $\sim 10 \mathrm{ps}$ ). To reduce the absorption of the $\mathrm{THz}$ radiation by water vapour during its free-space propagation between generation and detection, the spectrometer is often enclosed in a case purged with nitrogen gas or dry air. 


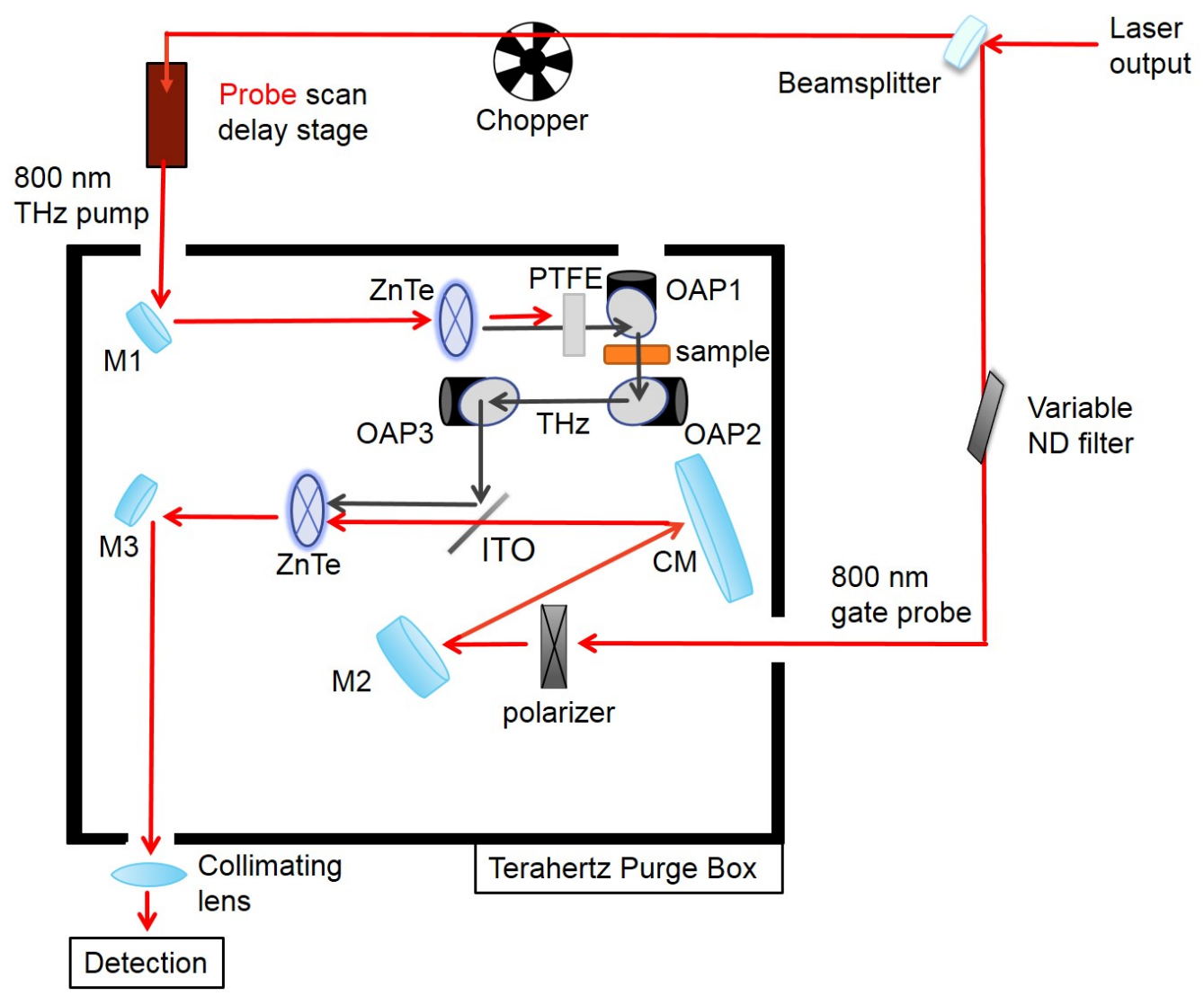

Figure 2.3: A schematic of the THz-TDS setup developed. The outside of the purge box is significantly simplified. The THz pump pathlength is varied by a mechanical delay stage, allowing the scanning of the THz probe. Separate focussing elements are used for the $\mathrm{THz}$ radiation and the $800 \mathrm{~nm}$ probe and the two arms are reconnected at a piece of Indium tin oxide (ITO) coated glass placed at $45^{\circ}$ to the optical axes. The rotated probe is collimated and detected by free-space EO sampling using a $\frac{\lambda}{4}$ waveplate, a Wollaston prism and two balanced photodiodes connected to a Lock-In Amplifier, as depicted in Figure 2.2.

\subsection{Details of the THz-TDS setup}

A schematic of the developed experimental setup is shown in figure 2.3. A Thorlabs ultrafast broadband beamsplitter - UFBS8020 - (80:20 trans- 
missive) is used to separate the $800 \mathrm{~nm}$ fundamental into two arms, the stronger, higher power, arm is used for $\mathrm{THz}$ generation - the "THz pump" arm, and the other is used for the gating probe for detection - the "probe" arm. All the optical elements inside the THz purge box are included in the figure for completion, but the beam paths outside of the box are simplified and do not include any of the reflective optics used to route the beam from the ultrafast beamsplitter to the box and are given just for perspective. The sampling of the terahertz requires a varying time delay between the $\mathrm{THz}$ transient and the probe beam. While either the probe or pump beam pathlength can be varied to sample the THz field, it is common practice to "delay" the pump beam. This also allows less possibility of small effects on the probe at the image plane for the planned single-shot integration. For this reason, in our setup, the THz pump path-length is varied. This is achieved using a retro-reflector mounted on a mechanical delay stage. In the chosen configuration, separate focussing elements are used for the $\mathrm{THz}$ radiation and the $800 \mathrm{~nm}$ probe. The two arms are reconnected at a piece of Indium tin oxide (ITO) coated glass placed at $45^{\circ}$ to the optical axes. ITO is mostly transparent to optical radiation, including $800 \mathrm{~nm}$ light, but here serves as a beamsplitter as it is optimised to reflect terahertz radiation. From the ITO, the THz transient and $800 \mathrm{~nm}$ probe propagate collinearly to the $\mathrm{ZnTe}$ detection crystal. The rotated probe is then collimated by a lense outside of the purge box and detected by balanced EO detection as described in the Detection section, using a $\frac{\lambda}{4}$ waveplate, a Wollaston prism as a polarizing beam splitter and two balanced photodiodes connected to a SR830 Lock-In Amplifier from Stanford Research Systems. An optical chopper is placed in the pump arm to lock in to the THz signal. As a $800 \mathrm{~nm}$ beam is used in both generation and detection arms, all reflective optics used are either ultrafast dielectric coated, protected gold or protected silver mirrors due to their high reflectivity at $800 \mathrm{~nm}$ (listed in order of decreasing reflectivity). Here I discuss the setup-specific details of our home-built THz-TDS setup. 


\subsubsection{THz purge box}

A $40 \mathrm{~cm} \times 40 \mathrm{~cm}$ box is used to enclose all air-sensitive aspects of the experiment, crucially the entire region in which $\mathrm{THz}$ radiation propagates. 1 inch diameter windows are placed in the positions where the laser beams enter and exit the box. A total of 4 windows were made, once each for the $\mathrm{THz}$ pump and $\mathrm{THz}$ probe beam entering the box, an additional one for the probe beam exiting the box, as well as a 4th window for the later inclusion of an optical pump beam for compatibility with a TRTS experiment. The 3 window openings where the beams enter the purge box, i.e. the $\mathrm{THz}$ pump, optical pump and $800 \mathrm{~nm}$ probe entrance windows, were fitted with $1.0 \mathrm{~mm}$ thick, 1 inch diameter Infrasil (Optical fused quartz) windows purchased from Thorlabs. These windows have a flat transmission of $>90 \%$ from the UV to the mid-IR. The window through which the rotated polarisation $800 \mathrm{~nm}$ probe exits the box was however fitted with a $1.0 \mathrm{~mm}$ thick AR-coated N-BK7 Broadband Precision Window. This was fitted with the development of the single shot detection in mind. In the case of a wave front tilted probe beam with encoded $\mathrm{THz}$ information it is crucially important to reduce the amount of dispersion of transmissive optics to a minimum. Using AR-coated N-BK7 Broadband Precision windows from Thorlabs maximises beam throughput, whilst keeping an air-tight seal of the box. Using the extremely thin window is important as to minimise imaging aberrations and any displacement of the image plane (critical in single shot detection - chapter 3). The box is connected to a dry nitrogen gas source and has a pressure release valve for excess pressure. Even without sealing the purge box lid (just closing it properly), relative humidities of $<5 \%$ are readily achievable. The relative humidities are measured with a hygrometer placed inside the box connected to a computer for real-time monitoring. As the humidity inside the box will be inhomogeneous due to an active stream of nitrogen entering the box, the hygrometer is placed as far away as possible from the $\mathrm{N}_{2}$ inlet in order to give an upper estimate of the humidity. 


\subsubsection{THz pump arm}

After the $800 \mathrm{~nm}$ beam for $\mathrm{THz}$ generation is directed into the N2 purge box. A system of one gold and 2 silver off-axis parabolic (OAP) mirrors is used to propagate the generated $\mathrm{THz}$ radiation in free-space. OAPs are used to minimise abberations, and to acheive a high numerical aperture (NA). In general, the use of reflective optics is preferred in order to reduce the dispersion of transmissive optics. The first parabolic mirror $(f=$ $50 \mathrm{~mm}$ ) is positioned to focus the $\mathrm{THz}$ radiation into the sample, the second OAP with matched focal length re-collimates the THz radiation, while the final OAP of longer focal length $(f=152 \mathrm{~mm})$ focusses the $\mathrm{THz}$ beam into the ZnTe detection crystal. For the initial setup of these optics, the $800 \mathrm{~nm}$ beam was assumed to propagate along the same beam path as the $\mathrm{THz}$ it generates by optical rectification, and was used to trace the beam propagation. Letting the $800 \mathrm{~nm}$ pump beam to propagate throughout the box allows for rough alignment of the $\mathrm{THz}$ arm of the experiment, however must only be done when the detection crystal is not in place, as a focussed $800 \mathrm{~nm}$ pump beam can damage the crystal. Thus, when operating the experiment, a $10.0 \mathrm{~mm}$ PTFE block is used as a $800 \mathrm{~nm}$ beam dump. While PTFE is completely opaque to $800 \mathrm{~nm}$ light, it is mostly transparent throughout the $\mathrm{THz}$ region of interest $(0-3 \mathrm{THz})$. It is important to not here that the designed setup assumes that the $\mathrm{THz}$ radiation generated at the crystal is near-collimated (as the first OAP is focussing). The generated $\mathrm{THz}$ will in reality be diverging from the crystal[39], and the implications of this assumption will be discussed further in the thesis in chapter 5 . In the designed geometry, we expect the $\mathrm{THz}$ radiation to be focussed at the sample, as well as at the detection crystal.

\subsubsection{Probe arm}

The $800 \mathrm{~nm}$ probe is focussed onto the ZnTe detection crystal with a 3 inch diameter $(f=200 \mathrm{~mm})$ curved mirror $(\mathrm{CM})$. The use of such a physically 
large focussing optic reduces aberration from a curved mirror and allows for the use of a large diameter probe beam, something that will become a crucial consideration when working on a single shot detection spectrometer. With this in mind, the turning mirror (M2 in Figure 2.2) used inside the box on the probe path was chosen to be a 2 inch optic, instead of the more commonly used 1 inch mirrors. The bulk of the probe arm design is based on compatibility with the latter development of the single shot detection and will be discussed in detail in Chapter 3. However, it is important to note that a variable neutral-density (ND) filter is placed in the probe beam path for easy optimisation of probe power.

\subsubsection{Gating probe polarisation}

The polarization of the probe is a crucial parameter in the measurement of $\mathrm{THz}$ signals, as the efficiency of both optical rectification and electro-optic sampling depends on the relative angle between the light polarisation and the crystal axes. It is, thus, important to establish a "clean" polarisation of both the pump and the probe beam. A wire grid polariser is used to set a vertical, i.e. s-polarisation before the splitting of the pump and probe arm. To further purify the probe beam polarisation, it passes through a second, parallel, polariser which is placed inside the $\mathrm{THz}$ purge box.

\subsection{System characterization}

The home-built THz-TDS system required significant setup as well as characterization. In this section I present the workings of setting up and characterizing a working THz-TDS Spectrometer.

\subsubsection{Finding a THz signal}

The duration of a $\mathrm{THz}$ pulse is on the order of $1 \mathrm{ps}$, which amounts to $0.3 \mathrm{~mm}$ of distance travelled by light. This means that in order to find 
the temporal overlap between the $\mathrm{THz}$ pulse and the $800 \mathrm{~nm}$ probe at the ZnTe crystal their respective pathlengths from the beam splitter need to be within less than $1 \mathrm{~mm}$. Even then, the signal amplitude has a peak width on the order of $100 \mathrm{fs}$, making finding the $\mathrm{THz}$ signal further troublesome. This is all before also considering the requirement for spatial overlap, and the fact that an un-optimized setup (both ZnTe crystal rotation angles compared to the optical axis, $800 \mathrm{~nm}$ pump power, $800 \mathrm{~nm}$ probe power, etc.) will exhibit a very low $\mathrm{THz}$ signal, if at all. It is therefore obvious that initially finding a $\mathrm{THz}$ signal of a home-built $\mathrm{THz}$ spectrometer is difficult.

However, some things can be done to help the process. An obvious starting point is the use of the $800 \mathrm{~nm}$ pump beam for the alignment of the $\mathrm{THz}$ arm and rough spatial overlap with the pump beam at the ZnTe detection crystal. While optimization of the setup is difficult without feedback from the system (before measuring a $\mathrm{THz}$ signal), the optimum pump power can be roughly found by increasing the power of the $800 \mathrm{~nm}$ pump beam incident on the generation crystal until photoluminescence after two-photon absorption is observed in the ZnTe crystal. Two-photon absorption in crystals is a process that requires high peak power. This high peak power is obtained from a limited amount of energy by ultrashort pulses. The photoluminescence is easily visible by eye as a green light is emitted from the ZnTe crystal. As this is a parasitic process competing with optical rectification, the power should then be lowered to just before the onset of photoluminescence.

Due to the issues in THz signal strength, temporal overlap is very difficult to achieve. For this reason, to find temporal overlap for our $\mathrm{THz}$ setup, we utilised second harmonic generation (SHG) in a $\beta-\mathrm{BaB}_{2} \mathrm{O}_{4}(\mathrm{BBO})$ crystal, a high-damage threshold nonlinear crystal commonly used for SHG. In this case we require two perpendicular beams overlapping and focussed on the BBO crystal with a variable delay. Only when the ultrashort pulses 


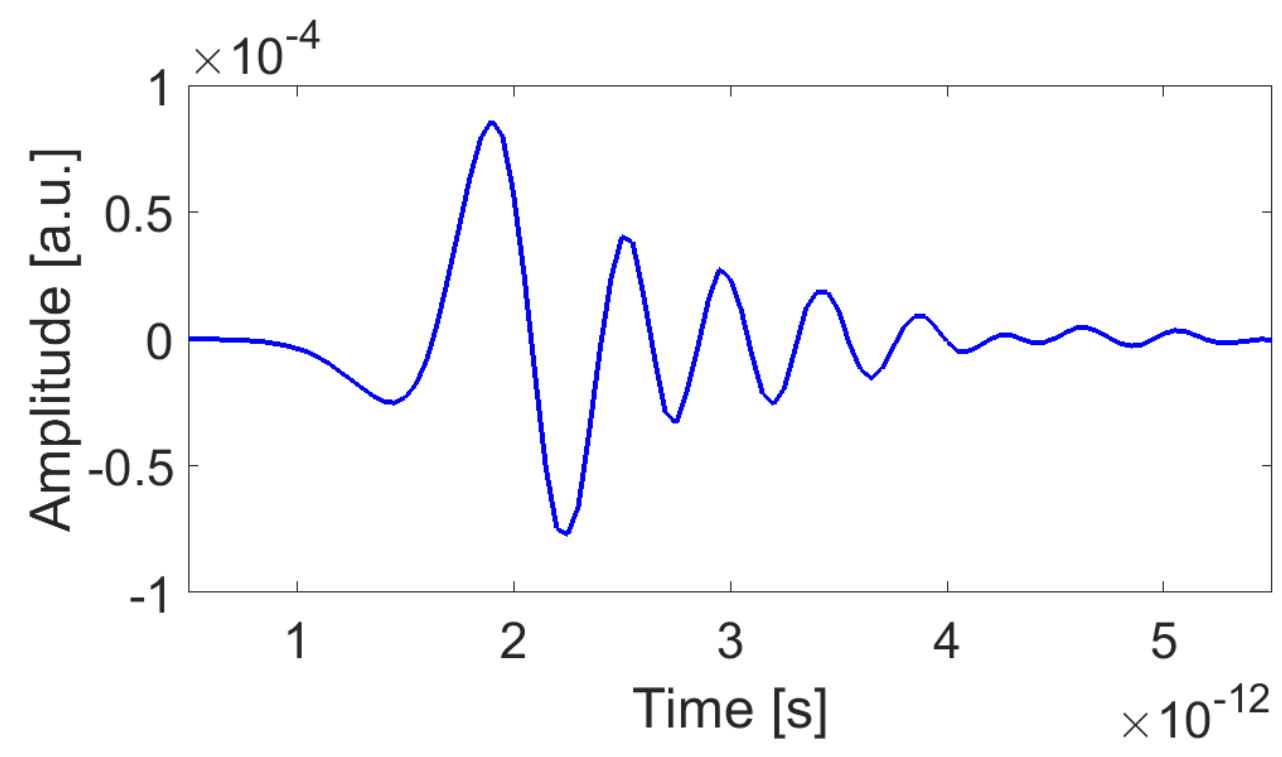

Figure 2.4: The reference $\mathrm{THz}$ waveform measured by balanced EO detection in a nitrogen atmosphere by the home-built $\mathrm{THz}$ spectrometer. The spectrum was collected over a $\sim 6$ ps range in 50 fs time steps.

overlap in time and space, will the process of SHG occur, with a $400 \mathrm{~nm}$ beam emitted at a $45^{\circ}$ angle. Thus the $800 \mathrm{~nm}$ pump beam is allowed to propagate instead of the $\mathrm{THz}$ (the PTFE block is removed) and the BBO crystal is placed in position of the ITO at $45^{\circ}$ to the pump and probe optical axes. The mechanical stage is then scanned until a time $t_{0}(\mathrm{BBO})$ is found at which SHG is visibly observed on the BBO crystal. This gives us a time delay very close to the one needed to observe the $\mathrm{THz}$ signal, and an excellent place to search for THz signal. The ITO is then returned and the spatial overlap is re-optimised. As the $\mathrm{THz}$ radiation travels through the PTFE beam block, but also the atmospheric water in the purge box with a different refractive index, some group delay is incurred leading to slight difference in time delay. However this difference is calculated to be on the order of 1-10 ps, allowing for a narrow range around the previously found $t_{0}(\mathrm{BBO})$. Utilising these methods, the $\mathrm{THz}$ signal was observed and then further optimized by pump/probe beam power, ZnTe angle, adjusted 
alignment and spatial overlap. The mechanical stage was then scanned to map the $\mathrm{THz}$ waveform in a purged $\mathrm{N}_{2}$ atmosphere $(6 \%$ relative humidity). The resulting waveform, collected over a $\sim 6$ ps range in 50 fs time steps, is given in Figure 2.4. The pump and probe intensities were optimised for maximum signal. The $800 \mathrm{~nm}$ pump beam used for $\mathrm{THz}$ generation had a beam diameter of $1.2 \mathrm{~cm}$, and had a measured power of $220 \mathrm{~mW}$ after the optical chopper. This corresponds to a generation fluence of 100 $\mu \mathrm{J} / \mathrm{cm}^{2} /$ pulse. The probe beam had a same, $1.2 \mathrm{~cm}$ beam diameter, with a power of $13 \mathrm{~mW}$ and a fluence of $3.5 \mu \mathrm{J} / \mathrm{cm}^{2} /$ pulse. The sensitivity of the system was determined by calculating the signal-to-noise ratio (SNR) at the peak signal position. Using a $300 \mathrm{~ms}$ time constant on the LIA, a SNR of $1.6 \times 10^{3}$ was achieved. As seen in the spectra obtained, this allows the resolution of all of the peaks in the $\mathrm{THz}$ waveform. The beam size of the $\mathrm{THz}$ at the sample was also measured. As $\mathrm{THz}$ is not visible, one cannot use a camera to measure, or even a card to estimate the beam spot size. In order to estimate the $\mathrm{THz}$ spot size, a pair of razor blades on a micrometer stage were used. As they blades are made of conducting metal, they reflect the $\mathrm{THz}$ radiation and $\mathrm{THz}$ only propagates through the separation of the two blades. The micrometer stage was moved to move one of the blades in relation to the other. The $\mathrm{THz}$ signal strength was monitored as it was apertured by the razor blades. This method gives a rough estimate of the $\mathrm{THz}$ spot size diameter of $\sim 3 \mathrm{~mm}$. The use of a multidimensional stage would have provided a more accurate estimate, however spatial constraints of the $\mathrm{THz}$ purge box did not allow it.

\subsubsection{THz signal amplitude dependence on $\mathrm{ZnTe}$ relative angle}

The efficiency of both $\mathrm{THz}$ generation by optical rectification and $\mathrm{THz}$ transient detection by free-space EO sampling depends on the angle between the ZnTe and the incoming beam. The pump and probe beams 


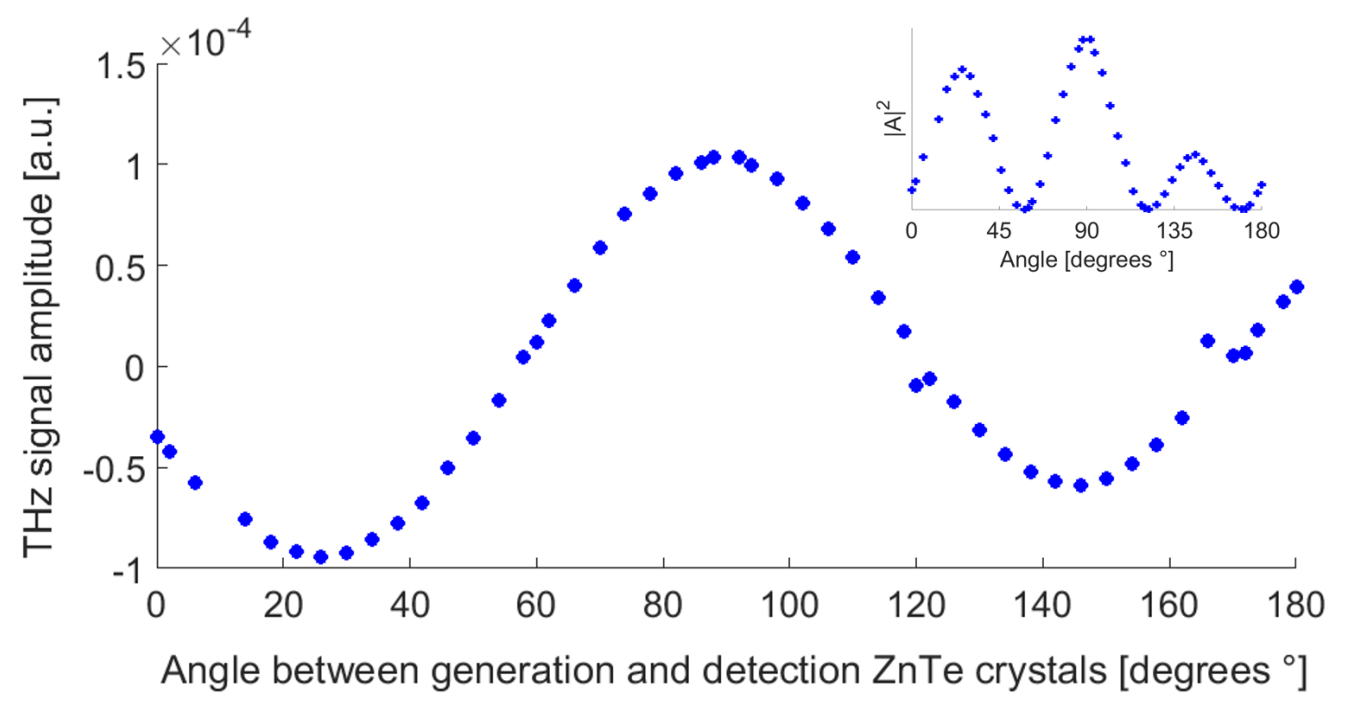

Figure 2.5: The dependence of electric field strength ( $\mathrm{THz}$ amplitude) measured by EO sampling on the relative angle between the $\mathrm{ZnTe}$ generation and $\mathrm{ZnTe}$ detection crystals. Inset: The $\mathrm{THz}$ amplitude squared is now plotted vs ZnTeZnTe angle highlighting a peak at $90^{\circ}$.

should be parallel to the [110] ZnTe face. Optical rectification depends strongly on this angle, as it controls not only the generation efficiency, but also the polarisation orientation of the $\mathrm{THz}$ field, which has consequences for the detection efficiency. The probe and $\mathrm{THz}$ field polarisations should be parallel to both each other and the [1-10] axis of the detection crystal. To investigate and optimize this, the relative angle between the two $\mathrm{ZnTe}$ crystals (generation and detection) was varied and the THz transient was collected for each angle. The signal amplitude was plotted as a function of $\mathrm{ZnTe}-\mathrm{ZnTe}$ relative angle in Figure 2.5. The figure shows that the maximum $\mathrm{THz}$ signal amplitude is detected when the relative angle between the two $\mathrm{ZnTe}$ crystals is $90^{\circ}$, or equivalently $270^{\circ}$. This is observed better in the inset of Figure 2.5 where the $\mathrm{THz}$ amplitude signal is squared. This agrees with results present in literature[40]. This geometry was locked in place and used for the remainder of the experiments. 


\subsubsection{Presence of étalon effects}

After optimization of the $\mathrm{THz}$ setup, a long range scan, extending to over $100 \mathrm{ps}$, was done to observe how long-lived the signal is, as well as to look at how large a time window is viable. The resolution of the terahertz frequency spectrum depends directly on the experimental time window of the time-domain spectrum. A longer window leads to better resolution, but extends experimental scan time, and thus finding an optimum time window to balance this trade-off is preferred. The collected long range scan is given in Figure 2.6, and spans 102.4 ps collected in 100 fs time steps. This measurement required an approximate experimental time of $55 \mathrm{~min}-$ utes.

An obvious feature in the long range scan is the appearance of multiple terahertz pulses. After the initial $\mathrm{THz}$ pulse we see attenuated waveforms equally spaced by $21.3 \mathrm{ps}$ (measured between amplitude peaks). These are identified as $\mathrm{THz}$ pulse reflections from the thick slab of PTFE. This étalon effect is commonly found in $\mathrm{THz}$ spectra of large thickness samples[13]. It is very important to note here that these pulses will add oscillation features to the Fourier spectra. Thus, the time-domain waveforms need to be time-windowed to eliminate multiple pulse reflections[4]. This fixes our maximum time window to $\sim 21$ ps for the current setup. Considering the $\mathrm{THz}$ pulse oscillations in a low-humidity atmosphere seem to cut off at $\sim 5$ ps, this is deemed acceptable. The effect of the time window length on the Fourier spectra is discussed further in Chapter 3, where this is certainly shown to be a usable time window. The étalon effects shown here proved a useful cross-check tool in further experiments due to the consistent time delay of the $\mathrm{THz}$ pulse reflections. These reflections can be eliminated by using a thinner piece of PTFE, or using a different beam block, such as highly resistive silicon. 




Figure 2.6: A long (80 ps) THz-TDS measurement of the reference waveform in low-humidity. Multiple pulse reflections are observed at a consistent spacing of $21.3 \mathrm{ps}$. These features are attributed to reflections in the thick slab of PTFE used as a $800 \mathrm{~nm}$ beam dump.

\subsubsection{Effect of atmospheric water on $\mathrm{THz}$ signal}

The effects of atmospheric water on propagating $\mathrm{THz}$ radiation are well documented in literature $[4,36,37,38]$. The rotational modes in water molecules occur in the $\mathrm{THz}$ spectral range and lead to intense absorption of several frequencies masking the $\mathrm{THz}$ spectrum. In addition to water leading to the absorption of sharp resonant peaks, terahertz electromagnetic waves experience group velocity dispersion as they propagate through a humid air medium. Thus the presence of atmospheric water leads to attenuation, as well as significant changes to the spectrum shape including prolonging the terahertz pulse. Here I discuss the effects of atmospheric water on both the time domain and frequency $\mathrm{THz}$ spectrum. 


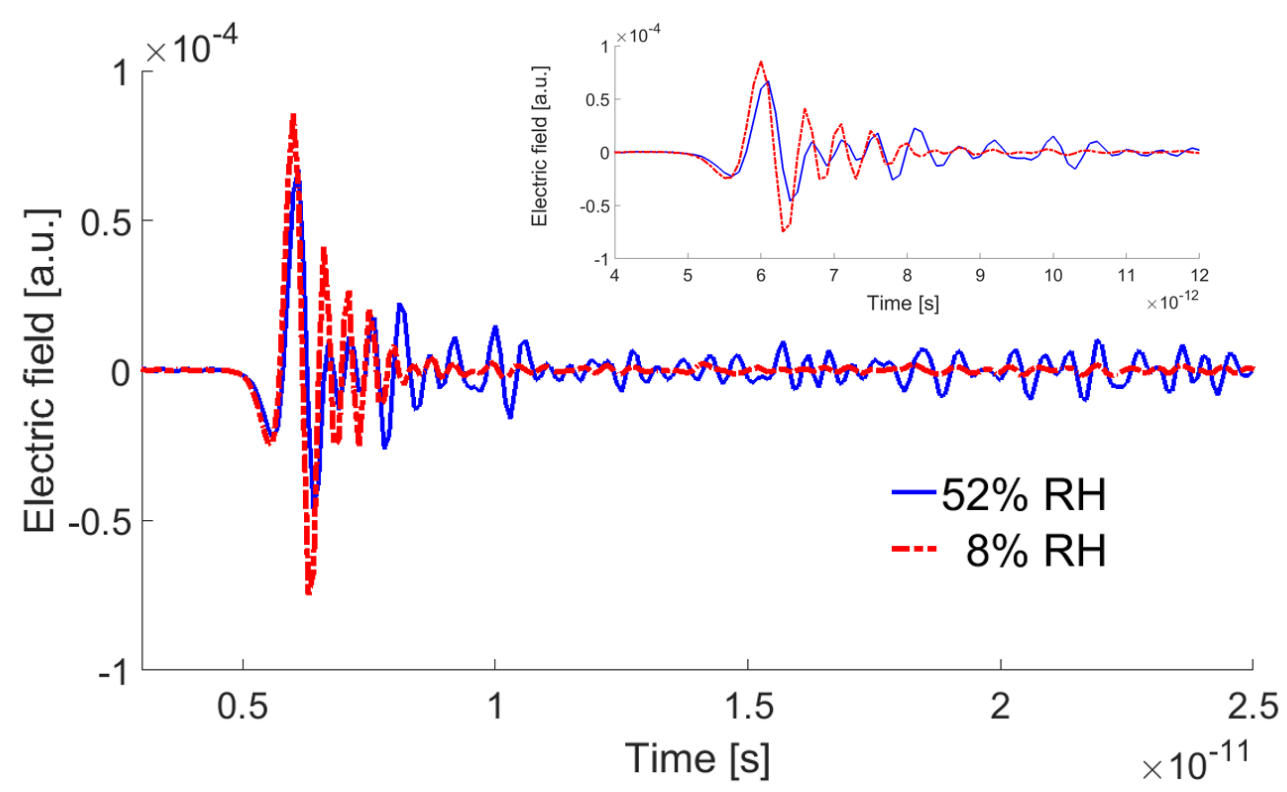

Figure 2.7: The reference $\mathrm{THz}$ waveforms measured by balanced $\mathrm{EO}$ detection in an ambient atmosphere of $52 \%$ rel. hum. (blue) and in nitrogen at $8 \%$ rel. hum. (red, dashed) over a 25 ps range in 100 fs time steps. Inset: A zoomed in plot of the region around the peak amplitude which better demonstrates the attenuation, phase shift and change in relative peak strength that occurs in ambient atmosphere.

The time-domain spectrum was collected over $\sim 25$ ps, just cutting off the first $\mathrm{THz}$ pulse reflection, both in ambient air and after purging the $\mathrm{THz}$ box with dry $\mathrm{N}_{2}$. The relative humidity of the ambient lab atmosphere (upon closing of the $\mathrm{THz}$ box) was measured to be $\sim 52 \%$. The exact same measurement was performed in a stabilised $\sim 8 \%$ relative humidity nitrogen atmosphere. The two waveforms are given in Figure 2.7. We see that while the pulse propagating through $\mathrm{N}_{2}$ has strong features only in the first $\sim 6 \mathrm{ps}$, the THz pulse which was measured in ambient air has a lot of additional dispersion imparted on it, and oscillations extend well beyond 20 ps. The inset of Figure 2.7 shows only the 8 ps range around the 
$\mathrm{THz}$ peak. We see that the atmospheric water attenuates the $\mathrm{THz}$ pulse slightly, as expected due to the absorption, and a small phase shift due to the slightly different refractive index of ambient air to a nitrogen atmosphere is also observed. Most notable though is the different relative peak strength of the two waveforms. While the $\mathrm{N}_{2}$ measurement resembles a single-cycle electric field waveform, the reflections of the pulse in the atmospheric water medium lead to "ringing" and significant asymmetry about the x-axis.

While differences in the time-domain spectrum are visible, and require a different time window to collect the full waveform, it is more important to look at the effects of water on the Fourier spectrum. The frequency spectrum is easily obtained by a Fourier transform (FT), which is given in equation 2.3 below.

$$
E(f)=\int_{-\infty}^{+\infty} E(t) \times e^{i 2 \pi f t} d t
$$

The time-domain waveforms are interpolated and are"padded" with zeros to 2048 time-points in order to get a artificially extended time window. This assumes there is no significant oscillation after the waveform, but allows for a well-behaved integral transform. The Fourier spectra obtained from the two waveforms are given in Figure 2.8. Looking at the $8 \% \mathrm{RH}$ spectrum, we generate a $\mathrm{THz}$ pulse symmetric in the frequency domain with a bandwidth of $0-2.6 \mathrm{THz}$, and peak electric field strength between 1 and $2 \mathrm{THz}$. The effect of atmospheric water is also observed and agrees with that expected from literature. We observe sharp intense absorption peaks above $1 \mathrm{THz}$, leading to significantly reduced electric field strength between $1 \mathrm{THz}$ and $3 \mathrm{THz}$. The multiple local minima coincide with water absorption lines at $0.56 \mathrm{THz}, 0.75 \mathrm{THz}, 1.16 \mathrm{THz}, 1.41 \mathrm{THz}, 1.67 \mathrm{THz}, 1.90$ $\mathrm{THz}$, and $2.26 \mathrm{THz}[14]$. We see that at $\sim 8 \%$ relative humidity we eliminate most of the water absorption peaks. A small spectral dip is still observed between 1.5 and $1.7 \mathrm{THz}$, which is well described by the intense rotational 




Figure 2.8: The Fourier THz spectra of the TDS waveforms given in Figure 2.7. The ambient air spectrum (blue) shows sharp, intense absorption peaks throughout the 1-2.5 THz region. These peaks are almost completely eradicated in the $8 \%$ rel. hum. spectrum (red, dashed). The bandwidth of the generated $\mathrm{THz}$ radiation is determined to span from $0-2.6 \mathrm{THz}$.

mode at $1.67 \mathrm{THz}\left(5260 \mathrm{~cm}^{-1}\right)[36,41]$. It is obvious that atmospheric water can mask many spectral changes in the 1-3 THz region, and therefore all subsequent experiments are done in a low-humidity nitrogen atmosphere unless specified. 



\section{Chapter 3}

\section{Single-shot THz-TDS}

\subsection{Introduction}

While THz-TDS spectroscopy has been well established as a leading tool in investigating charge mobility, the necessity for scanning the terahertz electric field has numerous issues. Scanning the $\mathrm{THz}$ field in an unstable system can lead to hysteresis, and commonly cannot accurately measure irreversible processes[14]. Even small imperfections in the alignment of the retro-reflector on the mechanical scanning stage can lead to nonobvious effects on data quality and accuracy as the beam might move from the optimal spatial overlap position for different stage positions. Scans over large temporal ranges, or those with high time resolution (i.e. small time steps), can be time consuming and subjected to changes in the lab environment, such as temperature and humidity, over the course of the experiment. Most notably, however, it restricts the measurement of timedependant transient terahertz spectra of an excited sample.

Time-resolved terahertz spectroscopy (TRTS) is a optical pump - THz probe experiment designed to measure the excited-state transient $\mathrm{THz}$ signal. It is an extension of the THz-TDS setup, by integrating an optical pump excitation of the sample. TRTS has been identified as a powerful tool for un- 
derstanding the transient conductivity of optoelectronic materials. TRTS monitors the non-equilibrium time evolution of charge carriers and lowenergy excitations. The benefit of TRTS is most notable in the differences in signals from excitons, free charges and polarons. However, TRTS requires two scanning time delays, the one already present in a THz-TDS experiment, as well as a second to vary the optical pump delay compared to the THz pump. A dual-delay experiment is practically infeasible as the TDS scan would need to be done at each optical pump - THz probe time delay. Even if a, reasonably short, 10 minute TDS scan is performed, measuring the optical pump induced time resolved dynamics at 100 time points would take an impractically long 1000 minutes, i.e. over 16 hours. If the issue of running an experiment for 16 hours itself is ignored, optical pump excitation over an extensive period of time can lead to sample degradation and the laser beam pointing itself can vary over such large experiment times, leading to inaccurate data. As a result, a full waveform TRTS is rarely the practice in literature. Instead, the probe scanning delay stage is set to the signal amplitude position and the optical pump time delay is varied only at this waveform position, as depicted in Figure 3.1. The TRTS dynamics from the signal peak is then used, alongside the traditional THz-TDS spectrum, to interpret the transient conductivity of the measured sample.

While this alleviates the issues discussed, it also discards so much of the information THz-TDS provides. One of the main advantages of THz-TDS over other far-IR spectroscopy methods is the fact that the electric field is measured directly, including both the amplitude and phase. Dynamic changes could be observed at different parts of the THz waveform, leading to new insights and interpretations. The THz-TDS waveforms at each time delay could be converted to the respective Fourier spectra, allowing for directly measured spectral kinetics across the $\mathrm{THz}$ frequency range of interest. Time dependence of the full frequency spectrum would allow for 


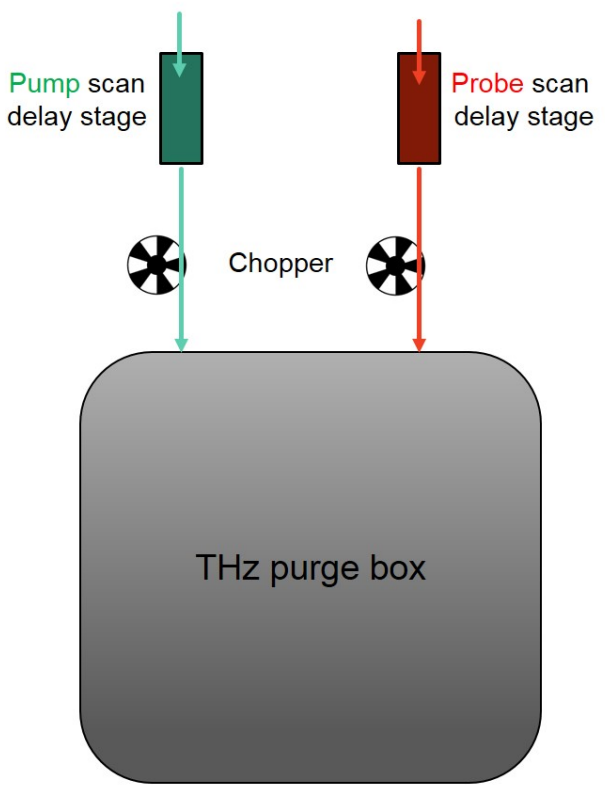

Probe scan:

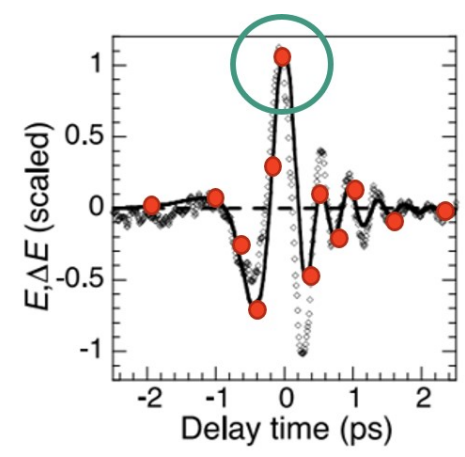

Pump scan:

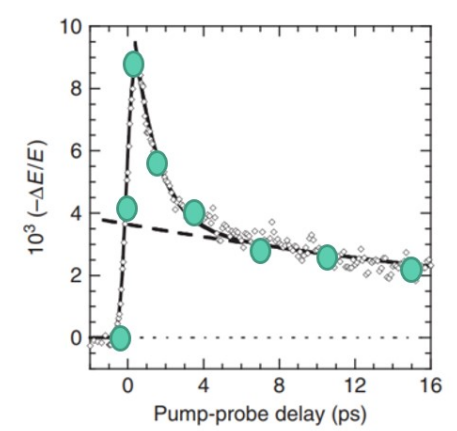

Figure 3.1: A schematic showing the method of traditional TRTS. An optical chopper is placed in the THz pump $800 \mathrm{~nm}$ beam path, and is connected to a LIA. The THz-TDS spectrum (top right, red) is then collected by a probe scan, i.e. by sampling the probe delay stage. The stage is then set to the peak amplitude position, and the optical chopper is transferred to the optical pump beam path. The pump scan delay stage is then used to obtain the TRTS pump scan (bottom right, green). ${ }^{1}$

explicit conductivity calculations and differentiation between exciton signals and those from free charges. Moreover, the optical pump excitation leads to a change in refractive index of the sample. As a result, the TDS waveform peak will shift in time slightly. The movement of the amplitude peak affects the viability of TRTS directly, as it can provide artificial kinetic features and lead to incorrectly interpreted data. All of these arguments suggest a need for a full waveform TRTS which relies on a time-efficient method to obtain the entire waveform at each optical pump delay time.

\footnotetext{
${ }^{1}$ Elements of the figure where taken from reference [27].
} 
A potential solution is to utilise methods to capture entire waveforms in a single laser shot. Single shot techniques in ultrafast spectroscopies have been used for imaging, as well as for time-efficient measurements $[26,42,43,44,45,46]$. The entire waveform can be collected by purposely chirping the $800 \mathrm{~nm}$ probe beam, using crossed beam geometries or by angle-to-time mapping, and collecting different parts of the probe on a multichannel detector. By detecting the entire waveform on a multichannel detector in a single shot, the experiment would require no additional time compared to traditional (amplitude-only) TRTS. The data can then be presented in a convenient $2 \mathrm{D}$ surface plot, as is common practice with optical ultrafast spectroscopies, such as transient absorption (TA).

In this chapter, I discuss the necessary parameters for a successful $\mathrm{THz}$ single shot experiment, the novel developed single shot detection method, the design used and the application to the already developed in-house THz-TDS spectrometer.

\subsection{Determining usable time windows for single- shot detection}

In order to collect the "entire" waveform in a single laser shot, we must determine what constitutes the beginning and end of the waveform and where it is reasonable to cut off the time window without influencing the features in the Fourier spectrum. Knowing the required time window can help then design the single shot experiment in the most effective and efficient way.

The effective single shot time window required was investigated by taking a $>20$ ps time domain measurement and truncating it at different times. 

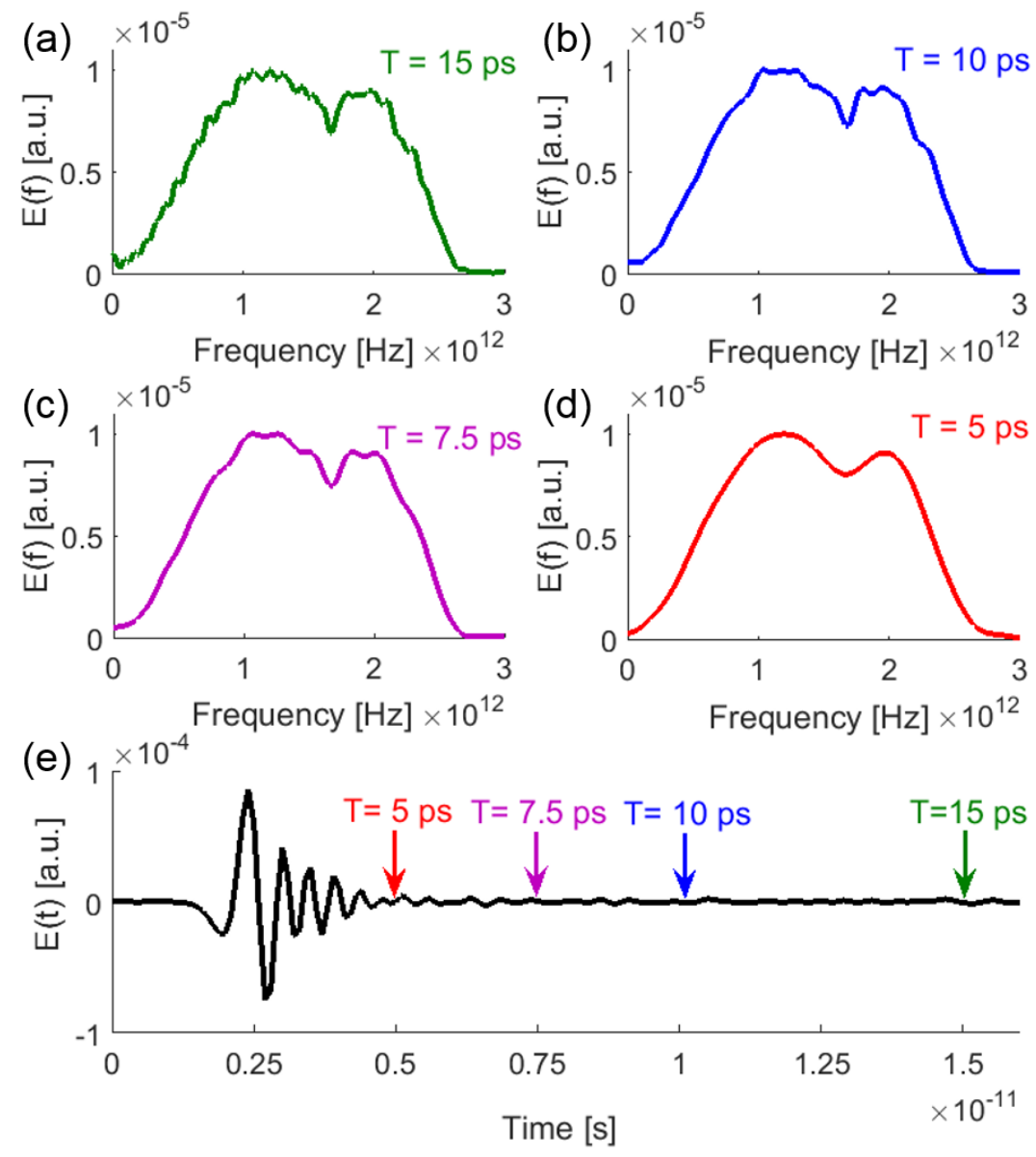

Figure 3.2: A 15 ps time domain waveform (e) is truncated to give a variety of time windows which are then transformed to the respective frequency spectra (ad). The long time window spectrum (a; green) shows high frequency resolution, while the 5 ps time window spectrum (d; red) shows no resolved features other than the major dip at $1.7 \mathrm{THz}$. The $10 \mathrm{ps}$ (b; blue) and $7.5 \mathrm{ps}$ (c; violet) spectra show no significant loss of resolution compared to (a) and are seemingly valid time windows for single shot detection.

This gave 4 different time windows, a 15 ps, 10 ps, 7.5 ps and 5 ps time window (shown in Figure 3.2(e)). The truncated waveforms were then transformed into the respective Fourier spectra which are given in Figures 
3.2(a-d). It is straight away obvious that a reduced time window lowers the frequency resolution. There is a visibly stark qualitative difference between the 15 ps window spectrum (Fig.3.2(a)) and that obtained from a 5 ps TDS waveform (Fig.3.2(d)). The 5 ps time window spectrum fails to resolve all of the smaller spectral features and seems inept for qualitative arguments. However, there seems to be no significant loss of spectral information in the $10 \mathrm{ps}$ and $7.5 \mathrm{ps}$ time window spectra suggesting these are viable single shot time windows. As a result we determine a time window of 7.5 ps to be sufficient for measurement without loss of significant spectral information. It should be noted however, that the waveform collected in Figure 3.2(e) can be translated by at least 1.5 ps to "earlier" time, before the start of the THz signal, in order to further reduce the time window without any effect on the spectrum. Hence even a time window of 6-6.5 ps is suffice, if the pre-signal time is minimised. With this in mind however, enough data should still be preserved before the signal in order to determine a background noise floor, and for background subtraction.

If a sample leads to significant dispersion and elongation of the $\mathrm{THz}$ pulse (such as that seen in atmospheric water) and the time window is no longer suitable, single shot detection can still be used by making use of the mechanical probe stage and merging multiple windows. While the entire waveform needed is no longer strictly collected in a single time window, it still significantly reduces measurement time.

\subsection{Single-shot design parameters}

As presented in the Introduction (chapter 1), the are many methods for single shot detection which can be applied to $\mathrm{THz}$ waveforms. The single shot time scan is implemented using either spatial, spectral, or angular encoding. Chirped spectral encoding methods distort the time resolutoin, while spatial encoding with a crossed beam geometry is limited by the require- 
ment of the excitation and probing of an large area[26]. The two methods determined to show viability for single-shot TRTS detection schemes were amplitude encoding spectral interferometry and angle-to-time encoding. Due to the complexity of inerferometric techinques, angle-to-time encoding seems the most attractive technique for $\mathrm{S} / \mathrm{N}$ and fast-acquisition. The implementation requires minimal modification to the probe arm of the built conventional THz-TDS setup, allows collinear excitation and probing, and an ability to achieve sufficiently long time windows with high time resolution [20].

While it provides high quality data and is easier to implement, most angleto-time encoding methods however require customised stair-step dual echelons. As a result, a novel angle-to-time single shot technique for $\mathrm{THz}$ waveforms is developed, inspired by the dual echelon method. The method is presented here, and utilises commonly available diffraction grating to rotate the probe beam. A detailed general method of ultrafast beam rotation with diffraction gratings os described in reference [26]. The relevant details and calculations are given here, however, as well as the modifications for application to $\mathrm{THz}$ spectroscopy. Due to significant spatial constraints, a transmission grating was easier to implement and was chosen over the more commonly used reflective grating.

The method is based on the principle of rotating waves[47], where a diffracted beam continuously rotates around the back focal point of a focussing element as it travels along the $x$-axis of the diffraction grating, thereby creating angular encoding. For time-resolved measurement of the $\mathrm{THz}$ pulse, the rotated probe can serve as an angular-encoded probe sampling the pulse over the time window $T$. The method is depicted in Figure 3.3, where a $\mathrm{THz}$ pulse is incident on the ZnTe detection crystal while the probe travels through a transmission grating $\mathbf{G}$ and is then focussed into the ZnTe crystal, placed in the back focal plane, by a spherical curved mir- 


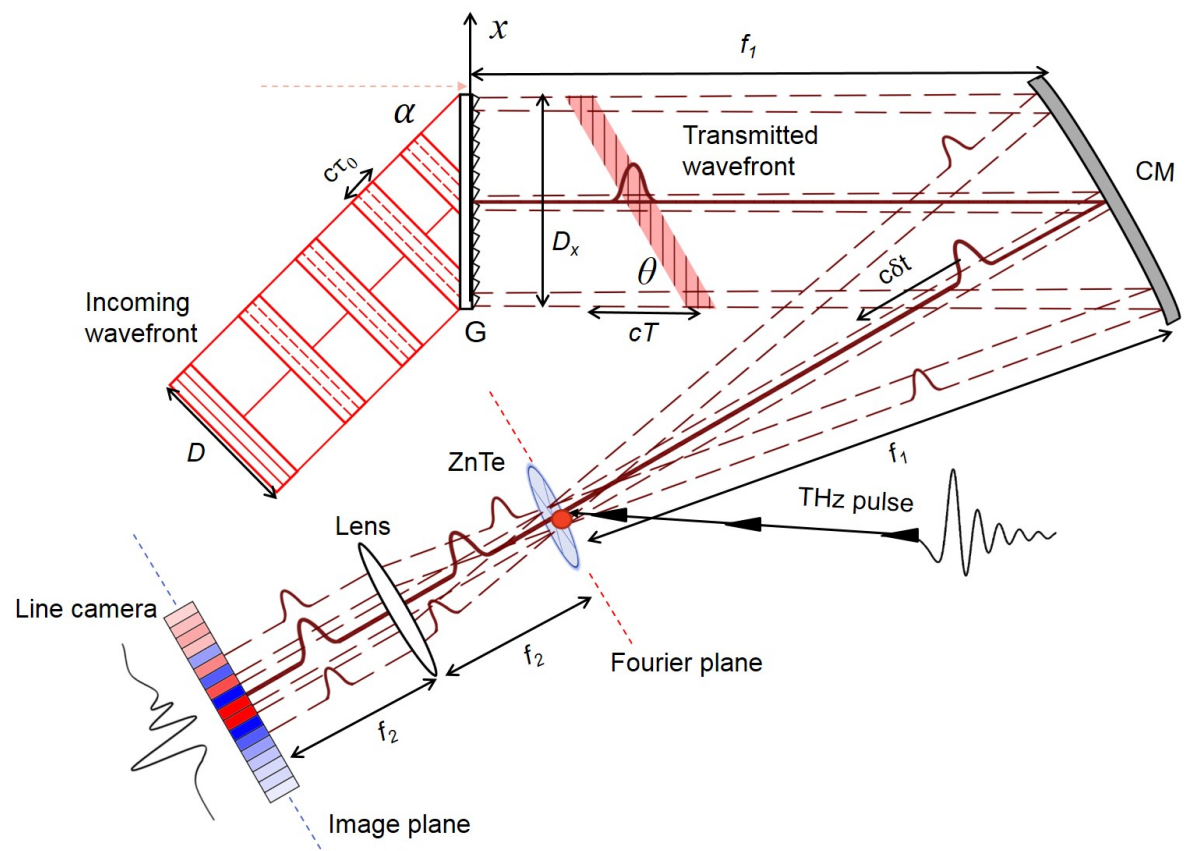

Figure 3.3: A schematic of the angle-to-time encoding of the $\mathrm{THz}$ waveform by rotation of the probe pulse around the $\mathrm{THz}$ modulated ZnTe. While only 3 beam traces are shown, the rotation is continuous around the back focal plane.The diffracted beam is parallel to the grating surface normal and the wavefront is tilted. The angular encoding is undone by a collimating lens and the probe intensity profile is measured with a line camera at the image plane. Using this method both the time window and time resolution can be adjusted to experimental needs.

ror $\mathbf{C M}$. The angular encoding is undone using a collimating lens, and the $\mathrm{THz}$ pulse is recovered at a linear detector in the image plane by the modulation of the probe intensity profile. It should be noted, however, that in the final used geometry the $\mathrm{THz}$ pulse and the focussed probe travel collinearly to the ZnTe crystal.

The gating probe beam, of diameter $D$, is made incident on the grating at an angle $\alpha$, thus the length of the illuminated part of the grating is $D_{x}=$ $D / \cos \alpha$. The transmission grating is placed in the back focal plane of the 
focussing element $(\mathbf{C M})$ and diffracts the probe pulse parallel to the surface normal (the angle of diffraction is $\beta=0$ ). As the pulse travels on the grating along the $x$-axis, the diffracted beam continuously rotates around the back focal plane (at the ZnTe crystal), creating angular encoding[26]. The pulse travels along the grating with the phase velocity $v_{x}$ :

$$
v_{c}=c / \sin \alpha
$$

At $\beta=0$, the diffraction condition is:

$$
\sin \alpha=m \lambda / b
$$

where $m$ is the diffraction order, $\lambda$ is the central wavelength of incident probe and $b$ is the grating constant. The grating imparts an angle $\theta$ between the amplitude front (light red band in Figure 3.3) and wavefront (vertical lines on the amplitude front), thus the diffracted pulse of transverse size $D_{x}$ is tilted. The beam rotates around the back focal plane as a result of this tilting. The tilt angle $\theta$ is given by:

$$
\theta=c / v_{x}=\sin \alpha
$$

Thus, the predicted experimental time window $T$ is then given by:

$$
T=D_{x} / v_{x}=D_{x} \sin \alpha / c=m \lambda D_{x} / c b
$$

Another important parameter is the pulse width at the Fourier plane, which can directly give us an estimate of the time resolution of the apparatus. Pulse broadening will occur due to angular spectral dispersion $q$ of the grating. The dispersion $q$ causes group velocity dispersion [48, 49], and at $\beta=0$ is given by:

$$
q=d \beta / d \omega=-(\sin \alpha) / \omega
$$

where $\omega$ is the center frequency of the probe pulse. The overall instrumental pulse broadening can be predicted by introducing a characteristic time of the apparatus $t_{\mathrm{ap}}$, i.e. the temporal pulse broadening due to the experimental conditions. The characteristic time depends on $q$ and the numerical 
aperture of the focussing optic NA $=\sin \left(D_{x} / f\right)$, and the time window $T$, and is given by:

$$
t_{\text {ap }}=\left(\frac{|q| T}{\sin ^{-1} \mathrm{NA}}\right)
$$

Finally, the pulse width at the Fourier plane, $\delta t$ is then given by:

$$
\delta t=\tau_{0}\left[1+\left(t_{\mathrm{ap}} / \tau_{0}\right)^{4}\right]^{1 / 2}
$$

Refer to reference [26] for a full derivation. With the above relations, an optimal choice of grating $(b)$ and optics $(q, \mathrm{NA})$ can be determined for a specific laser $\left(\tau_{0}, \omega\right)$ and specific experimental needs $(T, \delta t)$.

As the probe pulse is well defined in our setup, $\tau_{0}=100 \mathrm{fs}$ and $\omega=800$ $\mathrm{nm}$, a choice of beam size, diffraction grating and optics must be made for the wanted experimental parameters. A time window, $T$, larger than $5 \mathrm{ps}$ is needed, as well as sub-picosecond temporal resolution to resolve the $\mathrm{THz}$ pulse. A commercially available, $50 \mathrm{~mm} \times 50 \mathrm{~mm}, 300$ lines $/ \mathrm{mm}$ NIR transmission grating (Thorlabs, GTI50-03A), requires an angle of $\alpha=$ $\sin ^{-1}[m \lambda / b]=13.89^{\circ}$ for the $\mathrm{m}=1$ diffraction condition $(\beta=0)$. This is easily implemented to the probe geometry and using equation 3.4 gives an experimental time window of $T=9.1$ ps with a beam size of $\approx 11 \mathrm{~mm}$. This time window is sufficient for single shot detection, as previously determined. A $200 \mathrm{~mm}$ focal length curved mirror was chosen to focus the probe beam, determining the NA, and, using equation 3.6, the characteristic time to be $t_{\mathrm{ap}} \approx 128 \mathrm{fs}$. This corresponds to a predicted pulse width at the Fourier plane of $192 \mathrm{fs}$. This gives a maximum resolved, un-aliased, frequency of $\sim 2.6 \mathrm{THz}$ which satisfies the Nyquist - Shannon sampling theorem. This is acceptable considering the THz signal measured by balanced detection has a sharp cut off over $2.7 \mathrm{THz}$.

Considering acceptable parameters have been calculated with available optics, these were used to modify the previously built traditional $\mathrm{THz}$ spectrometer 2.3 to a single shot detection setup. A schematic of the built 


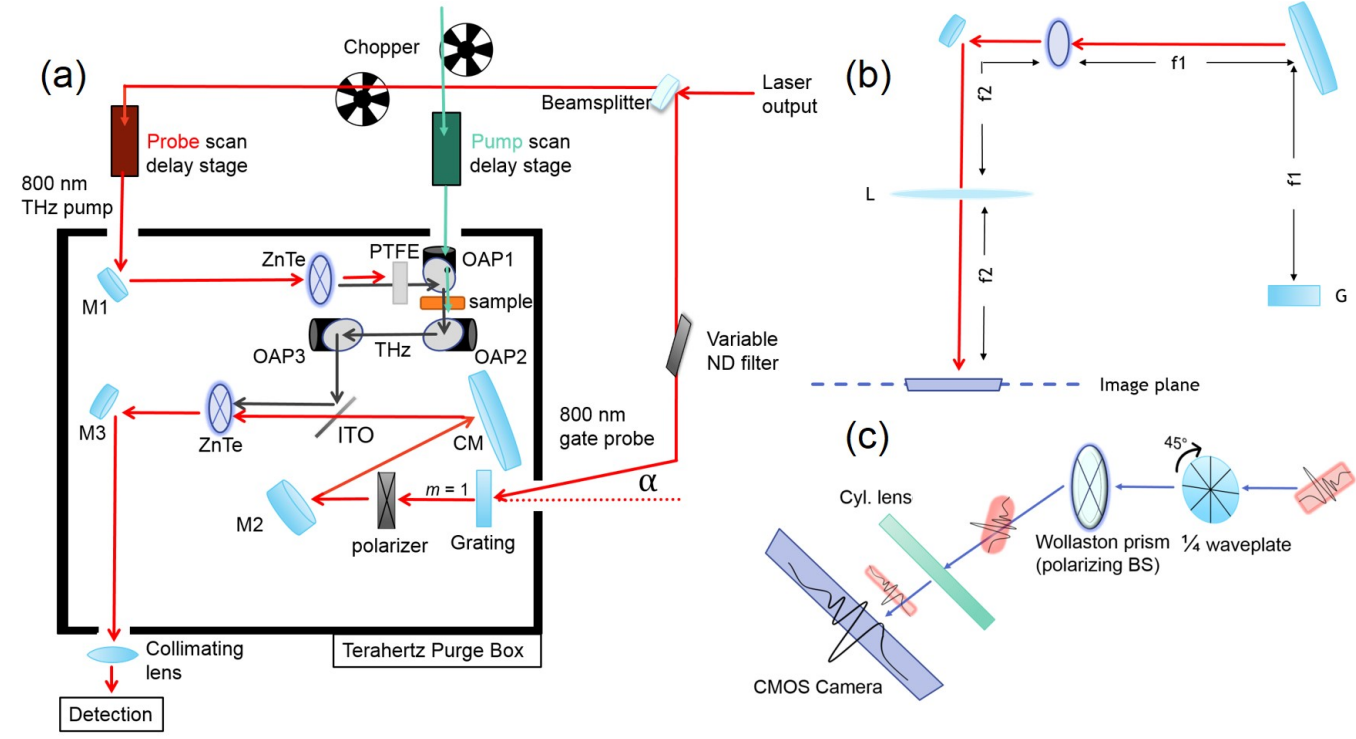

Figure 3.4: (a) A 300 lines / $\mathrm{mm}$ transmission grating is added to the standard THzTDS setup. In order to satisfy the diffraction condition for the $\mathrm{m}=1$ diffraction order, the $800 \mathrm{~nm}$ probe is made incident at an angle of $\alpha \approx 13.89^{\circ}$. (b) A representation of the $4 \mathrm{f}$ imaging system used. The grating is placed a focal length $f_{1}$ from the focussing optic, which then focusses the beam into the ZnTe detection crystal. A collimating lense is placed a focal length $f_{2}$ away from the ZnTe and the camera is finally placed at the image plane, at $f_{2}$ from the lense. (c) The tilted wavefront is detected by the use of a quarter waveplate and a Wollaston prism. The beam is cylindrically focussed onto the camera area, increasing signal intensity.

single shot spectrometer is given in Figure 3.4(a). The probe was adjusted so its incidence on the grating satisfies the $\mathrm{m}=1$ diffraction condition ( $\alpha=$ $13.89^{\circ}$ ). A LightWise Line Scan CMOS camera (LW-ELIS-1024a-1394 from Imaging Solutions Group) was placed at the image plane of a $4 \mathrm{f}$ imaging system (as shown in Fig 3.4(b)). A 2 inch diameter $f_{2}=200 \mathrm{~mm}$ uncoated collimating lense was used, matching the focussing optic in focal length $f_{1} 1: 1$, i.e. leading to a magnification power of 1 . While demagnifying the beam would be potentially beneficial in terms of fitting the entire beam profile onto the $8 \mathrm{~mm} \times 0.125 \mathrm{~mm}$ camera detector, spatial constraints, 
both from the THz box and further detection optics, required at least a 200 $\mathrm{mm}$ focal length. A cylindrical focussing lens was then used to focus the beam into a line containing all the $\mathrm{THz}$ signal encoded information onto the CMOS camera sensing area (Figure 3.4(c)). The modulation of probe intensity directly represents the electric field profile. Another noteworthy addition to the setup is the incorporated optical pump beam and "Pump" delay stage. The optical beam is incident at the sample collinearly to the $\mathrm{THz}$ by going through a small hole in the focussing OAP. The protected gold OAP has a $3 \mathrm{~mm}$ hole parallel to the focussed beam allowing for easy collinear overlap of the optical pump and $\mathrm{THz}$ pulse on the sample. This was placed in preparation for further development to a TRTS spectrometer in chapter 4.

\subsection{Evidence of pulse front tilt}

Upon setting up the desired geometry, it was necessary to find the new signal as was done when building the traditional THz-TDS spectrometer in Chapter 2. While the $\mathrm{THz}$ generation and crystal orientations were optimised, this presented a different challenge. With a large parameter space to optimize with the CMOS camera and single shot detection system, a obvious starting point is to retain the lock-in detection with the 300 lines $/ \mathrm{mm}$ transmission grating in place and find the adjusted time delay. As the probe is now tilted we expected to see an averaged signal across the time window of the wavefront tilt. The resulting measured signal is given in Figure 3.5. As expected, we observe just an averaged long lived feature. We can unambiguously diagnose this as the $\mathrm{THz}$ pulse due to the reflected pulse from the PTFE block measured at exactly the expected time delay of $\Delta t=21.3$ ps (as explained in chapter 2). The broad feature seems to be approximately $8.5 \mathrm{ps}$ long suggesting a time window of at most $\mathrm{T} \approx 8.5 \mathrm{ps}$, which is comparable to the calculated theoretical time window of $9.1 \mathrm{ps}$. The feature was used to optimize signal strength with spatial overlap and 


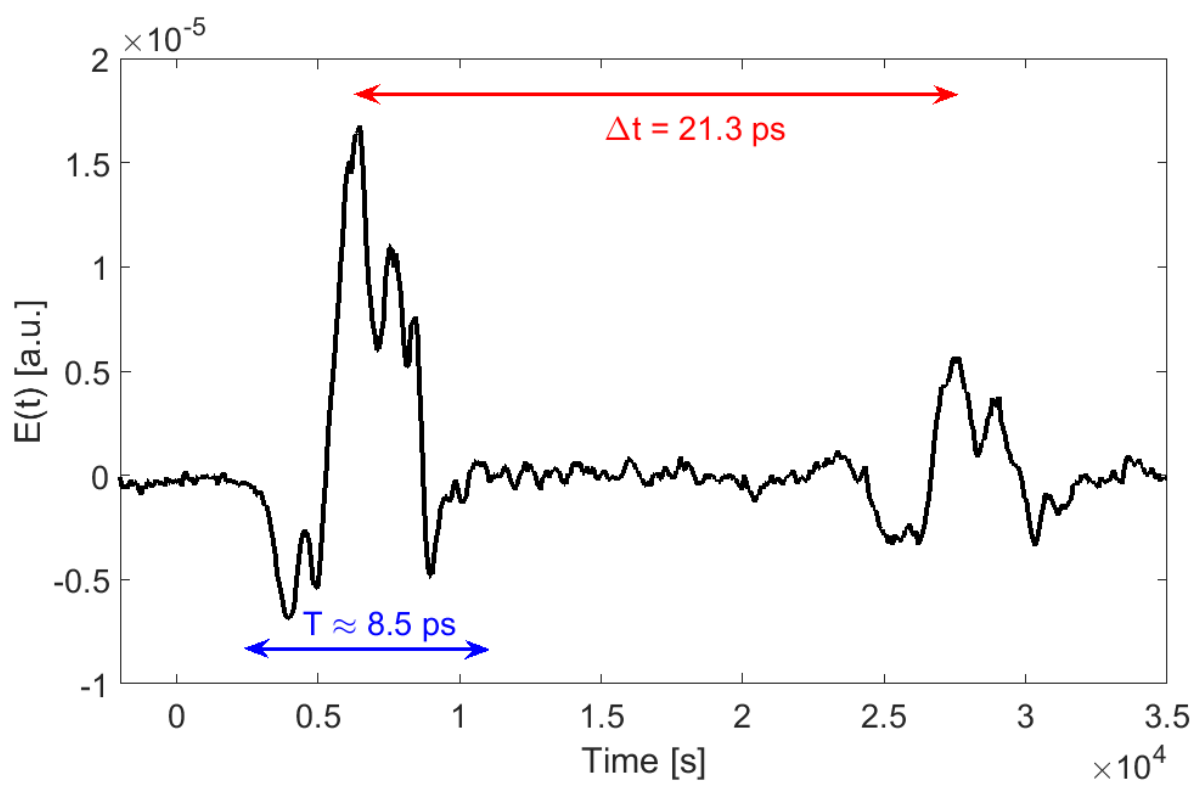

Figure 3.5: A balanced detection probe scan, over 40 ps at a time step of $100 \mathrm{fs}$, with the 300 lines $/ \mathrm{mm}$ transmission grating in place. The stretched averaged signal is evidence of pulse front tilt. The feature suggests a time window of $\sim 8.5 \mathrm{ps}$ and can be unambiguously diagnosed as the waveform signal due to a reflected pulse at exactly the expected time delay of $\Delta \mathrm{t}=21.3 \mathrm{ps}$.

probe power. The probe delay stage was set at the centre of the feature in order to allow an easier set up of the CMOS camera single shot detection.

\subsection{Single shot $\mathrm{THz}$ detection}

To detect the $\mathrm{THz}$ waveform in a single shot, the camera was now placed at the image plane of the $4 \mathrm{f}$ imaging system (shown in Fig 3.4(b)). The probe beam focussed on the ZnTe crystal and now containing angular encoding of the THz field was collimated with a 2 inch diameter $f=200 \mathrm{~mm}$ achromatic lens. A cylindrical focussing lens was then used to focus the beam into a line containing all the $\mathrm{THz}$ signal encoded information onto the $8 \mathrm{~mm} \times 0.125 \mathrm{~mm}$ CMOS line camera sensing area. 
The most straightforward, if not necessarily best, way to implement single shot detection is to set it up in the same configuration as balanced detection, with a quarter waveplate, a Wollaston prism and the CMOS camera in the position one of the photodiodes would occupy in balanced detection. Note that this means that only one polarization component is detected. The quarter waveplate is set in such a way as to minimize the transmitted light to the CMOS camera (i.e. most of the intensity in sent to the undetected polarization). This determines the signal background. When the $\mathrm{THz}$ is incident on the $\mathrm{ZnTe}$, the induced birefringence of the crystal changes the transmitted polarization which in turn increases the light intensity transmitted to the detector.

However, the background intensity was too high to directly observe any change in intensity on the detector. In order to extract the THz signal, an optical chopper was placed in the THz pump beam path, as with balanced detection, and the $800 \mathrm{~nm} \mathrm{THz}$ pump was chopped at $1500 \mathrm{~Hz}$, half the fundamental frequency of the laser output. Using pre-developed in-house Labview code the differential transmission, defined in equation 3.8, was measured in real time and allowed for detection of only changes between shots where the THz is generated and those when not.

$$
(\Delta T / T)_{\mathrm{THz}}=\frac{T_{\mathrm{THz}}^{\mathrm{ON}}-T_{\mathrm{THz}}^{\mathrm{OFF}}}{T_{\mathrm{THz}}^{\mathrm{OFF}}}
$$

By measuring the normalised differential transmission, averaged over 3000 shots, the ambient atmosphere time domain waveform in figure 3.6(black) was obtained. The $\mathrm{THz}$ probe power was optimized by the variable ND filter to $13 \mathrm{~mW}$, i.e. a fluence of $3.5 \mu \mathrm{J} / \mathrm{cm}^{2} /$ pulse. The $\mathrm{THz}$ pump power was retained at $100 \mu \mathrm{J} / \mathrm{cm}^{2} /$ pulse, as was used for traditional THz-TDS. Firstly, we can visibly observe that we have qualitatively resolved the same lineshape as in balanced detection. One of the pixels on the CMOS camera does not work and the peak at 740 pixels is an artifact due to the 
dead pixel, which will be present in all single shot spectra presented in this thesis. We also see a fall-off at the edges of the detection window. This was expected to be due to the fall off in intensity of the probe beam away from the centre. Even though the furthest parts of the beam are not incident on the detector, due to the $\sim 11 \mathrm{~mm}$ beam diameter and the smaller $8 \mathrm{~mm}$ detector width, we observe that the signal is only measurable between pixels 200 and 900 when the beam is centred. This means that 300 pixels, i.e. over a quarter of total pixels are unusable in the current geometry.

In order to reduce the effects on the edge of the window, the probe beam was magnified using a Galilean telescope built between the variable ND filter and the beam entering the THz purge box. A $f=-20 \mathrm{~mm}$ concave and a $f=60 \mathrm{~mm}$ convex lense were used to build a beam expander with a magnifying power of $M=3$. This would have led to $a \approx 33 \mathrm{~mm}$ probe beam diameter, but due to the use of a 1 inch diameter lense in the beam expander, the beam itself was apertured to 1 inch, i.e. $25.4 \mathrm{~mm}$ in diameter. For this reason, mostly 2 inch and 3 inch diameter optics were used inside the $\mathrm{THz}$ purge box, allowing for an equally large probe beam exiting the box (due to the 1:1 focal length matching of the focussing and collimating optics). Expanding the beam however reduces the fluence by a factor of $\mathrm{M}^{2}$ as the intensity $I \propto d^{2}$. To maintain the same fluence at the ZnTe crystal as used with a $11 \mathrm{~mm}$ probe beam of $3.5 \mu \mathrm{J} / \mathrm{cm}^{2} /$ pulse, the ND filter was used to increase the average power by a factor of 9 , to $115 \mathrm{~mW}$. Due to the finite size of the Wollaston prism aperture, however, the beam is effectively apertured to $10 \mathrm{~mm}$. A large beam is more likely to have a "flat" intensity profile around the centre, and it was thus expected that aperturing the beam with the Wollaston prism would allow for a flat beam profile over the $8 \mathrm{~mm}$ active area, and thus higher quality data. It is worth noting that the beam here can be then demagnified, subject to physical space and appropriate optics, to "fit" through the Wollaston prism giving a larger experimental time window. However, the same drop off at the beam edges 


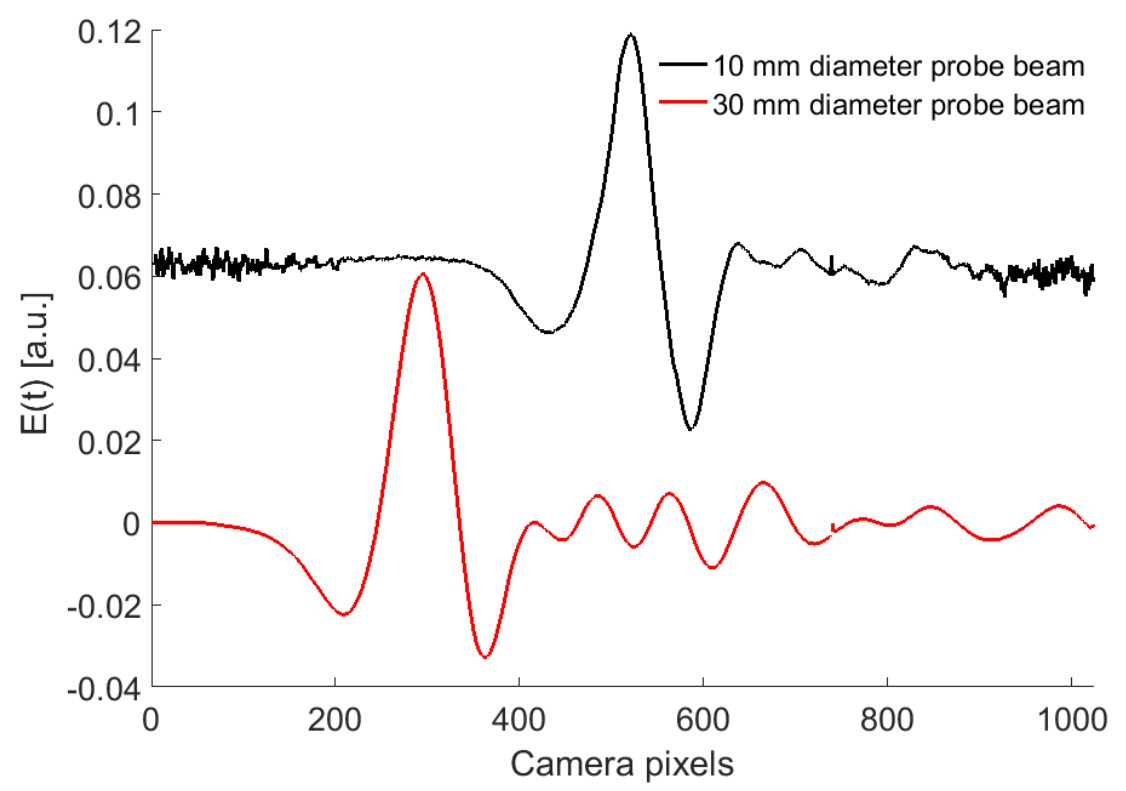

Figure 3.6: The ambient atmosphere $\mathrm{THz}$ waveforms collected with the single shot THz-TDS spectrometer with a $\sim 10 \mathrm{~mm}$ diameter (black) and a $\sim 30 \mathrm{~mm}$ diameter (red) probe beam. Due to the largely varying probe intensity across the $8 \mathrm{~mm}$ detector area, the $10 \mathrm{~mm}$ probe waveform suffers from signal variability across the pixel range. In addition, the $10 \mathrm{~mm}$ waveform shows no signal at all between approximately pixels 0-200 and 900-1024. These issues are alleviated by having a large, $\sim 30 \mathrm{~mm}$ diameter beam apertured so that the central, flat, intensity profile is incident on the $8 \mathrm{~mm}$ wide detector.

would have been observed. A $25.4 \mathrm{~mm}$ probe beam leads to an estimated experimental time window of 21 ps if the entire beam is incident. However, as the camera are is only $8 \mathrm{~mm}$ wide, this is, without demagnifying, the part of the probe beam which we measure. We can estimate the expected time window by $21 \mathrm{ps} \times(8 / 25.4)=6.61 \mathrm{ps}$. The application of the expanded beam led to the use of all 1024 pixels, as can be seen in the obtained ambient atmosphere waveform in figure 3.6 (red). This method was used in all subsequent single shot measurements. 
The nitrogen atmosphere measurement $(4 \% \mathrm{RH})$ was taken and plotted in figure 3.7(a). This measurement was used to calibrate the pixel-to-time axis transformation. The probe delay stage was used to move the waveform move across the detection window by a known time, here in $500 \mathrm{fs}$ increments. The pixel position of the signal peak was recorded and plotted as a function of the time delay in Figure 3.7(b). We see that the pixel to time mapping is linear and a calibration relation of 1 pixel $=5.59 \mathrm{fs}$ is obtained. A linear calibration gives evidence of a well collimated beam at the image plane. The pixel resolution of $5.6 \mathrm{fs}$ is not practically important as the fundamental time resolution of the single shot setup is $\sim 200 \mathrm{fs}$. However it does show that much more of the time window, or alternatively the other polarization, can be also placed on the camera without loss of time resolution. The experimental time window can now be determined as $\mathrm{T}=1024$ $\times 5.59 \mathrm{fs}=5.724 \mathrm{ps}$. The amplitude of the peak as it was moved across the detection window was also measured and plotted as a function of pixel position in 3.7(c). This shows no significant intensity dependence of signal on pixel position, further giving merit to expanding the beam and creating a flat intensity beam profile on the detector.

The method performed only captures one of the polarization components however, and thus in the least reduces the potential signal strength, but is also oblivious to potential differences in the two polarization signals. Three solutions are proposed here. The first is to use a second, synchronised camera to detect the neglected polarization. While this would give the closest comparison to balanced photodiode detection, it was practically unattainable in this thesis due to the lack of synchronised cameras. The second was to use a Wollaston prism or polarizing beam displacer to place both polarization components onto the same camera. While both the Wollaston prism and beam displacer will add a slight pathlength difference to the two polarization components, these would be minuscule and well within the range of the depth of field of the detection. This method 
(a)

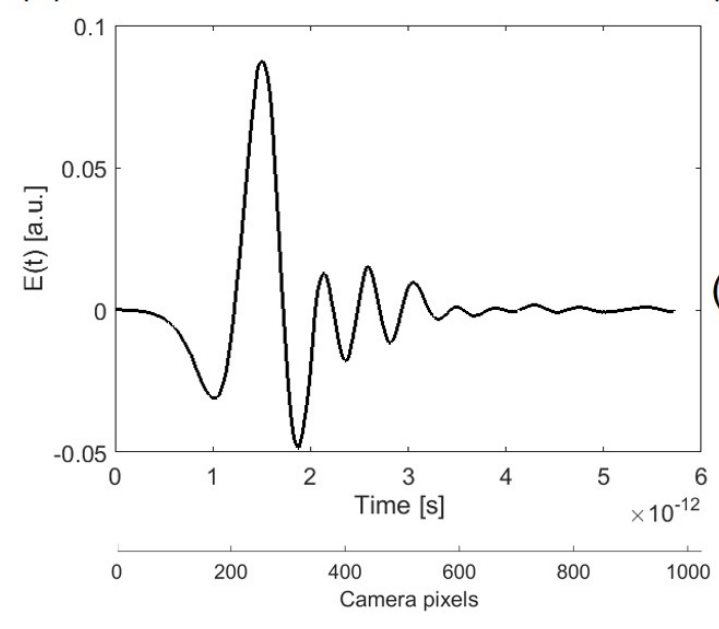

(b)

(c)
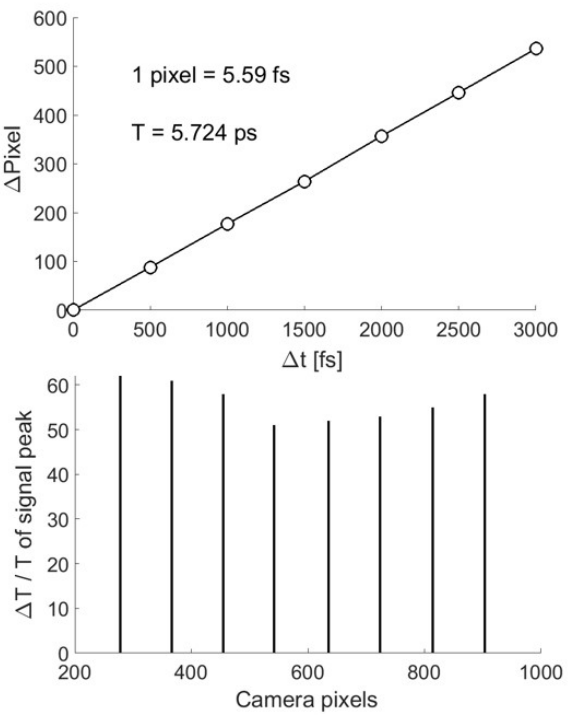

Figure 3.7: (a) The low humidity ( $4 \% \mathrm{RH}) \mathrm{N}_{2}$ atmosphere $\mathrm{THz}$ waveform collected with the single shot THz-TDS spectrometer shown as a function of both camera pixel as well as calibrated time. (b) The calibration curve mapping pixel to time delay showing a linear mapping. The conversion gives us a calculated experimental time window of 5.724 ps. (c) The peak intensity as a function of pixel position shows a relatively flat intensity distribution, giving validity to the single shot measurement.

would require fitting two waveforms, side by side (or conversely one under the other) onto the same camera sensing area. With the available CMOS camera, this would mean separating the 1024 pixels into two domains with some necessary separating pixels in-between them. Considering the physical size of the CMOS width of $8 \mathrm{~mm}$, it would be physically difficult to implement. First the two beams would need to be demagnified and then placed on the camera without overlap. This could also be alleviated with the use of a physically longer CMOS camera, or by using a 2D detector and binning two regions separately. In the interest of time constraints, this method was left as an option for future improvement. The third option was to use the crossed the crossed polarizer detection method. As the 
$800 \mathrm{~nm}$ probe has a well defined, vertical, polarization, a second polarizer can be placed after the ZnTe detection crystal in an orientation such as to minimize the transmitted light. This configuration can then be used to measure the leaked transmission upon $\mathrm{THz}$ incidence, leading to direct EO detection, without separating polarization components. While this method seems the most effective, I was unable to successfully implement it in this thesis. It is possible that the extinction of the polarizer available $\left(\approx 10^{-3}\right)$ was too low. Regardless, it remains a method that should be further investigated, as it allows a simple method of obtaining the entire $\mathrm{THz}$ signal.

\subsection{Comparison of single shot and multishot de- tection}

It is primarily important to compare the new single shot method to the traditional EO sampling method. To be a viable alternative to balanced detection for qualitative and quantitative learnings, the single shot waveform and corresponding Fourier spectrum need to be equivalent to those obtained by balanced detection. Before discussing the comparisons it is important to note that due to the nature of the single shot implementation, i.e. measuring only one polarization, no exact comparison with the balanced detection (contains both polarizations) can be made. While attempts to provide a better comparison method were made on both single and multishot sides, the crossed polarizer method did not give the expected results and measuring photodiode EO sampling detection with just one photodiode was not possible due to a high background noise. Thus all comparisons, unless specified differently, are of a single polarization single shot experiment and a full balanced photodiode detection scan.

Figure 3.8(a) gives the single shot waveform compared to the traditional 

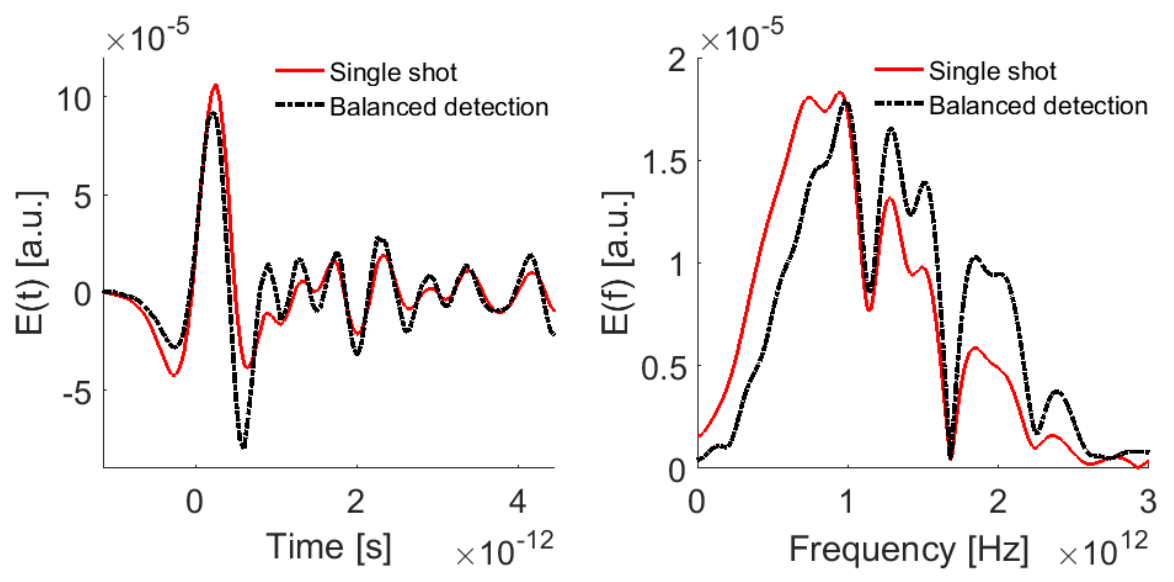

Figure 3.8: A comparison of the ambient air (45\% rel. hum.) THz pulse measured by single shot method and balanced detection in the time domain (a) and in the frequency domain (b). In the time domain we see the waveform has a difference in relative peak strength which leads to a lower intensity in the frequency spectrum above $1 \mathrm{THz}$.

TDS waveform in ambient air. It is obvious that all the same peaks and features are present, but there is a distinct difference in relative peak strengths. The respective Fourier spectra (Figure 3.8(b)) show that the single shot detection gives weaker electric field intensities at higher frequencies, above $1 \mathrm{THz}$. It also interestingly shows an earlier onset of bandwidth at low frequency. This is even more pronounced in the low humidity $\mathrm{N}_{2}$ atmosphere measurement comparison given in Figure 3.9(a). The single shot seems to bias toward the initial peak, while subsequent peaks have significantly lower relative intensities. This discrepancy is again transferred to the respective Fourier spectra for the low humidity waveforms shown in Figure 3.9(b) and is exhibited by reduced $\mathrm{THz}$ bandwidth over $\approx 2 \mathrm{THz}$. This difference can result from many issues. Initially, it was thought that it may have stemmed from measuring only a single polarization. In an attempt to determine this, the Wollaston prism was rotated $180^{\circ}$ to now measure the second polarization, and the waveplate adjusted so as to not saturate the detector. As the waveplate was adjusted in between mea- 

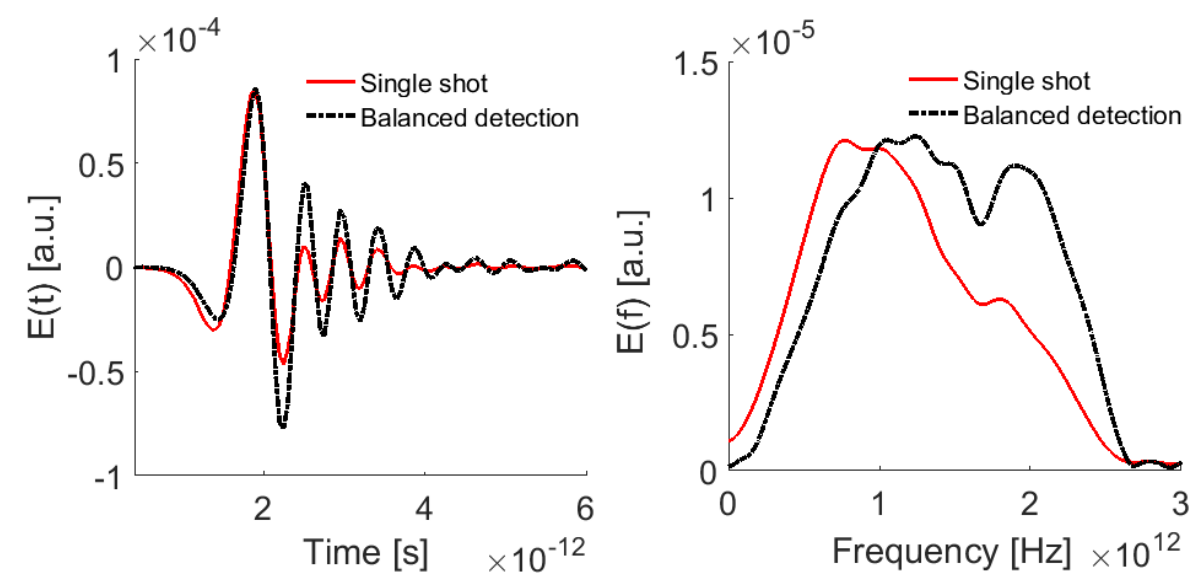

Figure 3.9: A comparison of the low humidity $(6 \%)$ nitrogen atmosphere $\mathrm{THz}$ pulse measured by single shot method and balanced detection in the time domain (a) and in the frequency domain (b). In the time domain we see a distinct drop in intensity after the initial peak and a similar sharp drop in intensity in the frequency spectrum above $1 \mathrm{THz}$ as in the ambient air measurement.

surements, the relative signal intensities of the two polarizations are not equivalent, and the full signal cannot be obtained by simply summing the two contributions as measured. However, if the discrepancy between the two detection techniques comes solely from the measurement of one vs two polarization components, then a linear combination of the two polarization components separately measured by single shot detection should exist such that it matches the balanced detection signal. The two polarizations were measured in ambient atmosphere however were only able to be both collected over an $\sim 4$ ps window. The combined signal $S_{\text {total }}$, defined in equation 3.9, was optimised by the coefficients $c_{1}$ and $c_{2}$ to give the best match to the balanced detection signal. The best agreement was obtained with a combination of $c_{1}=0.09$ and $c_{2}=0.91$. While the Fourier spectrum is of low resolution due to the small time window used, it is still however suffice to make a qualitative comparison. It is obvious that the combined signal still shows significant discrepancies in both the time domain (Figure 3.10(a)) and frequency spectra (Figure 3.10(b)). Again the single shot 

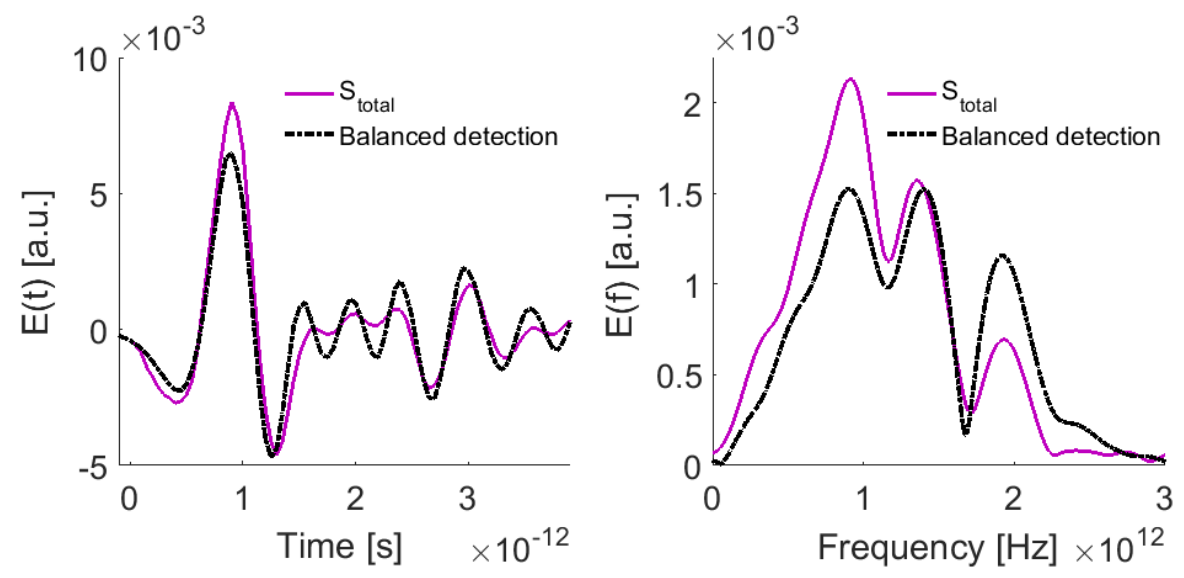

Figure 3.10: A comparison of the ambient air (45\% rel. hum.) THz pulse calculated by a linear combination of the two polarization components measured by single shot detection $\left(S_{\text {combo }}\right)$ and the full waveform measured by balanced detection, in the time domain (a) and in the frequency domain (b). We see significant differences suggesting there are are other issues in the single shot method.

spectrum suffers at frequencies above $\sim 1.5 \mathrm{THz}$

$$
S_{\text {total }}=c_{1} \times P_{s}+c_{2} \times P_{p}
$$

As the lack of simultaneous measurement of both polarizations seems to, at least, not be the only issue leading to differences in single and multi shot detection, I here discuss other possible reasons. The predicted pulse duration of the wavefront-tilted probe at the Fourier plane is, given the used experimental parameters, $\sim 190$ fs. This gives us a maximum resolved frequency of $\sim 2.6 \mathrm{THz}$ which satisfies the Nyquist-Shannon sampling theorem. The pulse duration at the Fourier plane is likely longer than predicted however, due to the various aberrations, such as from the off-axis curved focussing mirror, wavefront distortion and dispersion from the transmissive optics and ZnTe crystal the pulse experiences along the way. Moreover, the lack of signal above $2 \mathrm{THz}$ can be explained from the assumptions made when building the $\mathrm{THz}$ setup. As the $\mathrm{THz}$ beam is not collimated 


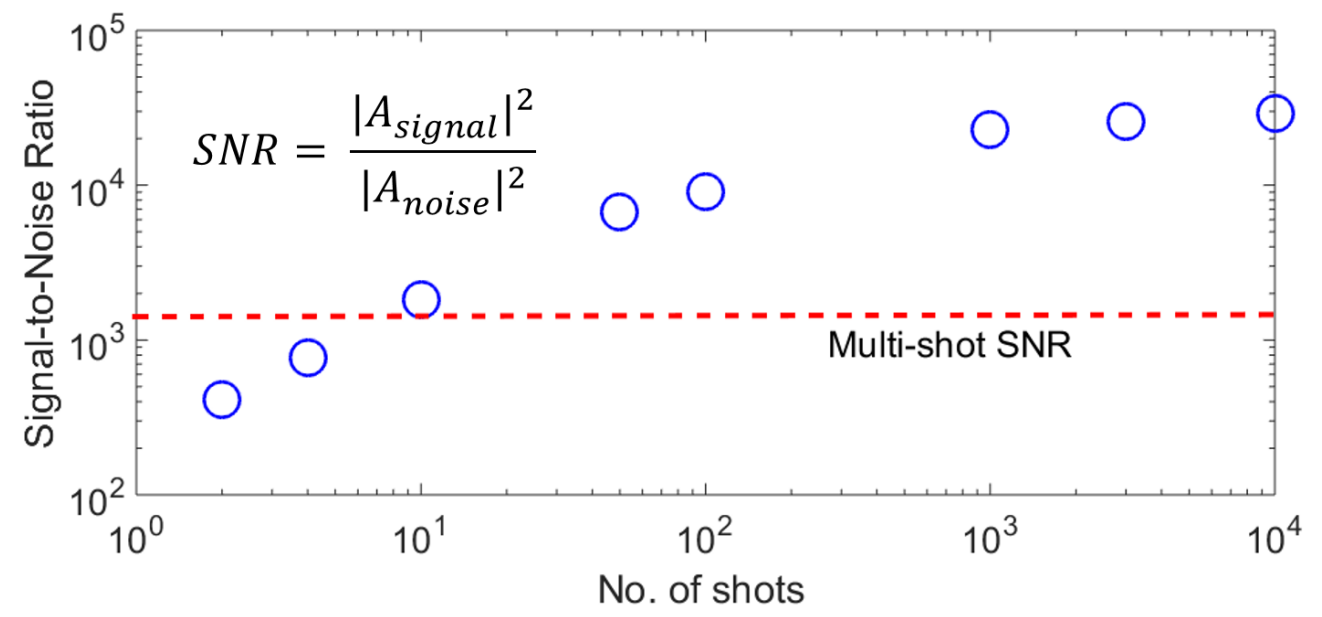

Figure 3.11: The SNR is given as a function of total number of shots. We see that 10 total shots, i.e. 5 averaged waveform spectra, have comparable sensitivity (SNR) to the multishot lock-in detection. However, over 300 shots (150 spectra) can be collected in the equivalent LIA measurement time, thus increasing sensitivity by an order of magnitude.

when incident on the first focussing off-axis parabolic (OAP) mirror. The mirror is set up in such a way as to focus a collimated beam, such as the $800 \mathrm{~nm}$ used to trace the beam path. Focussing a divergent beam will lead to highly aberrated, loosely focussed, and not collimated $\mathrm{THz}$ radiation. As we are mapping angle to time in the single shot detection method, and each $\mathrm{THz}$ frequency is emitted at a different angle [39], a non-focussed $\mathrm{THz}$ beam at the $\mathrm{ZnTe}$ detection signal can explain the lack of higher $\mathrm{THz}$ frequencies in the single shot detection.

In addition to the qualitative comparison of the spectra, we can also compare the sensitivity of the two techniques. As already noted in chapter 2, the sensitivity of the balanced detection technique was determined by a $\mathrm{SNR}=1.6 \times 10^{3}$, which was achieved with a $300 \mathrm{~ms}$ time constant on the LIA. The single shot waveform was collected at a different number of av- 
eraged laser shots, and the SNR was calculated for each. As the signal is calculated as a differential transmission, $\Delta \mathrm{T} / \mathrm{T}, 2$ sequential shots lead to one spectrum, i.e. 100 averaged shots corresponds to 50 averaged $\Delta \mathrm{T} / \mathrm{T}$ spectra. Averaged spectra of 2, 4, 10, 50, 100, 1000, 3000 and 10000 total shots were taken, and their SNR was plotted as a function of total no. of shots in Figure 3.11. We see that even using only 1 spectrum, i.e. a single pump on and a single pump off shot, we obtain a SNR of $>400$. The SNR of the multi-shot detection is given by the red dashed line for comparison. We see that already at 10 total shots, single shot detection has superior sensitivity to lock-in detection. To compare the measurement times, a 300 shot measurement can be achieved in the LIA time constant measurement time of $300 \mathrm{~ms}$, with an order of magnitude larger sensitivity. All of the arguments given above point to single shot measurement having a superior signal sensitivity to traditional lock-in multishot detection. This plays a crucial part in application to TRTS, where the excited state transient differential signal is measured, which commonly peaks on the order of $1-2 \%$ of the THz-TDS signal.

\subsection{Summary}

The entire $\mathrm{THz}$ waveform was collected in a single laser shot using a novel single-shot detection scheme based on a rotating probe and angle-to-time mapping. The probe signal was detected on a CMOS line camera. Singleshot detection of a single polarization component was achieved over a single shot time window of $5.7 \mathrm{ps}$. Comparisons of the single shot waveforms and spectra and those obtained by balanced detection show singleshot detection achieves much higher sensitivity, with the same SNR ratio as balanced detection achieved by only taking 10 laser shots, and measurements on the same time scale produce an order of magnitude better SNR. This method leads to remarkable reduction of measurement time, and directly leads to the viability of THz-TDS of irreversible systems and 
frequency-resolved TRTS. As such, it is a critical tool in allowing new experimental techniques and insights into transient carrier properties in optoelectronic materials.

However, data comparisons also show discrepancies between the two techniques, with an under-sampling of frequencies over $1 \mathrm{THz}$ in the single shot detection. These are strongly believed to come from aberrations and poor focussing of the $\mathrm{THz}$ pulse at the ZnTe detection crystal. The method can be vastly improved with better alignment and devising a method to capture both $s$ and $p$ polarization components. 



\section{Chapter 4}

\section{Single-shot Time Resolved Terahertz Spectroscopy}

\subsection{Introduction}

Charge carrier photogeneration, migration, regeneration as well as charge transfer and exciton formation all occur on ultrafast timescales. As a result, a non-contact probe of the transient photoconductivity with sub-picosecond time resolution is desirable [4]. Time-resolved terahertz spectroscopy (TRTS) is such a technique. An ultrafast pump-probe technique which utilizes terahertz pulses to directly probe the complex conductivity induced by optical excitations in a material from an optical pump pulse. TRTS is an increasingly popular tool for understanding charge carrier dynamics. TRTS has been used to probe the transient optical properties of various materials, ranging from traditional inorganic semiconductors [3, 4, 6, 19], 2D materials [50], organic semiconductors [4, 51, 52], polymers[53], and organic photovoltaic materials $[27,28,29]$ to metal halide perovskites $[54,55$, 56]. While already widely applicable, TRTS can be significantly improved upon, considering it is based on monitoring the time evolution of only a single peak. 
As previously discussed in chapter 3, performing TRTS across the entire time-domain $\mathrm{THz}$ field would allow time resolved measurements of the frequency domain electric field spectra. This would lead to direct measurement of the entire complex-valued optical properties of the sample at each pump-probe delay time. As a result, one could extract the time-resolved mobilities of free charge carriers and excitons in optoelectronic devices. There are instances in literature where this is achieved by taking the THzTDS spectrum at several optical pump - THz probe time delays [28, 29]. However, due to the time constraints of collecting a THz-TDS spectrum, only several time points can be collected. While significantly more informative than just a single peak TRTS scan, several hours are needed for a single measurement, and even then, only several conductivity time points can be measured giving discontinuous time resolved data. For this reason, TRTS is a perfect candidate for using single shot $\mathrm{THz}$ detection.

A TRTS setup requires only minor changes to an existing THz-TDS setup, and the setup of a time-resolved terahertz spectrometer is discussed. In this chapter we use the requisite changes and the single shot detection developed in chapter 3 to perform single shot THz-TDS and single shot full waveform TRTS on a n-silicon wafer as a sample in a proof-of-concept study.

\subsection{Setup parameters and practical considerations}

Modifying the THz-TDS setup developed in chapters 2 and 3 to allow for TRTS measurements is conceptually straightforward. The sample, placed at the back focal plane of the first OAP (see Figure 3.4), needs to be optically excited by visible light, and the optical chopper used to lock into the THz-TDS signal is now moved to chop the routed optical pump. A second mechanical delay stage, which in the current geometry offered a time delay range of $110 \mathrm{ps}$, was used to control the time delay between 
the optical pump and the $\mathrm{THz}$ pulse. The optical excitation light was generated from the $800 \mathrm{~nm}$ fundamental by an optical parametric amplifier. The OPA used in the TRTS setup is a commercial system, a TOPAS-C from Light-conversion. The TOPAS containes two $\beta$-Barium borate (BBO) crystals from two-stage amplification. The two crystals have tunable angles to achieve the phase matching condition for the desired wavelength. UV-vis region femtosecond pusles for sample excitation are generated by second harmonic generation or sum frequency generation[57]. $400 \mathrm{~nm}$ excitation light was exclusively used in the proof-of-concept TRTS measurements in this thesis, however the OPA setup allows broad tunability of the optical pump and thus, selective excitation of specific materials (for example, in blends) and optical modes. Due to the physical constraints of working in the THz purge box, routing the optical pump required significant consideration. As was shown previously in Figure 3.4, the optical pump was routed through the first OAP, and thus allowed for collinear excitation. An OAP with a $3 \mathrm{~mm}$ diameter hole parallel to the focussed beam (commercially available from Thorlabs; part no. MPD269-M01) was used.

However, this solution, while seemingly elegant, restricts the optical beam size. The OAP has a back side hole of $8 \mathrm{~mm}$ diameter, which tapers to 3 $\mathrm{mm}$ on the reflective surface, and as a result apertures the optical beam. The optical beam needs to be significantly bigger than the THz spot size at the sample, so all of the probed area is optically excited. This is an issue, considering the previously estimated $\mathrm{THz}$ spot size at the focal point (i.e. the sample) was $\sim 3 \mathrm{~mm}$. To increase the optical pump spot size at the sample, a short focal length $(f=30 \mathrm{~mm})$ lens was placed just outside the $\mathrm{THz}$ purge box at the back end of the OAP in the optical beam path. The lens focusses the beam at the back hole of the OAP and then diverges. While the $3 \mathrm{~mm}$ front face hole still apertures the beam to $3 \mathrm{~mm}$ diameter at the OAP surface, the beam is still diverging and expands to $\sim 8 \mathrm{~mm}$ diameter beam at the sample. This is depicted in Figure 4.1. 


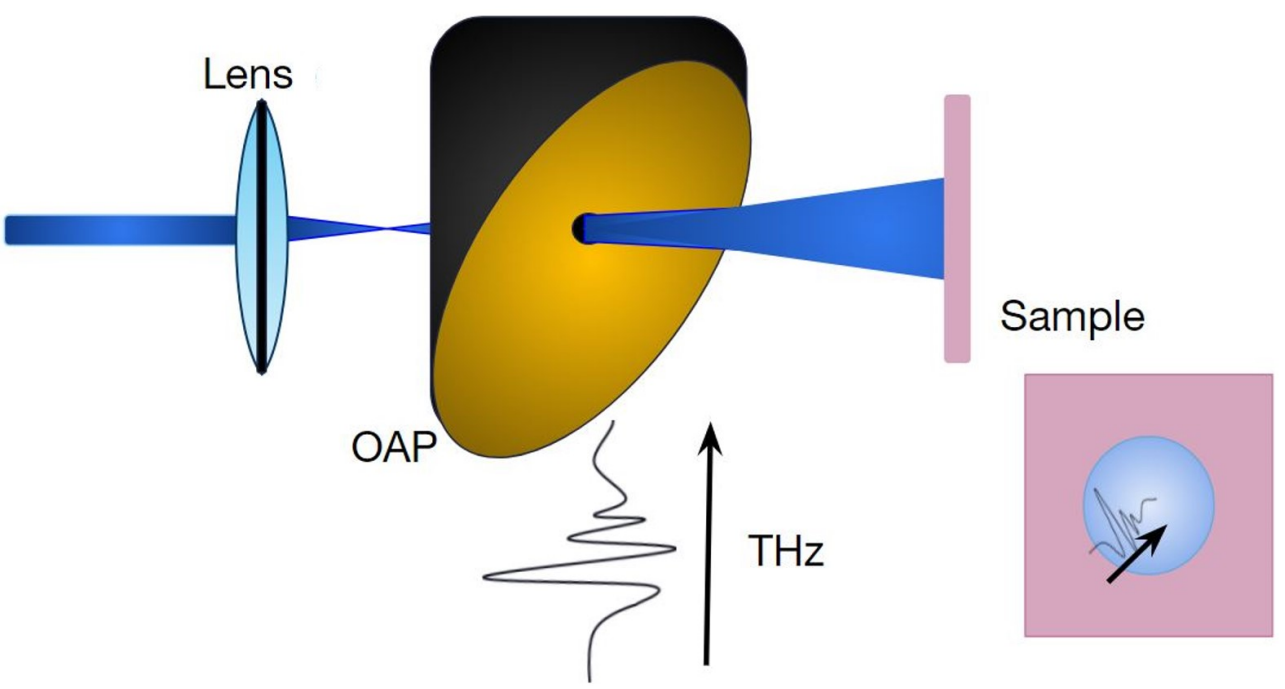

Figure 4.1: A schematic showing the implementation of the optical pump into the THz-TDS setup. A drilled hole ( $3 \mathrm{~mm}$ ) OAP is used allowing the optical pump to travel collinearly to the THz pulse. The optical pump beam spot at the sample is required to be larger than the $\mathrm{THz}$ spot at the focal point. To achieve this, a short focal length focussing lens is placed so that the beam focal point is just before entering the OAP. The beam then diverges and, while still apertured by the exit hole to $3 \mathrm{~mm}$, continues to diverge to $\sim 8 \mathrm{~mm}$ at the sample. The inset on the left shows a front face of the sample, with the $\mathrm{THz}$ incident on the excited area of the sample.

While the optical pump beam spot size at the sample is now significantly larger than the estimated $\mathrm{THz}$ spot size, this solution is inelegant and provides many possible issues. Firstly, the diverging beam makes it difficult to accurately measure the fluence and the beam spot size. This restricts making any quantitative conclusions from measurements. In addition, as the light is apertured by the $\mathrm{OAP}$, the optical pump intensity profile at the sample is inhomogeneous due to diffraction effects. As a result, it is difficult to convincingly claim that the entire $\mathrm{THz}$ spot size probes an equally excited part of the sample. While the implementation of the op- 
tical pump can clearly be a source of many uncertainty and issues, spatial and time constraints did not allow an alternative in the current iteration of the setup. A potential solution could be to have a non-linear crossing angle between the optical pump and THz, i.e. to come from the side of the OAP, not through it. The collinear arrangement can be sustained if the $\mathrm{THz}$ beam is focussed tighter, as discussed in Chapter 5 .

\subsection{Single-shot THz-TDS of Silicon}

Before running a TRTS experiment, a THz-TDS measurement of the sample is always performed first. THz-TDS allows us to first find the $\mathrm{THz}$ pump - $800 \mathrm{~nm}$ probe time delay at which we want to investigate the time-resolved behaviour, as well as giving the steady state, here meaning not optically excited, THz signal of the sample. As we want to perform a single-shot TRTS measurement, a single-shot THz-TDS of the sample is done first. Here we perform single-shot THz spectroscopy on a 500 $\mu \mathrm{m}$ thick sample of undoped [100] n-Silicon $(\mathrm{R}>1000 \Omega . \mathrm{cm})$ in a proof-ofconcept study.

The THz-TDS was first performed in multishot mode, in ambient conditions, to easily find the time delay associated with the $\mathrm{THz}$ pulse travelling through the $500 \mu \mathrm{m}$ thick sample. Using the multishot method, a long range scan can be used, and a scan over $>20$ ps around the reference air peak was performed. The $\mathrm{THz}$ time domain spectrum obtained with the silicon sample in place, as well as the associated reference waveform, is given in Figure 4.2. We see a time shift of $\Delta t=4.05$ ps between the reference and silicon spectra, as well as attenuation of the intensity due to mainly reflective losses. Float zone silicon has very low absorption across the entire $\mathrm{THz}$ range and is known to have been used a beam splitter for $\mathrm{THz}$ radiation [3, 6], so significant reflection losses are anticipated. The intensity attenuation is more obvious in the frequency spectra of the two 


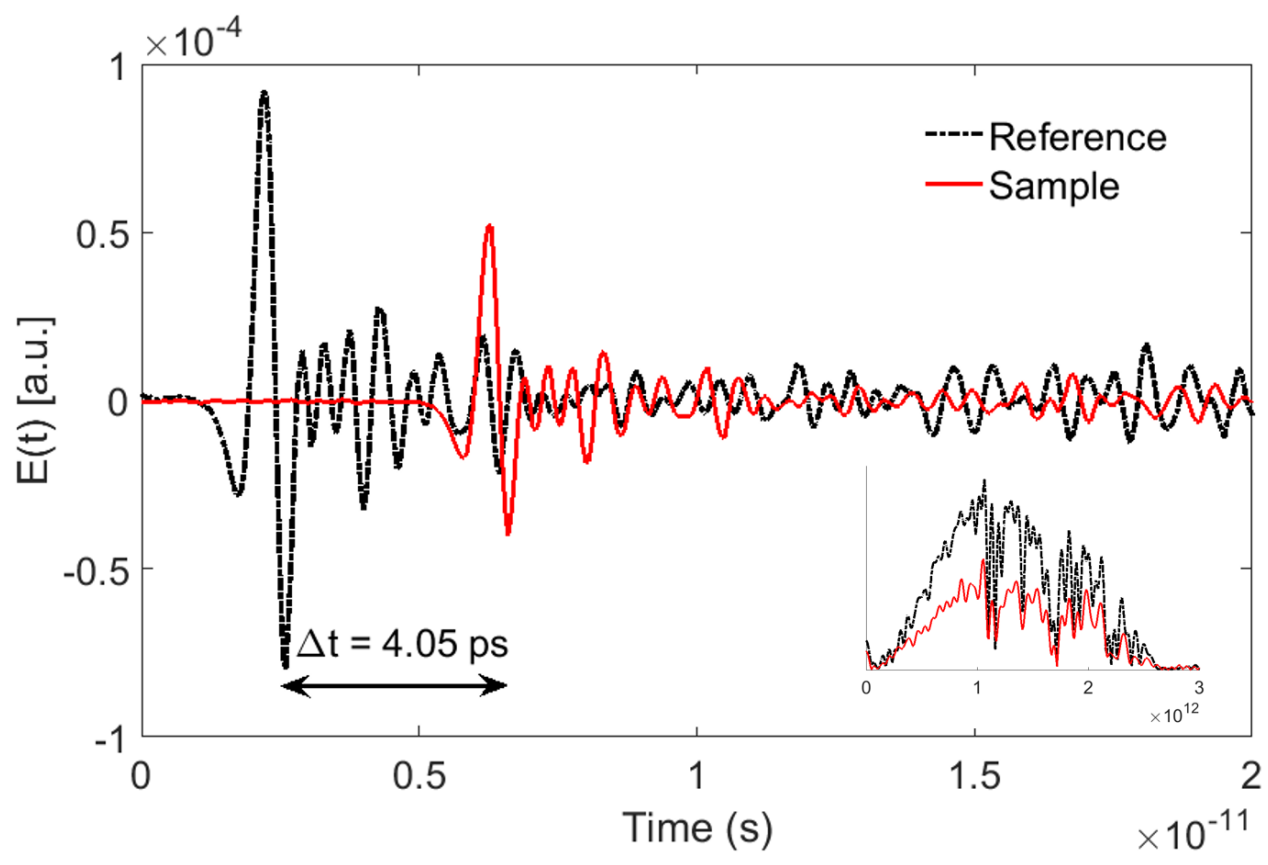

Figure 4.2: The THz-TDS time domain waveforms of silicon (red) and an air reference (black, dashed) collected in ambient atmosphere with balanced detection. Shows a relative time delay of 4.05 ps between the two signals. Inset: The respective frequency spectra.

waveforms, given in the inset of Figure 4.2.

From the time delay information obtained from Figure 4.2, we can now accurately move the $\mathrm{THz}$ probe delay stage between single shot measurements for accurate time window comparisons. In addition, we know that we require a larger time window, as the 4.05 ps need to be added. As a result, the single-shot waveform of the reference, this time in a low humidity nitrogen atmosphere $(4 \% \mathrm{RH})$, was collected first. The silicon wafer was then placed in the sample position and a single-shot THz-TDS measurement was taken without changing the probe time delay. This gives accurate pre-signal data for Silicon. The delay stage was then moved by 4.05 ps, and a second single-shot THz-TDS measurement for silicon was 
(a)

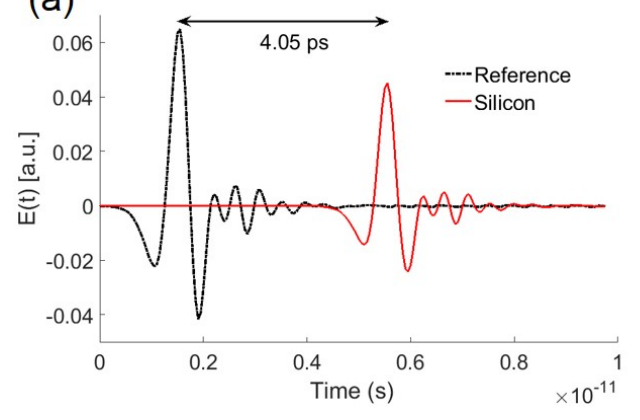

(b)

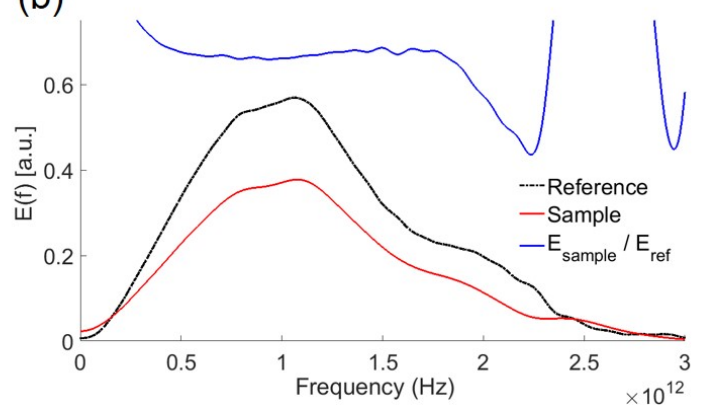

Figure 4.3: (a): The THz-TDS time domain waveforms of silicon (red) and an air reference (black, dashed) collected in a $\mathrm{N}_{2}$ atmosphere with single-shot detection. (b): The respective frequency spectra of the two waveforms given in (a). The ratio between the two spectra at each frequency is also given to show any spectral shifts or absorptions.

recorded. The data in the overlapping region (between 4.05 and $5.725 \mathrm{ps)}$ was taken from the second measurement to guarantee that the same parts of the probe beam profile were used to measure equivalent points in the silicon and reference waveforms. This assures that any spectral differences between the two waveforms do not occur due to small differences in the probe intensity profile across the pixel range. The resulting single-shot waveforms are given in Figure 4.3(a). The corresponding frequency spectra are given in Figure 4.3(b). In addition to the two spectra, the ratio of $E_{\text {sample }}(\mathrm{f})$ and $E_{\text {ref }}(\mathrm{f})$ at each frequency is also given. This can give a more obvious indication of any spectral shifts and absorptions. For Silicon, we see a reasonably flat attenuation ratio over the $0.5-1.8 \mathrm{THz}$ region. The features at $<0.5 \mathrm{THz}$ and $>1.8 \mathrm{THz}$ are exaggerated by the low electric field at these frequencies.

THZ-TDS is used to obtain the optical properties of a sample material. Following the method described in reference [13], the time domain and frequency spectra in Figure 4.3 were used to extract the complex-valued refractive index, $\hat{n}=n+i k$, of the n-silicon sample. The refractive index, 

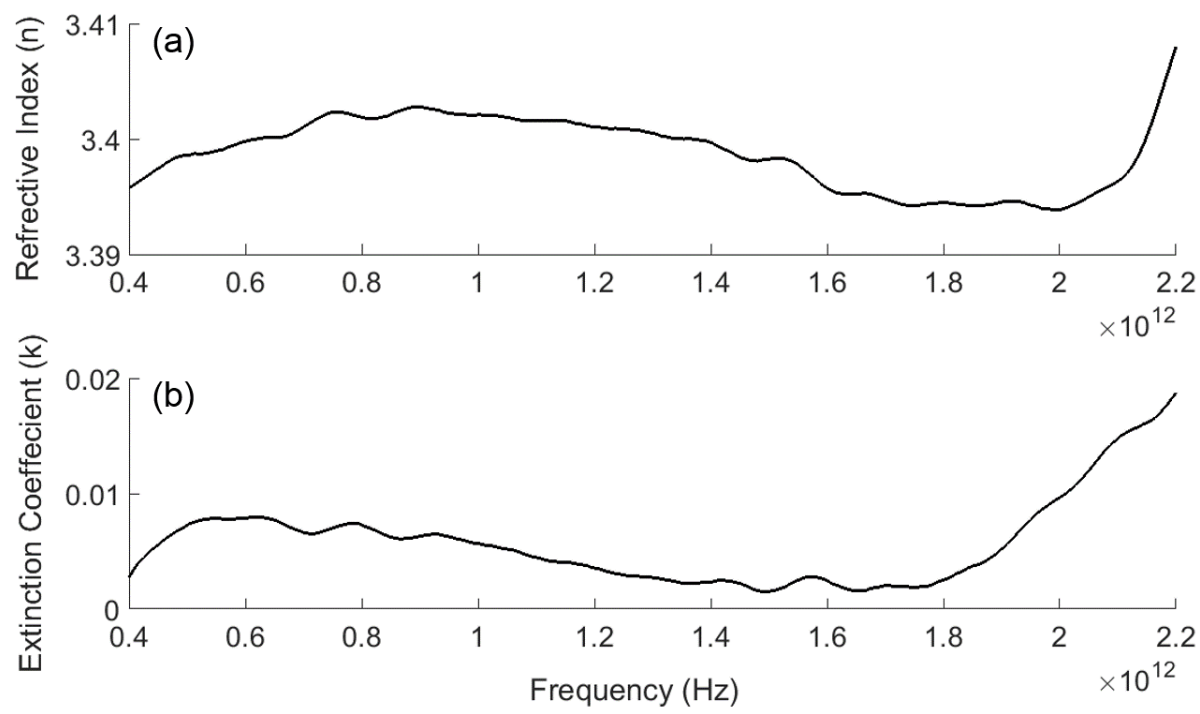

Figure 4.4: The optical properties of the n-silicon wafer extracted from the THTDS waveforms, here we give the frequency-dependant (a) refractive index and (b) The extinction coefficient, over the 0.4-2.2 THz region.

$n$, and extinction coefficient, $k$, are given in Figures 4.4 (a) and (b) respectively. The reasonably flat refractive index of $n \approx 3.395$ across the 0.4-2 $\mathrm{THz}$ region agrees with literature values[58, 59, 60, 61], as does the measured value of the extinction coefficient $k[59,60,61]$. If both $n$ and $k$, i.e. the entire complex valued refractive index, are known for the sample, then the optical properties of the material can alternatively be expressed as the complex dielectric constant $\hat{\varepsilon}(\omega)$ or complex conductivity $\hat{\sigma}(\omega)$ using equations 4.1-4.6:

$$
\begin{gathered}
\hat{\varepsilon}=\varepsilon_{1}+i \varepsilon_{2}=\hat{n}^{2} \\
\varepsilon_{1}=n^{2}-k^{2} \\
\varepsilon_{2}=2 n k \\
\hat{\sigma}=\sigma_{1}+i \sigma_{2} \\
\sigma_{1}=\varepsilon_{2} \varepsilon_{0} \omega
\end{gathered}
$$




$$
\sigma_{2}=-\left(\varepsilon_{1}-1\right) \varepsilon_{0} \omega
$$

where $\varepsilon_{0}$ is the permittivity of free space and $\omega=2 \pi \mathrm{f}$. These however, are all equivalent, and the fact that THz-TDS allows us to extract all of these optical properties directly highlights its effectiveness as a spectroscopic technique. As discussed in chapter 3, there are several issues with the developed single shot setup, which constrain its use for convincing quantitative and qualitative findings. While solving these issues is a priority for future work and is discussed in chapter 5, we see that even this iteration of the single shot spectrometer can give results for optical properties which are in agreement with those presented in literature. As the THz-TDS spectrum has now been collected, TRTS can further be performed to probe the excited state properties of the material.

\subsection{Single-shot TRTS of Silicon}

Upon performing THz-TDS on the silicon wafer, the optical pump was made incident via the method described in section 4.2. The optical chopper was moved from the THz pump beam path to the optical pump beam path. A variable ND filter allowed easy adjustment of the optical pump power. Spatial overlap of the optical pump and $\mathrm{THz}$ pulse was estimated using the $800 \mathrm{~nm} \mathrm{THz}$ pump as a THz proxy. The silicon sample allowed for an easy choice for finding an initial signal. As silicon has a long-lived carrier lifetime (on the order of $\sim 100 \mu \mathrm{s}$ [62]), the delay stage was set to the furthest forward position which corresponds to the latest possible time, i.e. the optical pump arriving as early as possible. Due to this long lifetime, the signal search was performed at late time rather than the more standard method of searching around the calculated nominal $t_{0}$, when the two beams reach the sample simultaneously. Due to the implementation of the optical pump beam, where a diverging beam is used, the pump intensity is significantly lowered. As a result, the pump intensity was maximised in an attempt to create enough charge carriers to observe a pump- 
induced change. Once the signal was found, the spatial overlap was adjusted to maximise signal strength, and the fluence was adjusted to $\sim 40$ $\mathrm{nJ} / \mathrm{cm}^{2} /$ pulse at which the signal strength was maximal. Due to the inhomogeneity of the optical pump beam at the sample (as discussed in section $4.2)$, this is likely only an average fluence, and could vary significantly. The signal onset time, $t_{0}$, was then found by moving the stage back and monitoring the signal strength. For the measurement itself, each pump-probe delay time single shot spectrum was averaged 10000 times, and the measurement was taken over a $>100 \mathrm{ps}$ range. Delay time points were chosen to be unevenly spread over the experimental time range. Increments on the order of $10 \mathrm{fs}$ where used around $t_{0}$, where initial fast dynamics are expected, whereas the time between points increased (incrementally, up to several ps) at late times where fast kinetic changes are unlikely. As a result, a total of 201 time-points were collected in a measurement that took just over 40 minutes, a remarkable reduction in time compared to any dual delay setup.

The obtained data was plotted as a surface in Figure 4.5(a), with the measured electric field signal (measured as $\Delta \mathrm{T} / \mathrm{T}$ ) given as a function of both camera pixel and optical pump - THz probe delay time. From the falsecolour map we see that the signal is long lived and, as expected, is present over the entire time delay range measured. The representation of data in this format is convenient as now a slice along the vertical axes will give a single shot TDS waveform at the designated time delay and a slice along the horizontal axis gives a kinetic trace of the time evolution of that part of the waveform. An optical pump induced TDS waveform averaged from those obtained between 20 and 25 ps of time delay is given in Figure 4.5(b). By averaging over multiple time points, the sensitivity is further increased. One must be careful that only regions where the signal strength is similar are averaged, otherwise erroneous results are possible. To avoid this, the surface can be consulted for regions used to average. We see that we fully 

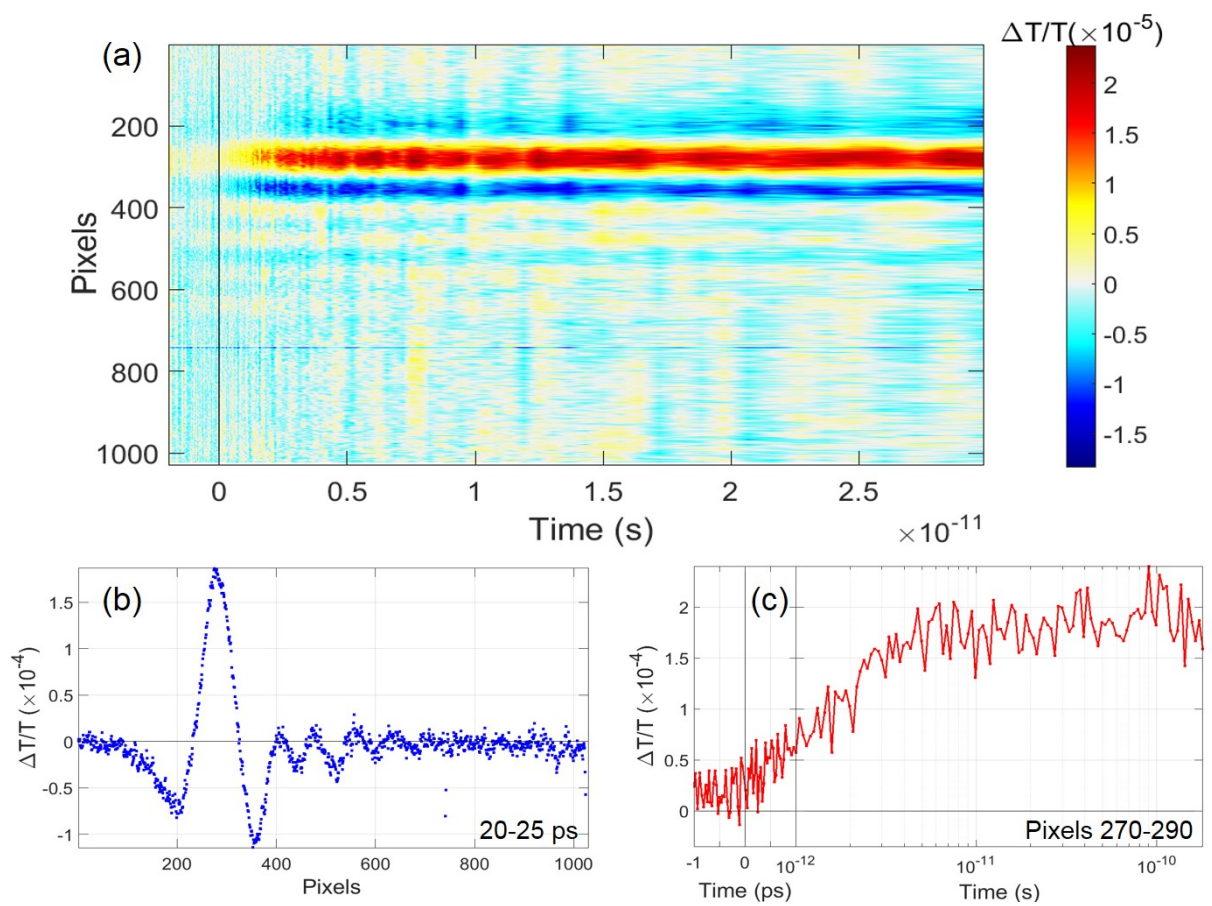

Figure 4.5: (a) The time-resolved single shot time-domain $\mathrm{THz}$ surface from $\mathrm{n}$ silicon excited at $400 \mathrm{~nm}$ at $\sim 40 \mathrm{~nJ} / \mathrm{cm}^{2} /$ pulse. (b) The optical-pump induced $\Delta \mathrm{E}(\mathrm{t})$ single shot waveform averaged over the 20-25 ps time points. (c) The kinetics of the optical-pump induced $\Delta \mathrm{E}(\mathrm{t})$ of the peak signal averaged over pixels 270-290.

resolve not only the waveform peak, but multiple peaks after the signal maximum. This gives encouragement to the notion that this technique can monitor the time evolution of any part of the waveform, and thus the full frequency spectrum. A kinetic trace averaged from 10 pixels either side of the peak pixel is given in Figure 4.5(c). We see a signal rise on the order of $\sim 5$ ps followed by a long lived flat signal. Considering the experimental time window used ( $110 \mathrm{ps}$ ) and the long lifetime associated with silicon, it was not possible to extract a carrier lifetime from the silicon time dependence measurement. The kinetic trace taken at the peak amplitude is the information one would obtain from traditional TRTS. Here we have the ability to look at the time evolution of any part of the waveform in the 

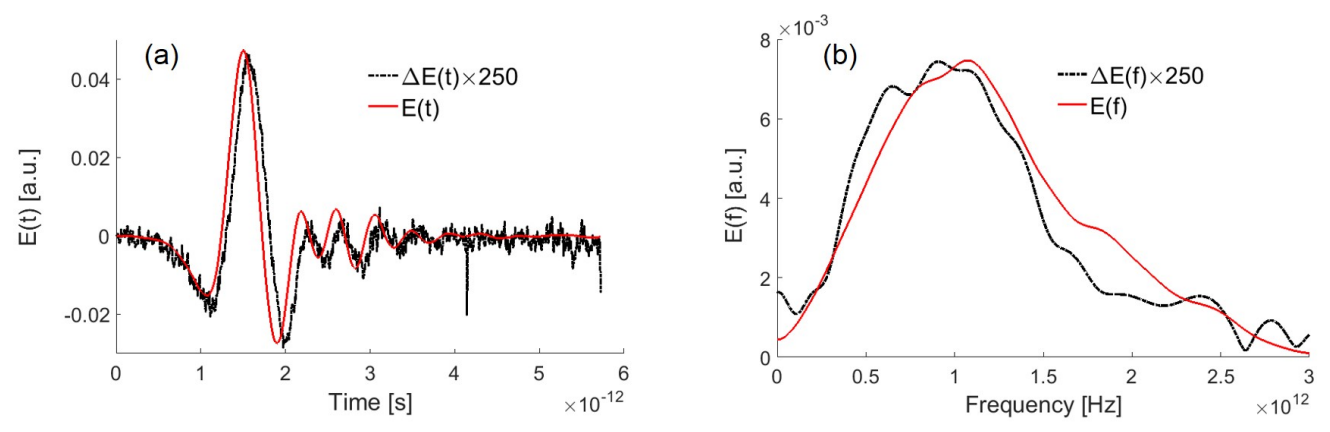

Figure 4.6: A comparison of the pump induced signal change and the steady state THz-TDS of the silicon sample. In the time domain (a), a small phase shift of $\Delta \mathrm{E}(\mathrm{t})$ to later time of $\sim 100 \mathrm{fs}$ is observed, while in the frequency domain (b) a sharper cut-off at $>1.5 \mathrm{THz}$ is observed for $\Delta \mathrm{E}(\mathrm{f})$.

single shot time window. Silicon only shows an increase in photocarriers, and thus a higher reflection of the THz occurs. This leads to a bleaching of the THz signal compared to the ground state, which is observed here. Due to the long-lived silicon photocarriers, taking spectral slices at different times is not informative in this case, as unfortunately no spectral shifts are observed in the TRTS surface. As a result, silicon is not an ideal sample to demonstrate the full potential of single shot TRTS, but is still an adequate proof-of-concept study.

We can now take the optically induced data and compare it to the steady state TDS obtained in section 4.3. First the pixel axis needs to be changed to the time domain via the pixel-to-time calibration outlined in chapter 3. The $\Delta \mathrm{E}(\mathrm{t})$ signal was then scaled to be comparable to the steady state TDS signal. The two waveforms are plotted in Figure 4.3(a). We see a small phase shift to later time of $\sim 100 \mathrm{fs}$, representing the change in refractive index of the optically excited silicon. Otherwise we see that we are able to resolve all of the major peaks in the time domain. In addition, we see that the differential spectrum is $\sim 250$ times smaller than the pump off signal. Standard TRTS signals are on the order of $1-2 \%$, and the fact that the ob- 

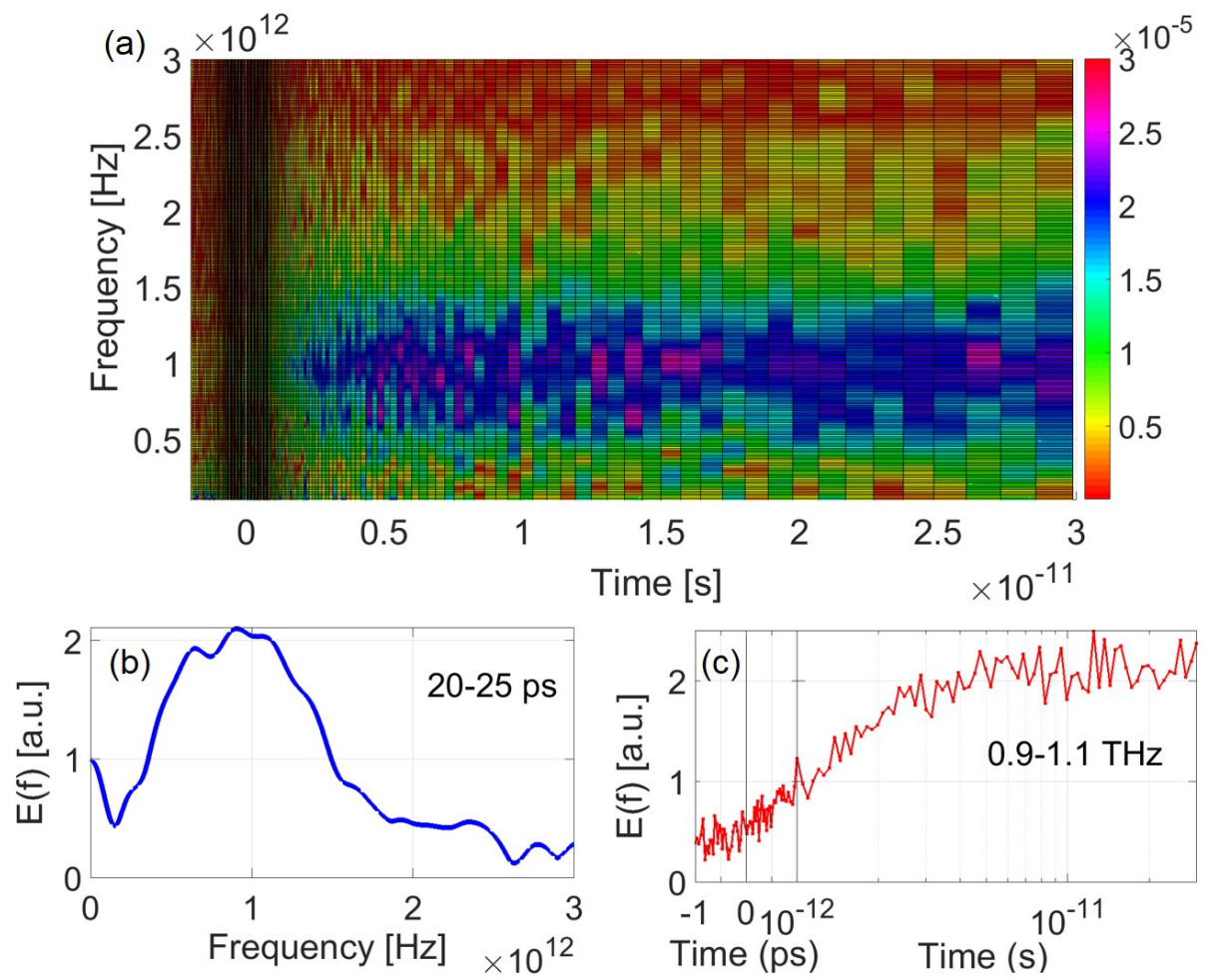

Figure 4.7: (a) The time-resolved single shot frequency-domain THz surface from $\mathrm{n}$-silicon excited at $400 \mathrm{~nm}$ at $\sim 40 \mathrm{~nJ} / \mathrm{cm}^{2} /$ pulse. (b) The optical-pump induced $\Delta \mathrm{E}$ (f) spectrum averaged over the $20-25$ ps time points. (c) The kinetics of the optical-pump induced $\Delta \mathrm{E}(\mathrm{f})$ of the spectrum averaged over 0.9-1.1 THz.

served signal is significantly smaller could point to poor optical pump $\mathrm{THz}$ overlap, or that due to the diverging beam, the pump intensity is not high enough for a maximum $\Delta \mathrm{E}(\mathrm{t})$ signal. The respective frequency spectra, obtained by Fourier transform, are given in Figure 4.3(b). The $\Delta \mathrm{E}(\mathrm{f})$ signal seems to have a sharper intensity drop at $\sim 1.4$, but again the single shot detection needs to be optimized to make any conclusive quantitative arguments.

Obtaining the optical induced frequency-domain electric field is one of the main advantages of single shot full waveform TRTS. Here we can mon- 
itor the evolution of spectral features in the frequency domain. This is not possible in traditional TRTS, as the entire waveform is required to obtain the Fourier spectra. As a result, it might be of interest to present the data as a surface of time-resolved frequency-domain spectra. The time domain waveform at each pump probe time delay was transformed to the respective frequency spectrum. The time-dependant frequency domain spectral surface is given in Figure 4.7(a). Again horizontal and vertical slices can be taken, except now the frequency-domain electric field is monitored. The same 20-25 ps spectral average as previously done, is taken for completion (Figure 4.7(b)), giving the same spectrum as in 4.3(b). A kinetic trace of the time evolution of the $1 \mathrm{THz}$ peak, averaged over the 0.9-1.1 THz spectral region is given in Figure 4.7(c), showing the same rise time.

While the silicon sample presented here has very simple excited state dynamics, the proof-of-concept study serves to present the application of single shot TRTS. This technique can easily be improved upon to the point where quantitative single shot TRTS would be able to accurately measure the pump induced complex conductivity for each frequency spectrum, and hence extract the mobility at each pump-probe delay point. Thus, the results shown in this chapter indicate a direct measurement of timeresolved mobility, with $\mathrm{THz}$ and its ability to distinguish between excitons and free charges, is achievable by utilising the proposed single-shot $\mathrm{THz}$ detection scheme. 


\section{Chapter 5}

\section{Future Work and Proposed Improvements}

While the developed THz-TDS and TRTS setup shows great promise, it requires several improvements in order to perform quantitative, convincing single shot full waveform THz-TDS and TRTS. Several design changes are proposed here that I believe will to lead to single shot detection which will quantitatively match the results from traditional balanced detection. In addition, they are expected to lead to higher efficiency $\mathrm{THz}$ generation, better optical pump integration and increased $\mathrm{THz}$ bandwidth.

The most stark, and possibly most important, improvement can be made by changing the 3 OAP design of the THz propagation arm of the experiment. In the current setup, as detailed in chapter 2, a collimated $800 \mathrm{~nm}$ beam is used to generate $\mathrm{THz}$ radiation in a ZnTe crystal by optical rectification. The setup is based on the assumption that the $\mathrm{THz}$ generated travels as the $800 \mathrm{~nm}$ seed through the pump arm of the experiment, and thus, that the $\mathrm{THz}$ is collimated upon generation. In the current setup, the first OAP is used to focus the collimated beam into the sample. Off-axis parabolic mirrors only focus tightly when oriented correctly toward either a collimated beam or a beam focussed at the focal point (i.e. a quasi-point 
source)[63]. Upon generation, the THz beam diverges from the crystal[39]. Attempting to focus a diverging beam will lead to $\mathrm{THz}$ which is not collimated, highly abberated and not focussed at the current expected focal point. This is observed when measuring the $\mathrm{THz}$ beam size at the focal point. We measure the $\mathrm{THz}$ beam size to be $\sim 3 \mathrm{~mm}$ in diameter, which is an order of magnitude larger than the diffraction limited beam size [64]. As the $\mathrm{THz}$ pulse is broadband (spans a significant wavelength range), different frequency components are found in different spatial parts of the propagating beam, and thus in different parts of the "focussed" beam spot. In addition, each $\mathrm{THz}$ frequency is emitted at a different angle and any aberrations and lack of tight focussing either at the sample or at the ZnTe detection crystal could in fact be the sole reason for the inability of the single-shot detection to sample at $\mathrm{THz}$ frequencies above $2 \mathrm{THz}$, as even pessimistic predictions suggest sampling over $2 \mathrm{THz}$ is possible (Nyquist-Shannon sampling theorem). The proposed solution is reasonably straightforward, and would be to use a 4 OAP mirror setup, where the first OAP would be set in the orientation to collimate a point source diverging beam, the second mirror would then tightly focus the collimated $\mathrm{THz}$ radiation into the sample, which would be re-collimated with the third OAP, and the last OAP would again have the role of focussing the $\mathrm{THz}$ into the $\mathrm{ZnTe}$ detection crystal. The revised OAP setup is depicted schematically in Figure 5.1. To align the setup with the $800 \mathrm{~nm}$ beam, a focussing element should be placed to focus the 800 beam at the ZnTe generation crystal position to simulate a point source. Indeed, generating $\mathrm{THz}$ with a focussed beam would likely allow for better focussing with the OAPs and it has even been suggested that using an excitation spot comparable to the $\mathrm{THz}$ wavelengths being generated is further beneficial[39]. This is another change that should be made, as long as the generation fluence is managed so as to not lead to two-photon absorption.

The current poor focussing of the $\mathrm{THz}$ at the sample in turn creates the 


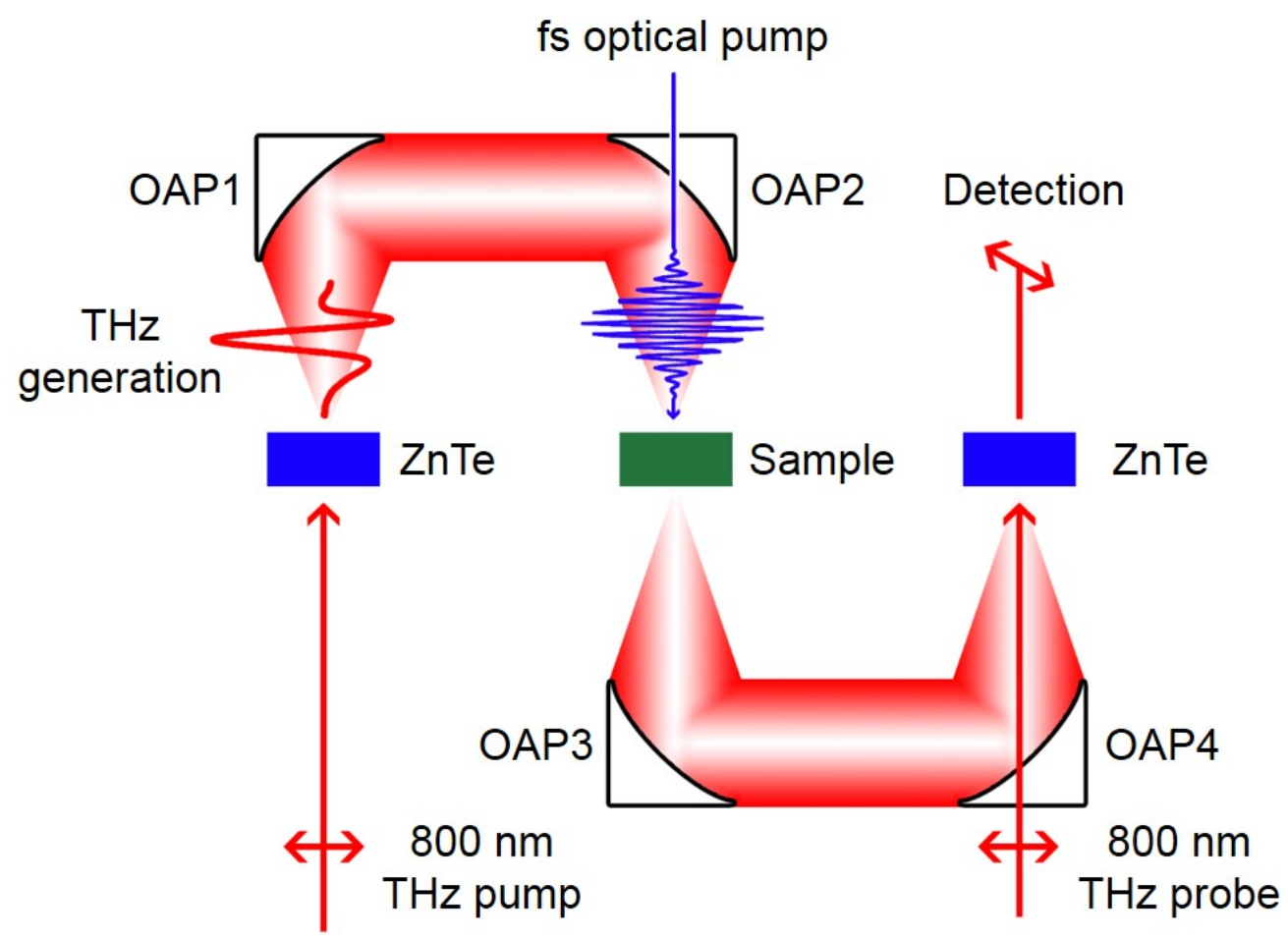

Figure 5.1: A schematic showing the proposed change to the OAP setup, moving from 3 to 4 OAPs. This new setup should allow tight focussing of the THz both at the sample and at the detection crystal.

issue with the optical pump excitation, which needs to be magnified from the starting $3 \mathrm{~mm}$ diameter in order to excite a large enough area of the sample. If a tightly focussed $\mathrm{THz}$ beam at the sample was achieved, no alteration of the optical pump would be needed, relinquishing the need for a diverging and inhomogeneous pump beam. An unaltered optical pump beam would allow for quantitative fluence-dependent measurements.

Another potential improvement would be to set up the spectrometer in reflection mode. Some samples, especially those with high carrier concentrations, reflect most of the $\mathrm{THz}$ signal, and would require a reflection mode measurement. An ideal design would allow switching between transmis- 
sion and reflection mode easily, without much adjustment to the beam path and optics, depending on the sample of interest. If the above changes to the OAPs is made, placing a pair of flat mirrors in the area where the beam is focussed into the sample (between the second and third OAP) would enable reflection measurements.

At the moment, we are creating a time window of $\sim 20$ ps with a large diameter probe, but are aperturing the beam to only measure a $\sim 5.7 \mathrm{ps}$ time window with single shot detection. The beam at the image plane can be optically relayed, and demagnified at the same point, in order to have a larger part of the beam on the camera sensor. As the pixel resolution can be ignored (A pixel corresponds to $5.6 \mathrm{fs}$ yet the pulse width is $\sim 200 \mathrm{fs}$ ), this would not reduce the time resolution at all while giving a better time window.

Another significant improvement would be to collect both the $s$ and $p$ polarizations in single shot mode. This can be done by either using two synchronised cameras, which requires customized equipment, or fitting both polarizations on the camera. As shown the pixel resolution is good enough for "compressing" the waveform in less pixels, this is achievable but is practically difficult. It can be done, however, by relaying the image plane, demagnifying the probe and using a polarizing beam displacer. Future work should investigate methods of measuring both polarization components as it would increase signal strength and accuracy.

Finally, the single optical chopper setup currently requires a separate THzTDS measurement before performing TRTS. The THz-TDS measures the signal due to the THz pump, while the TRTS measures only the optical pump induced signal. However, a 2 chopper system would allow the measurement of both at the same time, leading to a better comparison. For the $3000 \mathrm{~Hz}$ laser system, one chopper would chop the THz pump at 1500 
$\mathrm{Hz}$, and the second chopper would chop the optical pump at $750 \mathrm{~Hz}$. As a result, we would have 4 different types of shots: THz pump off/optical pump off shots $\left(T_{00}\right)$, THz on/optical pump off shots $\left(T_{10}\right)$, THz off/optical pump on shots $\left(T_{01}\right)$ and on/on shots $\left(T_{11}\right)$. With proper shot indexing, $\left(T_{10}-T_{00}\right) / T_{00}$ shots can be used to calculate the THz-TDS data. This additionally provides a THz-TDS spectrum for more accurate comparison than those obtained from a separate THz-TDS measurement, as $\mathrm{THz}$ can be sensitive to minor changes in humidity and temperature, as well as the beam pointing controlling signal overlap. $\left(T_{11}-T_{10}\right) / T_{10}$ would give the TRTS signal as measured in chapter 4 . The $T_{01}$ shots can be discarded as they provide no additional data.

Ultimately, the single-shot TRTS (SS-TRTS) setup, once optimized, should be used as a standard complement to other in-house spectroscopic methods, such as Transient Absorption (TA), Ultrafast Photoluminescence (PL) and Ultrafast Raman spectroscopy. Many novel organic materials have been proposed for OPV applications due to their high quantum yields and mobilities. SS-TRTS would allow for conclusive arguments regarding the sources of mobility, and the role of excitons and free charge carriers in new generation materials, and can be used to probe a wide range of materials, from organic semiconductors to perovskites and 2D materials. While certainly useful as a complement to other measurements, I believe that SS-TRTS, with its ability to directly probe the transient complex photoconductivity, can itself lead to completely new insights on the mobilities in these systems. 



\section{Chapter 6}

\section{Conclusions}

\subsection{THz-TDS spectroscopy}

In this thesis a time-domain terahertz spectrometer was built and characterized. A broadband 0 - 2.6 THz pulse was generated by optical rectification in a [110] ZnTe crystal and detected by EO free-space sampling with an identical ZnTe crystal, balanced photodiodes and a Lock-in Amplifier. The effect of ZnTe angle compared to the probe polarisation was investigated and optimized. The effect of atmospheric water was also investigated and showed that measurements need to be carried out in a low-humidity atmosphere as the water absorption is extremely intense and will dominate, and thus mask, most spectral shifts throughout the 1-2.5 THz region. A peak SNR of $\sim 1600$ was achieved with a reasonable LIA time constant of $300 \mathrm{~ms}$, which allows resolution of the entire $\mathrm{THz}$ waveform including multiple pulse reflections. The developed THz-TDS spectrometer can be used to obtain the steady-state optical properties of a sample, such as the complex refractive index, dielectric function and con-

"If all the economists in the world were laid end-to-end they wouldn't reach a conclusion, and neither shall I." - George Bernard Shaw 
ductivity, in the $\mathrm{THz}$ frequency region. While improvements suggested in chapter 5 could lead to a stronger $\mathrm{THz}$ signal and an extension of the bandwidth, the developped THz-TDS spectrometer already provides high quality SNR and reproducible data. However, once the single-shot is improved to qualitatively match the balanced detection measurement, traditional balanced detection THz-TDS will be obsolete and replaced by the single-shot THz-TDS setup developed further in the thesis.

\subsection{Single shot THz-TDS}

The main motivation of the thesis was to collect the entire $\mathrm{THz}$ waveform in a single laser shot to allow for practical time resolved measurements of the full $\mathrm{THz}$ waveform. To that end, a novel single-shot detection scheme was developed. A transmissive grating was used to impart pulse-front tilt to the $800 \mathrm{~nm}$ probe, which was rotated around the back focal point in the ZnTe detection crystal, encoding time-to-angle. The encoding was undone by spreading the rotated probe over a linear detector. The probe signal was detected on a CMOS 1024 pixel camera. Single-shot detection of a single polarization component was achieved over a single shot time window of $5.7 \mathrm{ps}$. Comparisons of the single shot waveforms and spectra and those obtained by balanced detection show single-shot detection achieves much higher sensitivity, with the same SNR ratio as balanced detection achieved by only taking 10 laser shots. At a $3 \mathrm{kHz}$ repetition rate, these are collected in less than a millisecond, whereas the balanced detection measurement requires a sampling stage and takes $\sim 10$ minutes over the same time window. And while measurement time for balanced detection scales with number of time points, i.e. with time resolution and time window, the single shot only depends on the number of shots required to achieve a sufficient SNR. However, data comparisons also show discrepancies between the two techniques, with an under-sampling of frequencies over 1 $\mathrm{THz}$ in the single shot detection. These are strongly believed to come from 
aberrations and poor focussing of the $\mathrm{THz}$ pulse at the ZnTe detection crystal. The improvements suggested in chapter 5 are, however, expected to lead to a quantitative match between the two techniques. This would allow widely applicable use of the single shot detection technique, as a vastly superior detection method to balanced detection.

The single shot THz-TDS method was used to measure the optical properties of a n-Silicon wafer. The complex refractive index $\hat{n}$, i.e. the refractive index $n$ and the extinction coefficient $k$, were obtained. The obtained values largely agree with literature values.

\subsection{Single shot TRTS}

The developed single shot detection was implemented to achieve a full waveform single shot time resolved terahertz (optical pump - terahertz probe) measurement. The single shot time resolved terahertz spectroscopy (SS-TRTS) setup was used to measure the time dependent THz-TDS spectra of n-Silicon, in a proof-of-concept study. It is demonstrated that the entire pump-induced electric field can be resolved in the time domain, and a full spectral TRTS can be collected with no loss of time compared to traditional TRTS. The sensitivity of the single-shot method allows high SNR data, even for $\Delta \mathrm{E}(\mathrm{t})$, when high shot averaging is used. This allows a previously impractical measurement, with a measurement time of over 16 hours, to be performed quickly (on the order of 30 minutes), thus allowing its use for the direct study of exciton and free charge carrier mobilities.

The results presented in this thesis show that single shot TRTS is achievable by using a pulse-front tilt method, and allows for time resolved measurements of the entire $\mathrm{THz}$ time domain waveform, and thus the frequency domain spectrum. However, the fact that the single shot detection is not fully developed and does not match quantitatively with balanced 
detection limits further analysis of the current iteration of the setup. In addition to the detection, the implementation of the optical pump can be improved upon significantly, to allow for quantitative fluence measurement and a homogeneous excitation spot. After these improvements are made, single shot TRTS can be used a standard complement to other in-house spectroscopic methods, such as Transient Absorption (TA), Ultrafast Photoluminescence (PL) and Ultrafast Raman spectroscopies.

Due to its unique ability to probe the transient complex conductivity directly, the modification to traditional TRTS should make it the leading tool in charge mobility studies, and should lead to increased and widespread use of TRTS. In this thesis, the results prove complete single shot frequencyresolved TRTS is viable, adding a spectroscopic tool which can lead to new insights into charge mobilities in optoelectronic materials. 


\section{Bibliography}

[1] V. Ghergia, "New materials for optoelectronic devices," Ceramics international, vol. 19, no. 3, pp. 181-190, 1993.

[2] O. Ostroverkhova, "Organic optoelectronic materials: mechanisms and applications," Chemical reviews, vol. 116, no. 22, pp. 13279-13412, 2016.

[3] R. Ulbricht, E. Hendry, J. Shan, T. F. Heinz, and M. Bonn, "Carrier dynamics in semiconductors studied with time-resolved terahertz spectroscopy," Reviews of Modern Physics, vol. 83, no. 2, p. 543, 2011.

[4] G. Lanzani, Photophysics of molecular materials: from single molecules to single crystals. Wiley, 2006.

[5] E. Hendry, J. M. Schins, L. Candeias, L. Siebbeles, and M. Bonn, “Efficiency of exciton and charge carrier photogeneration in a semiconducting polymer," Physical review letters, vol. 92, no. 19, p. 196601, 2004.

[6] J. B. Baxter and G. W. Guglietta, "Terahertz spectroscopy," Analytical chemistry, vol. 83, no. 12, pp. 4342-4368, 2011.

[7] M. C. Nuss and J. Orenstein, "Terahertz time-domain spectroscopy," in Millimeter and submillimeter wave spectroscopy of solids, pp. 7-50, Springer, 1998.

[8] H. A. Kramers, La diffusion de la lumiere par les atomes. 1927. 
[9] R. d. Kronig, "Algemeene theorie der diëlectrische en magnetische verliezen," Ned. T. Natuurk, vol. 9, p. 402, 1942.

[10] C. F. Bohren, "What did Kramers and Kronig do and how did they do it?," Eur. J. Phys., vol. 31, p. 573, 2010.

[11] A. Djorović, M. Meyer, B. L. Darby, and E. C. Le Ru, "Accurate modeling of the polarizability of dyes for electromagnetic calculations," ACS Omega, vol. 2, no. 5, pp. 1804-1811, 2017.

[12] R. N. Bracewell and R. N. Bracewell, The Fourier transform and its applications, vol. 31999. McGraw-Hill New York, 1986.

[13] L. Duvillaret, F. Garet, and J.-L. Coutaz, "Highly precise determination of optical constants and sample thickness in terahertz timedomain spectroscopy," Applied optics, vol. 38, no. 2, pp. 409-415, 1999.

[14] Z.-H. Zhai, S.-C. Zhong, J. Li, L.-G. Zhu, K. Meng, J. Li, Q. Liu, Q.-X. Peng, Z.-R. Li, and J.-H. Zhao, "Time-resolved single-shot terahertz time-domain spectroscopy for ultrafast irreversible processes," Review of Scientific Instruments, vol. 87, no. 9, p. 095101, 2016.

[15] P. Uhd Jepsen, W. Schairer, I. Libon, U. Lemmer, N. Hecker, M. Birkholz, K. Lips, and M. Schall, "Ultrafast carrier trapping in microcrystalline silicon observed in optical pump-terahertz probe measurements," Applied Physics Letters, vol. 79, no. 9, pp. 1291-1293, 2001.

[16] P. A. George, J. Strait, J. Dawlaty, S. Shivaraman, M. Chandrashekhar, F. Rana, and M. G. Spencer, "Ultrafast optical-pump terahertz-probe spectroscopy of the carrier relaxation and recombination dynamics in epitaxial graphene," Nano letters, vol. 8, no. 12, pp. 4248-4251, 2008.

[17] J. H. Strait, H. Wang, S. Shivaraman, V. Shields, M. Spencer, and F. Rana, "Very slow cooling dynamics of photoexcited carriers in 
graphene observed by optical-pump terahertz-probe spectroscopy," Nano letters, vol. 11, no. 11, pp. 4902-4906, 2011.

[18] M. C. Beard, G. M. Turner, and C. A. Schmuttenmaer, “Transient photoconductivity in gaas as measured by time-resolved terahertz spectroscopy," Physical Review B, vol. 62, no. 23, p. 15764, 2000.

[19] D. A. Yarotski, R. D. Averitt, N. Negre, S. A. Crooker, A. J. Taylor, G. P. Donati, A. Stintz, L. F. Lester, and K. J. Malloy, "Ultrafast carrier-relaxation dynamics in self-assembled inas/gaas quantum dots," JOSA B, vol. 19, no. 6, pp. 1480-1484, 2002.

[20] S. M. Teo, B. K. Ofori-Okai, C. A. Werley, and K. A. Nelson, “Invited article: Single-shot thz detection techniques optimized for multidimensional thz spectroscopy," Review of Scientific Instruments, vol. 86, no. 5, p. 051301, 2015.

[21] E. Knoesel, M. Bonn, J. Shan, and T. Heinz, "Charge transport and carrier dynamics in liquids probed by thz time-domain spectroscopy," Physical review letters, vol. 86, no. 2, p. 340, 2001.

[22] R. Groeneveld and D. Grischkowskyt, "Picosecond time-resolved farinfrared experiments on carriers and excitons in gaas-algaas multiple quantum wells," JOSA B, vol. 11, no. 12, pp. 2502-2507, 1994.

[23] Z. Jiang and X.-C. Zhang, "Electro-optic measurement of thz field pulses with a chirped optical beam," Applied Physics Letters, vol. 72, no. 16, pp. 1945-1947, 1998.

[24] N. Matlis, G. Plateau, J. van Tilborg, and W. Leemans, "Singleshot spatiotemporal measurements of ultrashort thz waveforms using temporal electric-field cross correlation," JOSA B, vol. 28, no. 1, pp. 23-27, 2011. 
[25] K. Kim, B. Yellampalle, A. Taylor, G. Rodriguez, and J. Glownia, "Single-shot terahertz pulse characterization via two-dimensional electro-optic imaging with dual echelons," Optics letters, vol. 32, no. 14, pp. 1968-1970, 2007.

[26] A. Spiro and M. Lowe, "Method of ultrafast beam rotation for singleshot, time-resolved measurements," Optics letters, vol. 39, no. 18, pp. 5362-5365, 2014.

[27] P. Lane, P. Cunningham, J. Melinger, O. Esenturk, and E. Heilweil, "Hot photocarrier dynamics in organic solar cells," Nature communications, vol. 6, p. 7558, 2015.

[28] P. D. Cunningham and L. M. Hayden, "Carrier dynamics resulting from above and below gap excitation of p3ht and p3ht/pcbm investigated by optical-pump terahertz-probe spectroscopy," The Journal of Physical Chemistry C, vol. 112, no. 21, pp. 7928-7935, 2008.

[29] Z. Jin, D. Gehrig, C. Dyer-Smith, E. J. Heilweil, F. Laquai, M. Bonn, and D. Turchinovich, "Ultrafast terahertz photoconductivity of photovoltaic polymer-fullerene blends: A comparative study correlated with photovoltaic device performance," The journal of physical chemistry letters, vol. 5, no. 21, pp. 3662-3668, 2014.

[30] A. Nahata, A. S. Weling, and T. F. Heinz, "A wideband coherent terahertz spectroscopy system using optical rectification and electro-optic sampling," Applied physics letters, vol. 69, no. 16, pp. 2321-2323, 1996.

[31] Q. Wu and X.-C. Zhang, "Free-space electro-optics sampling of midinfrared pulses," Applied physics letters, vol. 71, no. 10, pp. 1285-1286, 1997.

[32] R. Kaindl, F. Eickemeyer, M. Woerner, and T. Elsaesser, "Broadband phase-matched difference frequency mixing of femtosecond pulses 
in gase: Experiment and theory," Applied Physics Letters, vol. 75, no. 8, pp. 1060-1062, 1999.

[33] R. Huber, A. Brodschelm, F. Tauser, and A. Leitenstorfer, "Generation and field-resolved detection of femtosecond electromagnetic pulses tunable up to 41 thz," Applied Physics Letters, vol. 76, no. 22, pp. 31913193, 2000.

[34] Q. Wu and X.-C. Zhang, "Free-space electro-optic sampling of terahertz beams," Applied Physics Letters, vol. 67, no. 24, pp. 3523-3525, 1995.

[35] A. Nahata, D. H. Auston, T. F. Heinz, and C. Wu, “Coherent detection of freely propagating terahertz radiation by electro-optic sampling," Applied physics letters, vol. 68, no. 2, pp. 150-152, 1996.

[36] R. T. Hall and J. M. Dowling, "Pure rotational spectrum of water vapor," The Journal of Chemical Physics, vol. 47, no. 7, pp. 2454-2461, 1967.

[37] D. E. Burch, "Absorption of infrared radiant energy by $\mathrm{CO}_{2}$ and $\mathrm{h}_{2} \mathrm{O}$. iii. absorption by $\mathrm{h}_{2} \mathrm{o}$ between 0.5 and $36 \mathrm{~cm}^{-1}(278 \mu-2 \mathrm{~cm})$, , JOSA, vol. 58, no. 10, pp. 1383-1394, 1968.

[38] A. Danylov, "Thz laboratory measurements of atmospheric absorption between $6 \%$ and $52 \%$ relative humidity," Submillimeter-Wave Technology Laboratory University of Massachusetts Lowell, vol. 175, 2006.

[39] J. Xu and X.-C. Zhang, "Optical rectification in an area with a diameter comparable to or smaller than the center wavelength of terahertz radiation," Optics letters, vol. 27, no. 12, pp. 1067-1069, 2002.

[40] P. C. Planken, H.-K. Nienhuys, H. J. Bakker, and T. Wenckebach, "Measurement and calculation of the orientation dependence of terahertz pulse detection in znte," JOSA B, vol. 18, no. 3, pp. 313-317, 2001. 
[41] J. Messer, F. C. De Lucia, and P. Helminger, "The pure rotational spectrum of water vapora millimeter, submillimeter, and far infrared analysis," International journal of infrared and millimeter waves, vol. 4, no. 4, pp. 505-539, 1983.

[42] Y. Minami, H. Yamaki, I. Katayama, and J. Takeda, "Broadband pump-probe imaging spectroscopy applicable to ultrafast single-shot events," Applied Physics Express, vol. 7, no. 2, p. 022402, 2014.

[43] T. C. Wong and R. Trebino, "Single-frame measurement of complex laser pulses tens of picoseconds long using pulse-front tilt in cross-correlation frequency-resolved optical gating," JOSA B, vol. 30, no. 11, pp. 2781-2786, 2013.

[44] T. C. Wong, M. Rhodes, and R. Trebino, "Single-shot measurement of the complete temporal intensity and phase of supercontinuum," Optica, vol. 1, no. 2, pp. 119-124, 2014.

[45] T. Zhang, Z. Jin, S. Wakamatsu, T. Hosokai, N. Yugami, and R. Kodama, "Note: Single-shot time-domain spectroscopy and spatial profiling of terahertz pulses from intense laser systems," Review of Scientific Instruments, vol. 87, no. 6, p. 066101, 2016.

[46] J. Shan, A. S. Weling, E. Knoesel, L. Bartels, M. Bonn, A. Nahata, G. A. Reider, and T. F. Heinz, "Single-shot measurement of terahertz electromagnetic pulses by use of electro-optic sampling," Optics letters, vol. 25 , no. 6 , pp. 426-428, 2000.

[47] Y. T. Mazurenko, "Holography of wave packets," Applied Physics B, vol. 50, no. 2, pp. 101-114, 1990.

[48] O. E. Martinez, "Pulse distortions in tilted pulse schemes for ultrashort pulses," Optics communications, vol. 59, no. 3, pp. 229-232, 1986.

[49] S. A. Akhmanov, V. A. Vysloukh, and A. S. Chirkin, "Optics of femtosecond laser pulses," Moscow Izdatel Nauka, 1988. 
[50] P. D. Cunningham, K. M. McCreary, A. T. Hanbicki, M. Currie, B. T. Jonker, and L. M. Hayden, "Charge trapping and exciton dynamics in large-area cvd grown mos2," The Journal of Physical Chemistry C, vol. 120, no. 10, pp. 5819-5826, 2016.

[51] F. A. Hegmann, O. Ostroverkhova, and D. G. Cooke, "Probing organic semiconductors with terahertz pulses," Photophysics of Molecular Materials: From Single Molecules to Single Crystals, pp. 367-428, 2006.

[52] P. D. Cunningham, N. N. Valdes, F. A. Vallejo, L. M. Hayden, B. Polishak, X.-H. Zhou, J. Luo, A. K.-Y. Jen, J. C. Williams, and R. J. Twieg, "Broadband terahertz characterization of the refractive index and absorption of some important polymeric and organic electro-optic materials," Journal of Applied Physics, vol. 109, no. 4, pp. 043505-043505, 2011.

[53] P. D. Cunningham, L. M. Hayden, H.-L. Yip, and A. K.-Y. Jen, “Charge carrier dynamics in metalated polymers investigated by opticalpump terahertz-probe spectroscopy," The Journal of Physical Chemistry B, vol. 113, no. 47, pp. 15427-15432, 2009.

[54] C. Ponseca Jr and V. Sundström, "Revealing the ultrafast charge carrier dynamics in organo metal halide perovskite solar cell materials using time resolved thz spectroscopy," Nanoscale, vol. 8, no. 12, pp. 6249-6257, 2016.

[55] C. S. Ponseca Jr, T. J. Savenije, M. Abdellah, K. Zheng, A. Yartsev, T. Pascher, T. Harlang, P. Chabera, T. Pullerits, A. Stepanov, et al., "Organometal halide perovskite solar cell materials rationalized: ultrafast charge generation, high and microsecond-long balanced mobilities, and slow recombination," Journal of the American Chemical Society, vol. 136, no. 14, pp. 5189-5192, 2014. 
[56] G. R. Yettapu, D. Talukdar, S. Sarkar, A. Swarnkar, A. Nag, P. Ghosh, and P. Mandal, "Terahertz conductivity within colloidal cspbbr3 perovskite nanocrystals: remarkably high carrier mobilities and large diffusion lengths," Nano letters, vol. 16, no. 8, pp. 4838-4848, 2016.

[57] K. Chen, Ultrafast Broadband Time-Resolved Photoluminescence Spectroscopy for Advanced Molecular Optoelectronic Materials. PhD thesis, Victoria University of Wellington, 2015.

[58] S. Nashima, O. Morikawa, K. Takata, and M. Hangyo, "Measurement of optical properties of highly doped silicon by terahertz time domain reflection spectroscopy," Applied physics letters, vol. 79, no. 24, pp. 3923-3925, 2001.

[59] J. Dai, J. Zhang, W. Zhang, and D. Grischkowsky, “Terahertz timedomain spectroscopy characterization of the far-infrared absorption and index of refraction of high-resistivity, float-zone silicon," JOSA B, vol. 21, no. 7, pp. 1379-1386, 2004.

[60] J. Li and J. Li, "Dielectric properties of silicon in terahertz wave region," Microwave and optical technology letters, vol. 50, no. 5, pp. 11431146, 2008.

[61] R. H. Giles, "Characterization of material properties at terahertz frequencies," tech. rep., MASSACHUSETTS UNIV LOWELL SUBMILLIMETER-WAVE TECH LAB, 1995.

[62] M. S. Tyagi and R. Van Overstraeten, "Minority carrier recombination in heavily-doped silicon," Solid-State Electronics, vol. 26, no. 6, pp. 577-597, 1983.

[63] C. Brückner, G. Notni, and A. Tünnermann, "Optimal arrangement of 90 off-axis parabolic mirrors in thz setups," Optik-International Journal for Light and Electron Optics, vol. 121, no. 1, pp. 113-119, 2010. 
Bibliography

[64] B. Hu and M. Nuss, "Imaging with terahertz waves," Optics letters, vol. 20, no. 16, pp. 1716-1718, 1995. 